Supporting Information (SI)

\title{
Pauli Paramagnetism of Stable Analogues of Pernigraniline Salt Featuring Ladder-Type Constitution
}

Xiaozhou Ji, , Haomiao Xie, , Congzhi Zhu, I Yang Zou, , Anthony U. Mu, , Mohammed Al-

Hashimi, 3 Kim R. Dunbar, 1 and Lei Fang ${ }_{1}^{*}$

I Department of Chemistry, Texas A\&M University, College Station, Texas 77843-3255, United States

2Shenzhen Key Laboratory of Polymer Science and Technology, College of Materials Science and Engineering, Shenzhen University, Shenzhen 518060, China

3 Department of Chemistry, Texas A\&M University at Qatar, P.O. Box 23874, Doha, Qatar

\section{Supporting Information}

\begin{tabular}{|c|}
\hline *Correspondence Address \\
\hline Professor Lei Fang \\
Department of Chemistry, Texas A\&M University, 3255 TAMU \\
College Station, TX 77845-3255, USA \\
Tel: +1 (979) 845 3186 \\
E-Mail: fang@ @ehem.tamu.edu \\
\hline
\end{tabular}




\section{Table of Content}

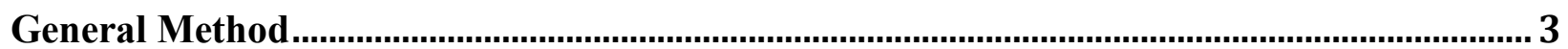

Condition Optimization of the Amine-Aryl Bromide Coupling Reaction ............................. 4

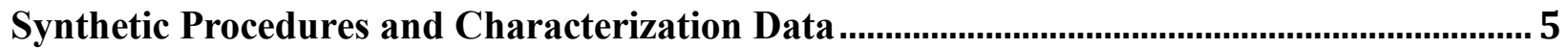

COSY NMR Spectroscopy of NLQ in Solution.....................................................................14

Determination of Energy Levels of Frontier Molecular Orbitals by Electrochemical

Analysis and Density Functional Theory Calculation ............................................................16

UV-vis-NIR Absorption Spectra Before and After Protonation.............................................18

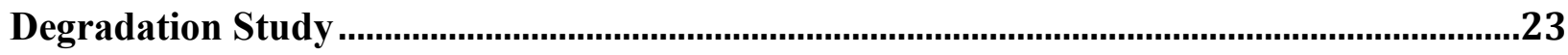

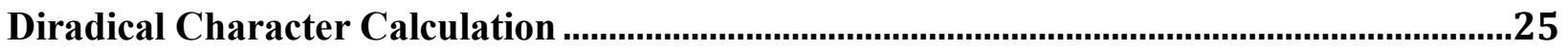

Electron Paramagnetic Resonance Spectroscopy …................................................................27

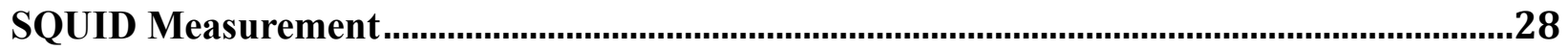

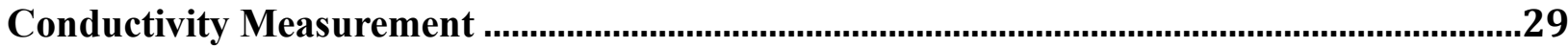

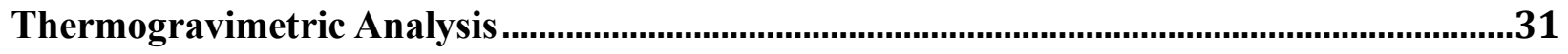

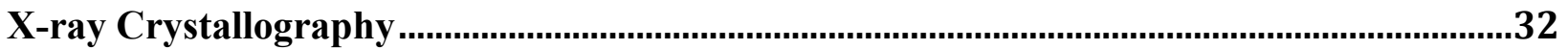

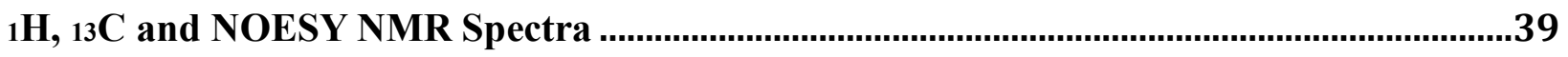




\section{General Method}

All reaction solvents were dried and purified by an Inert Technology pure solvent system (PureSolv-MD-5a). All starting materials were obtained from commercial suppliers and were used without further purification unless otherwise specified. $1 \mathrm{H}, 13 \mathrm{C}, 1 \mathrm{H}-1 \mathrm{H}$ COSY and ${ }_{1} \mathrm{H}-1 \mathrm{H}$ NOESY nuclear magnetic resonance (NMR) spectra were recorded on a Varian Inova $500 \mathrm{MHz}$ spectrometer at room temperature and processed by MestReNova 10.0.2. Chemical shifts are reported in ppm relative to the signals corresponding to the residual non-deuterated solvents (for 1H NMR: $\mathrm{CDCl}_{3} \delta=7.26 \mathrm{ppm} ; 1,1,2,2-\mathrm{C}_{2} \mathrm{D}_{2} \mathrm{Cl}_{4} \delta=6.00 \mathrm{ppm} ; \mathrm{CD}_{2} \mathrm{Cl}_{2} \delta=5.32 \mathrm{ppm}$; for ${ }_{13} \mathrm{C} \mathrm{NMR}:$ $\mathrm{CDCl}_{3} \delta=77.16 \mathrm{ppm}$ ). Flash column chromatography purifications were carried out using a Biotage ${ }^{\circledR}$ Isolera ${ }^{\mathrm{TM}}$ Prime with various sizes of $\mathrm{SiO}_{2}$ Biotage SNAP ${ }^{\circledR}$ cartridges. Preparative Size Exclusive Chromatography (SEC) purifications were performed at room temperature using a JAI recycling preparative HPLC (LC-92XXII NEXT SERIES) equipped with a reciprocating double plunger pump (model P-9104B), a UV-vis 4ch NEXT detector (254 nm, $280 \mathrm{~nm}, 300 \mathrm{~nm}, 3300$ $\mathrm{nm}$ ), and a two-column set including a JAIGEL-H 40P Gard column and a JAIGEL-2H-40 HPLC column. Chloroform as the eluent at a flow rate of $14 \mathrm{~mL} / \mathrm{min}$. UV-Vis-NIR absorption spectra were recorded on a Hitachi U-4100 UV-Vis-NIR spectrophotometer. Electrospray ionization mass spectrometry (ESI-MS) experiments were performed using a Thermo Scientific Q-Exactive Focus operated in full MS in positive mode. High resolution Matrix-assisted laser desorption ionizationtime of flight mass spectrometry (MALDI-TOF MS) was performed on a microflex ${ }^{\mathrm{TM}}$ LRF mass spectrometer (Bruker Corporation) in positive linear mode with 2,4,6-trihydroxyacetophenone monohydrate (THAP) as the matrix. 


\section{Condition Optimization of the Amine-Aryl Bromide Coupling Reaction}

Condition screening was conducted on the Buchwald-Hartwig cross-coupling reactions between diamino fluorene $\mathbf{4}$ and ester functionalized aryl dibromide B1, by varying the molar ratio between starting materials, catalyst and ligand loading, and temperature. The optimized condition is highlighted in bold in Table S1.

Table S1. Screened Conditions and Results of Buchwald-Hartwig Reaction for Synthesis of B2

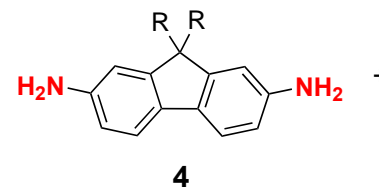

4

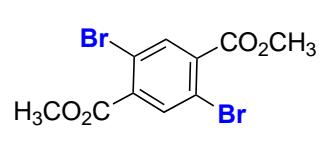

B1

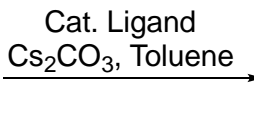

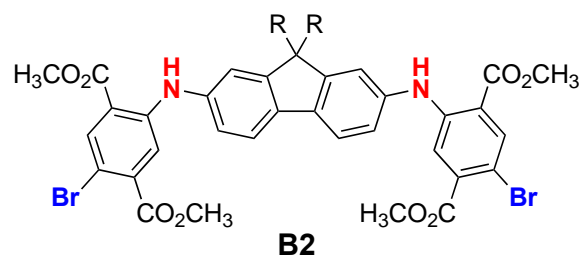

\section{Equiv. (4 / B1)}

Catalyst (mol \%)

Ligand (mol \%)

$\mathbf{T}\left({ }^{\circ} \mathbf{C}\right)$

Yield (\%)a

$1 / 3.0$

$\mathrm{Pd}_{2}(\mathrm{dba}) 3(10 \%)$

$t \mathrm{Bu} 3 \mathrm{P}(40 \%)$

120

Xphos (40 \%)

120

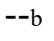

$1 / 3.0$

$\mathrm{Pd}_{2}(\mathrm{dba})_{3}(10 \%)$

BINAP (30 \%)

120

55

$1 / 3.0$

$\operatorname{Pd}(\mathrm{OAc}) 2(20 \%)$

BINAP (30 \%)

120

44

$1 / 3.5$

$\mathrm{Pd}(\mathrm{OAc})_{2}(20 \%)$

BINAP (15\%)

110

--b

$1 / 3.5$

$\mathrm{Pd}_{2}(\mathrm{dba})_{3}(10 \%)$

BINAP (30 \%)

110

28

$1 / 3.0$

$\operatorname{Pd}(\mathrm{OAc}) 2(10 \%)$

Xantphos (15\%)

110

56

$1 / 4.0$

$\operatorname{Pd}(\mathrm{OAc}) 2(10 \%)$

Xantphos (15\%)

120

87

aIsolated yield; bOligomeric by-products were observed. 


\section{Synthetic Procedures and Characterization Data}

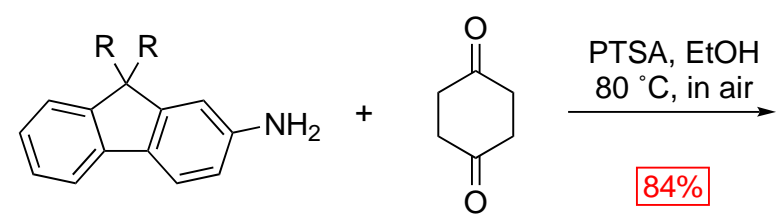

$\mathrm{R}=n$-hexyl

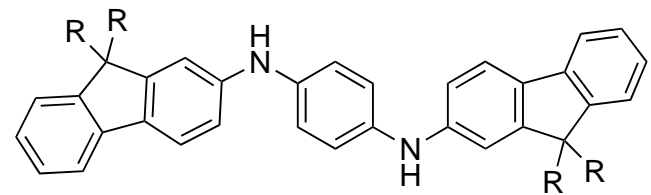

NLA

Scheme S1. Synthesis of NLA via imine condensation.

NLA: 9,9-dihexyl-2-amino fluorene ( $0.51 \mathrm{~g}, 1.46 \mathrm{mmol}), 1,4$-cyclohexanone $(0.065 \mathrm{~g}, 0.58 \mathrm{mmol})$, p-toluenesulfonic acid monohydrate $(22 \mathrm{mg}, 0.12 \mathrm{mmol})$, and ethanol $(15 \mathrm{~mL})$ were charged in a $50 \mathrm{~mL}$-round-bottom flask with a condenser. The mixture was stirred open to the air at $80{ }^{\circ} \mathrm{C}$ for $12 \mathrm{~h}$. After cooled to room temperature, the precipitate was filtered and washed with methanol to give the product as a grey powder $(0.47 \mathrm{~g}, 84 \%)$. ${ }_{1} \mathrm{H}$ NMR $\left(500 \mathrm{MHz}, \mathrm{CD}_{2} \mathrm{Cl}_{2}, 298 \mathrm{~K}\right) \delta=7.63-$ $7.57(\mathrm{~m}, 4 \mathrm{H}), 7.34-7.28(\mathrm{~m}, 4 \mathrm{H}), 7.23\left(\mathrm{td}, J_{1}=7.4 \mathrm{~Hz}, J_{2}=1.1 \mathrm{~Hz}, 2 \mathrm{H}\right), 7.14(\mathrm{~s}, 4 \mathrm{H}), 7.05(\mathrm{~d}, J$ $=2.1 \mathrm{~Hz}, 2 \mathrm{H}), 7.00\left(\mathrm{dd}, J_{1}=8.2, J_{2}=2.2 \mathrm{~Hz}, 2 \mathrm{H}\right), 5.84(\mathrm{~s}, 2 \mathrm{H}), 2.01-1.88(\mathrm{~m}, 8 \mathrm{H}), 1.21-1.03$ (m, 24H), $0.80(\mathrm{t}, J=7.2 \mathrm{~Hz}, 12 \mathrm{H}), 0.76-0.59(\mathrm{~m}, 8 \mathrm{H}) .{ }_{13} \mathrm{C} \mathrm{NMR}(125 \mathrm{MHz}, \mathrm{cdcl} 3) \delta=152.62$ $150.21,143.65,141.44,137.62,134.14,126.78,125.79,122.79,120.56,120.38,118.72,115.82$ 111.72, 55.06, 40.67, 31.69, 29.90, 23.91, 22.76, 14.18. HRMS (+ESI): $\mathrm{C}_{56} \mathrm{H}_{72} \mathrm{~N}_{2}[\mathrm{M}+\mathrm{H}]+$ calcd. $m / z=773.5768$, found $m / z=773.5725$.
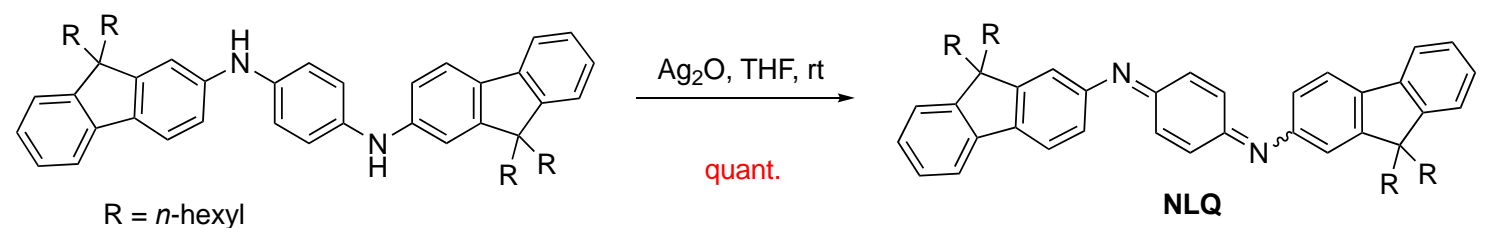

Scheme S2. Synthesis of NLQ via oxidation

NLQ: $\mathrm{Ag}_{2} \mathrm{O}(60 \mathrm{mg})$ was added into a solution of NLA $(0.1 \mathrm{~g}, 0.13 \mathrm{mmol})$ in THF $(10 \mathrm{~mL})$. The mixture was stirred at room temperature in ambient condition for $12 \mathrm{~h}$. The precipitate was filtered out and the filtrate was concentrated with a rotatory evaporator, affording the product a purple oil 
(0.1 g, quant.). ${ }_{1} \mathrm{H}$ NMR (500 MHz, $\left.\mathrm{CD}_{2} \mathrm{Cl}_{2}, 298 \mathrm{~K}\right) \delta=7.80-7.69(\mathrm{~m}, 4 \mathrm{H}), 7.43-7.30(\mathrm{~m}, 6 \mathrm{H})$, $7.19-7.15(\mathrm{~s}, 0.84 \mathrm{H}), 7.05-7.01(\mathrm{~s}, 2.32 \mathrm{H}), 6.98-6.92(\mathrm{~s}, 3.16 \mathrm{H}), 6.91-6.85(\mathrm{~m}, 1.68 \mathrm{H}), 2.01$ $(\mathrm{m}, 8 \mathrm{H}), 1.22-1.01(\mathrm{~m}, 27.36 \mathrm{H}), 0.82(\mathrm{t}, J=7.3 \mathrm{~Hz}, 5.04 \mathrm{H}), 0.79-0.58(\mathrm{~m}, 11.6 \mathrm{H}) .13 \mathrm{C} \mathrm{NMR}$ $\left(125 \mathrm{MHz}, \mathrm{CDCl}_{3}, 298 \mathrm{~K}\right) \delta=158.45,158.30,152.00,151.93,150.74,150.70,149.28,140.56$, $140.52,138.94,138.92,137.62,136.53,126.99,126.97,126.87,126.84,125.08,124.49,122.81$, $120.09,119.94,119.70,119.51,119.46,116.22,116.03,77.27,77.01,76.76,55.20,55.17,40.46$, $40.41,31.52,31.49,29.69,29.65,23.79,23.74,22.56,22.53,14.00,13.96$. HRMS (+ESI): $\mathrm{C}_{56} \mathrm{H}_{70} \mathrm{~N}_{2}[\mathrm{M}+\mathrm{H}]+$ calcd. $m / z=771.5612$, found $m / z=771.5550$.

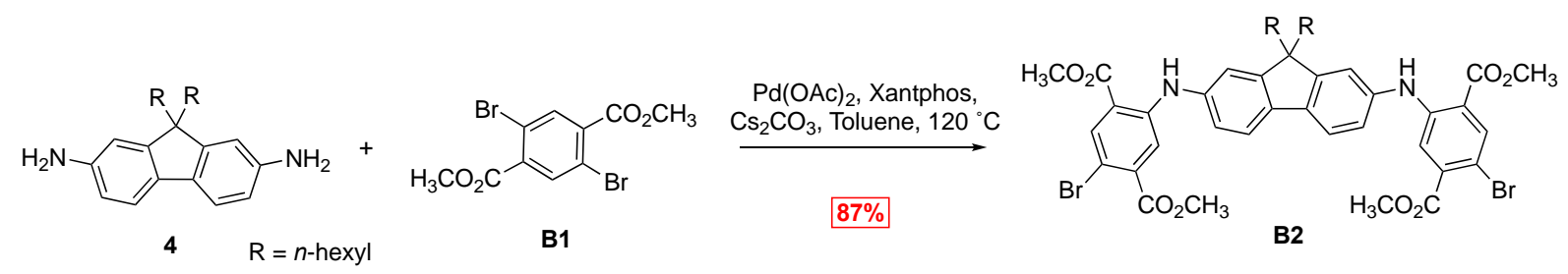

Scheme S3. Synthesis of B2 via Buchwald-Hartwig coupling reaction.

B2: 9,9'-dihexyl-2,7-diaminofluorene 4 (0.19 g, $0.54 \mathrm{mmol})$, dimethyl 2,5-dibromoterephthalate B1 (0.75 g, $2.14 \mathrm{mmol}), \mathrm{Pd}(\mathrm{OAc}) 2$ (12 mg, $0.054 \mathrm{mmol})$, Xantphos (47 mg, $0.081 \mathrm{mmol})$, and $\mathrm{Cs}_{2} \mathrm{CO}_{3}(1.05 \mathrm{~g}, 3.24 \mathrm{mmol})$ were mixed in $10 \mathrm{~mL}$ of dry toluene in a $50 \mathrm{~mL}-$ Schlenk tube. After freeze-pump-thaw for 3 times, the mixture was heated at $120{ }^{\circ} \mathrm{C}$ and stirred for $12 \mathrm{~h}$. After cooling down, the mixture was diluted in dichloromethane and filtered through a Celite pack and concentrated by the rotatory evaporator. It was further purified by flash column chromatography $\left(\mathrm{SiO}_{2}\right.$, hexane/ethyl acetate $\left.7: 1\right)$. The product was isolated as a yellow powder $(0.42 \mathrm{~g}, 87 \%) .{ }_{1} \mathrm{H}$ $\operatorname{NMR}\left(500 \mathrm{MHz}, \mathrm{CDCl}_{3}, 298 \mathrm{~K}\right): \delta=0.68\left(\mathrm{qd}, 4 \mathrm{H}, J_{1}=3.2 \mathrm{~Hz}, J_{2}=2.5 \mathrm{~Hz}, 4 \mathrm{H}\right), 0.77(\mathrm{t}, J=7.1$ Hz, 6H), 1.07-1.16 (m, 12H), $1.92(\mathrm{~m}, 4 \mathrm{H}), 3.86(\mathrm{~s}, 6 \mathrm{H}), 3.95(\mathrm{~s}, 6 \mathrm{H}), 7.16(\mathrm{~d}, J=1.9 \mathrm{~Hz}, 2 \mathrm{H}$,$) ,$ $7.20(\mathrm{dd}, J=8.0 \mathrm{~Hz}, 2 \mathrm{H}), 7.64(\mathrm{~d}, J=8.0 \mathrm{~Hz}, 2 \mathrm{H}), 8.21(\mathrm{~s}, 2 \mathrm{H}), 9.54(\mathrm{~s}, 2 \mathrm{H}) .13 \mathrm{C} \mathrm{NMR}(125 \mathrm{~Hz}$, $\left.\mathrm{CDCl}_{3}, 298 \mathrm{~K}\right): \delta=14.2,22.9,24.1,30.0,31.8,40.7,52.5,52.8,55.5,105.9,114.6,116.5,117.8$ 
$120.5,122.0,136.7,137.5,137.6,138.5,147.2,152.6,166.6,167.6$. HRMS (+ESI): $\mathrm{C}_{45} \mathrm{H}_{50} \mathrm{Br}_{2} \mathrm{~N}_{2} \mathrm{O} 8[\mathrm{M}+\mathrm{H}]+$ calcd. $m / z=907.1987$, found $m / z=907.1954$.

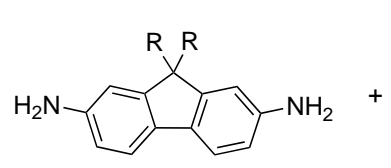

4

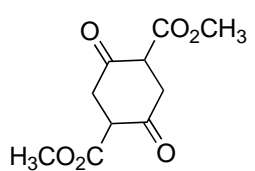

2

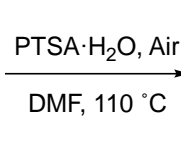

$74 \%$

Scheme S4. Synthesis of 6 via imine-condensation.

Compound 6: 2,7-diamino-9,9-hexyl-fluorene 4 (0.65 g, $1.78 \mathrm{mmol})$, dimethyl succinylsuccinate $2(0.16 \mathrm{~g}, 0.70 \mathrm{mmol})$, and $p$-toluenesulfonic acid $(30 \mathrm{mg}, 0.14 \mathrm{mmol})$ was dissolved in DMF (10 $\mathrm{mL}$ ). The mixture was heat to $110{ }^{\circ} \mathrm{C}$ under $\mathrm{N}_{2}$ for $4 \mathrm{~h}$ and exposed to air for $8 \mathrm{~h}$. After cooling down, the mixture was poured into water and extracted with ethyl acetate ( $10 \mathrm{~mL}$ for 3 times). The combined organic layer was washed with water and brine. After removing the solvent, the crude product was purified by flash column chromatography ( $\mathrm{SiO}_{2}$, hexane/ethyl acetate 6:1). The pure product was isolated as a dark red powder $(0.60 \mathrm{~g}, 74 \%)$. $1 \mathrm{H} \mathrm{NMR}\left(500 \mathrm{MHz}, \mathrm{CDCl}_{3}, 298 \mathrm{~K}\right): \delta=$ $0.72(\mathrm{~m}, 8 \mathrm{H}), 0.75-0.85$ (t, 12H), 0.97-1.19 (m, 24H), 1.87 (t, 8H, $J=8.3 \mathrm{~Hz}), 3.73$ (br, 4H), 3.85 (s, 6H), 6.61-6.71 (m, 4H), 7.06-7.14 (m, 4H), $7.42(\mathrm{~d}, 2 \mathrm{H}, J=8.3 \mathrm{~Hz}), 7.50(\mathrm{~d}, 2 \mathrm{H}, J=8.0 \mathrm{~Hz})$ $8.01(\mathrm{~s}, 2 \mathrm{H}), 8.86(\mathrm{~s}, 2 \mathrm{H}) 13 \mathrm{C} \mathrm{NMR}\left(126 \mathrm{~Hz}, \mathrm{CDCl}_{3}, 298 \mathrm{~K}\right): \delta=14.0,22.7,23.8,29.9,31.6,40.8$ $52.2,54.8,110.0,113.9,115.0,117.9,118.3,119.0,119.7,132.5,136.3,138.2,139.6,145.2,151.4$, 152.2, 168.2. HRMS (+ESI): $\mathrm{C}_{60} \mathrm{H}_{78} \mathrm{~N}_{4} \mathrm{O}_{4}[\mathrm{M}+\mathrm{H}]+$ calcd. $m / z=919.6096$, found $m / z=919.6071$.

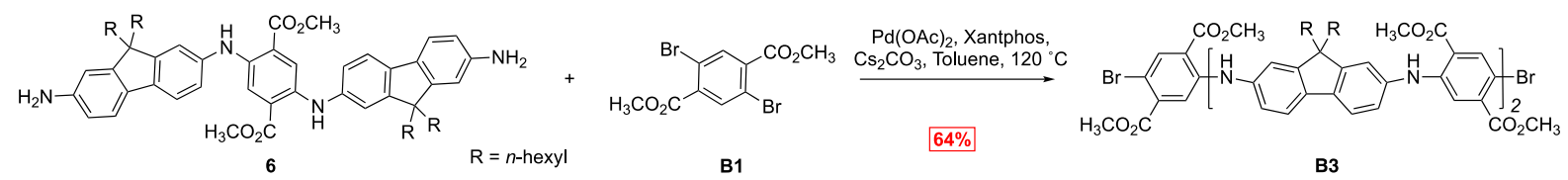

Scheme S5. Synthesis of B3 via Buchwald-Hartwig coupling reaction

B3: Compound 6 (0.12 g, $0.13 \mathrm{mmol})$, dimethyl 2,5-dibromoterephthalate B1 (0.18 g, $0.52 \mathrm{mmol})$, $\mathrm{Pd}(\mathrm{OAc}) 2(3 \mathrm{mg}, 0.013 \mathrm{mmol})$, Xantphos (12 mg, $0.019 \mathrm{mmol})$, and $\mathrm{Cs}_{2} \mathrm{CO}_{3}(0.17 \mathrm{~g}, 0.52 \mathrm{mmol})$ 
were mixed in $5 \mathrm{~mL}$ of anhydrous toluene in a $50 \mathrm{~mL}$ Schlenk tube. After freeze-pump-thaw for 3 times, the reaction was heated to $110{ }^{\circ} \mathrm{C}$ under nitrogen protection and stirred for $12 \mathrm{~h}$. After cooling down, the mixture was diluted with dichloromethane and filtered through a Celite pack and concentrated by a rotatory evaporator. It was further purified by flash column chromatography $\left(\mathrm{SiO}_{2}\right.$, hexane/ethyl acetate 20:1 to 5:1). The pure product was isolated as a red powder $(0.19 \mathrm{~g}$, 64\%). $1 \mathrm{H}$ NMR (500 MHz, $\left.\mathrm{CDCl}_{3}, 298 \mathrm{~K}\right): \delta=0.77(\mathrm{~m}, 20 \mathrm{H}), 1.12(\mathrm{~m}, 32 \mathrm{H}), 1.92(\mathrm{t}, 8 \mathrm{H}, J=8.4$ $\mathrm{Hz}), 3.86(\mathrm{~s}, 6 \mathrm{H}), 3.88(\mathrm{~s}, 6 \mathrm{H}), 3.96(\mathrm{~s}, 6 \mathrm{H}), 7.17(\mathrm{~m}, 8 \mathrm{H}), 7.56(\mathrm{~s}, 2 \mathrm{H}), 7.61\left(\mathrm{dd}, 4 \mathrm{H}, J_{1}=7.9, J_{2}=\right.$ $3.3 \mathrm{~Hz}), 8.09$ (s, 2H), 8.21 (s, 2H), 8.97 (s, 2H), 9.52 (s, 2H). ${ }_{13} \mathrm{C} \mathrm{NMR}\left(126 \mathrm{~Hz}, \mathrm{CDCl}_{3}, 298 \mathrm{~K}\right): \delta$ $=14.1,22.7,23.8,24.0,24.7,29.9,30.0,31.6,36.6,40.6,40.7,52.3,52.7,54.9,55.2,105.4,114.2$, $116.2,116.2,117.9,118.3,118.6,118.7,119.8,120.2,121.9,122.0,135.1,136.4,136.5,137.6$ 137.9, 138.1, 140.9, 147.3, 152.1, 152.3, 166.5, 167.5, 168.1. HRMS (+ESI): $\mathrm{C}_{80} \mathrm{H}_{92} \mathrm{Br}_{2} \mathrm{~N}_{4} \mathrm{O}_{12}$ $[\mathrm{M}+\mathrm{H}]+$ calcd. $m / z=1461.5153$, found $m / z=1461.5086$.
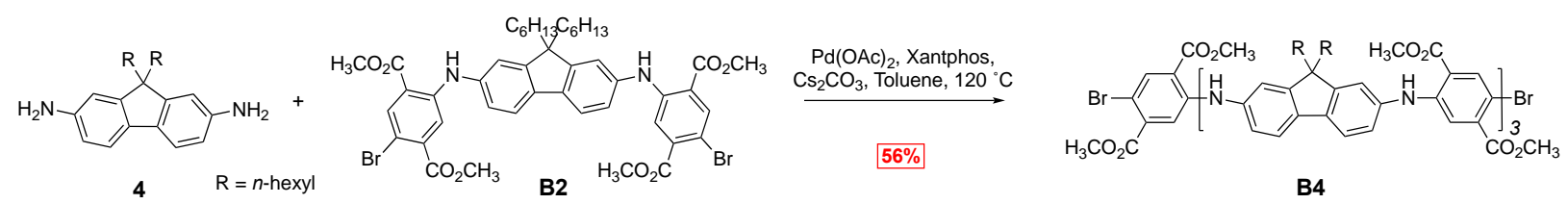

Scheme S6. Synthesis of B4 via Buchwald-Hartwig coupling reaction

B4: 9,9-dihexyl-2,7-diaminofluorene 4 (16 mg, $0.044 \mathrm{mmol})$, B2 (0.16 g, $0.18 \mathrm{mmol}), \mathrm{Pd}(\mathrm{OAc}) 2$ (2.4 mg,0.01 mmol), Xantphos (10 mg, $0.015 \mathrm{mmol}$ ), and $\mathrm{Cs}_{2} \mathrm{CO}_{3}(0.14 \mathrm{~g}, 0.42 \mathrm{mmol})$ were mixed in $5 \mathrm{~mL}$ of dry toluene in a $50 \mathrm{~mL}$ Schlenk tube. After freeze-pump-thaw for 3 times, the reaction was heated up to $130{ }^{\circ} \mathrm{C}$ under nitrogen and stirred for $12 \mathrm{~h}$. After cooling down, the mixture was diluted in dichloromethane and filtered through a Celite pack. Followed by being concentrated by a rotatory evaporator, the crude product was further purified by flash column chromatography ( $\mathrm{SiO}_{2}$, hexane/ethyl acetate $20: 1$ to $\left.7: 1\right)$. The pure product was isolated as a red powder $(80 \mathrm{mg}$, 56\%). $\left.1 \mathrm{H} \mathrm{NMR} \mathrm{(500} \mathrm{MHz,} \mathrm{CDCl}_{3}, 298 \mathrm{~K}\right): \delta=0.72(\mathrm{~m}, 12 \mathrm{H}), 0.78(\mathrm{~m}, 18 \mathrm{H}), 0.99-1.20(\mathrm{~m}, 36 \mathrm{H})$, 
$1.92(\mathrm{t}, 12 \mathrm{H}, J=8.4 \mathrm{~Hz}), 3.86(\mathrm{~s}, 6 \mathrm{H}), 3.88(\mathrm{~s}, 6 \mathrm{H}), 3.89(\mathrm{~s}, 6 \mathrm{H}), 3.96(\mathrm{~s}, 6 \mathrm{H}), 7.10-7.21(\mathrm{~m}, 12 \mathrm{H})$, $7.55(\mathrm{~s}, 2 \mathrm{H}), 7.57-7.61(\mathrm{~m}, 4 \mathrm{H}), 8.07(\mathrm{~s}, 2 \mathrm{H}), 8.09(\mathrm{~s}, 2 \mathrm{H}), 8.21(\mathrm{~s}, 2 \mathrm{H}), 8.94(\mathrm{~s}, 2 \mathrm{H}), 8.96(\mathrm{~s}, 2 \mathrm{H})$ 9.52 (s, 2H). ${ }_{13} \mathrm{C}$ NMR (126 MHz, $\left.\mathrm{CDCl}_{3}, 298 \mathrm{~K}\right) \delta=168.1,167.4,166.5,152.3,152.1,152.0$, 147.3, 141.0, 140.2, 138.3, 138.1, 137.6, 137.3, 136.5, 135.8, 134.9, 121.9, 120.2, 119.7, 119.0, $118.8,118.6,118.3,118.1,117.9,116.2,114.8,114.2,105.4,105.1,77.3,77.0,76.8,55.2,55.1$ 52.6, 52.3, 40.7, 40.6, 31.7, 31.6, 30.0, 29.9, 29.7, 23.94, 22.7, 22.7, 14.0, 14.0. HRMS (+ESI): $\mathrm{C}_{115} \mathrm{H}_{134} \mathrm{Br}_{2} \mathrm{~N}_{6} \mathrm{O}_{16}[\mathrm{M}+\mathrm{H}]+$ calcd. $m / z=2016.8236$, Found $m / z=2016.9336$.

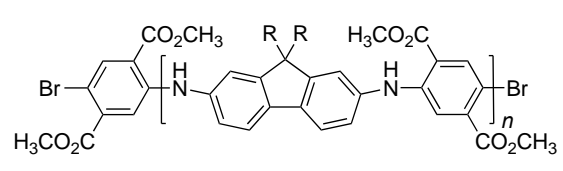

B2 $(n=1)$, B3 $(n=2)$, B4 $(n=3)$

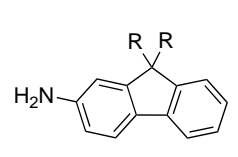

$\mathrm{R}=n$-hexyl

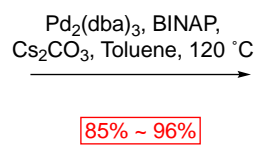

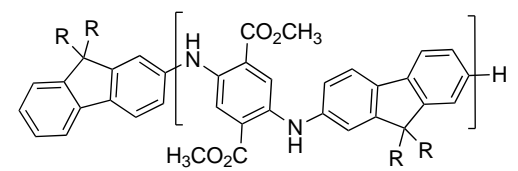

A2 $(n=1)$, A3 $(n=2)$, A4 $(n=3)$

Scheme S7. Synthesis of A2, A3, A4 via Buchwald-Hartwig coupling reaction

General Procedure to A2, A3, A4: Compound Bn (n=1, 2, 3) (ca. 0.1 mmol scale, 1 equiv.), 2amino-9,9-hexyl-fluorene (3 equiv.), $\mathrm{Pd}_{2}(\mathrm{dba})_{3}$ (10 mol\%), BINAP (30 mol\%), and $\mathrm{Cs}_{2} \mathrm{CO}_{3}$ (4 equiv.) mixed dissolved in $5 \mathrm{~mL}$ dry toluene in a Schlenk flask. After freeze-pump-thaw for 3 times, the reaction was heat to $120^{\circ} \mathrm{C}$ under nitrogen for $12 \mathrm{~h}$. After cooling down to room temperature, the mixture was diluted by dichloromethane and filtered through Celite. After removing the solvent, the crude product was recrystallized in methanol to give the pure product.

A2: $\operatorname{Red} \operatorname{solid}(0.15 \mathrm{~g}, 85 \%) .1 \mathrm{H} \mathrm{NMR}\left(500 \mathrm{MHz}, \mathrm{CDCl}_{3}, 298 \mathrm{~K}\right): \delta=0.78(\mathrm{~m}, 18 \mathrm{H}), 1.10(\mathrm{~m}, 48 \mathrm{H})$, $1.95(\mathrm{~m}, 12 \mathrm{H}), 3.88(\mathrm{~d}, 12 \mathrm{H}, J=1.1 \mathrm{~Hz}), 7.11-7.21(\mathrm{~m}, 8 \mathrm{H}), 7.31\left(\mathrm{ddd}, 4 \mathrm{H}, J_{1}=7.1, J_{2}=6.2, J_{3}=\right.$ 1.4Hz), 7.56-7.69 (m, 8H), $8.08\left(\mathrm{dd}, 4 \mathrm{H}, J_{1}=13.3, J_{2}=1.0 \mathrm{~Hz}\right), 8.94(\mathrm{~d}, 4 \mathrm{H}, J=10.5 \mathrm{~Hz}) .{ }_{13} \mathrm{C}$ $\operatorname{NMR}\left(125 \mathrm{~Hz}, \mathrm{CDCl}_{3}, 298 \mathrm{~K}\right): \delta=14.01,22.64,23.81,29.86,31.56,40.52,52.28,55.01,114.23$, $114.81,118.07,118.35,118.57,118.76,118.98,119.71,120.47,122.77,126.03,135.52,135.75$ 137.73, 138.27, 140.23, 141.13, 150.21, 152.02, 152.44, 168.10. HRMS (+ESI): $\mathrm{C}_{95} \mathrm{H}_{118 \mathrm{~N}} 4 \mathrm{O} 8$ $[\mathrm{M}+\mathrm{H}]+$ calcd. $m / z=1443.8983$, found $m / z=1443.8986$. 
A3: Red solid (0.14 g, 95\%). $1 \mathrm{H}$ NMR (500 MHz, CDCl $3,298 \mathrm{~K}): \delta=0.59-0.78(\mathrm{~m}, 40 \mathrm{H}), 0.99-$ $1.31(\mathrm{~m}, 48 \mathrm{H}), 1.94(\mathrm{t}, 16 \mathrm{H}, J=8.4 \mathrm{~Hz}), 3.88(\mathrm{~d}, 18 \mathrm{H}, J=2.9 \mathrm{~Hz}), 7.12-7.20(\mathrm{~m}, 12 \mathrm{H}), 7.29-7.34$ $(\mathrm{m}, 4 \mathrm{H}), 7.59(\mathrm{~d}, 4 \mathrm{H}, J=8.0 \mathrm{~Hz}), 7.64(\mathrm{~m}, 4 \mathrm{H}), 8.07(\mathrm{~d}, 4 \mathrm{H}, J=3.8 \mathrm{~Hz}), 8.09(\mathrm{~s}, 2 \mathrm{H}), 8.95(\mathrm{~d}, 6 \mathrm{H}$, $J=10.2 \mathrm{~Hz}) .13 \mathrm{C} \mathrm{NMR}\left(126 \mathrm{MHz}, \mathrm{CDCl}_{3}, 298 \mathrm{~K}\right) \delta=168.3,168.2,152.6,152.2,150.4,141.3$, $141.2,140.47,140.39,138.46,138.25,137.90,135.94,135.87,135.70,126.86,126.19,122.93$ $120.62,119.86,119.18,119.10,119.01,118.94,118.76,118.67,118.51,118.31,118.23,115.01$, $114.93,114.42,77.40,77.15,76.89,55.23,55.17,52.41,40.81,40.66,31.80,31.71,30.15,30.00$ 29.85, 24.09, 23.97, 22.86, 22.78, 14.16, 14.14. HRMS (+ESI): $\mathrm{C}_{130} \mathrm{H}_{160} \mathrm{~N}_{6} \mathrm{O} 12[\mathrm{M}+\mathrm{H}]+$ calcd. $m / z$ $=1999.2201$, Found $\mathrm{m} / \mathrm{z}=1999.2889$.

A4: Red solid (0.12 g, 96\%). $1 \mathrm{H}$ NMR (500 MHz, $\left.\mathrm{CDCl}_{3}, 298 \mathrm{~K}\right): \delta=0.60-0.83(\mathrm{~m}, 50 \mathrm{H}), 0.95-$ $1.22(\mathrm{~m}, 60 \mathrm{H}), 1.81-2.03(\mathrm{~m}, 40 \mathrm{H}), 3.81-3.91(\mathrm{~m}, 24 \mathrm{H}), 7.12-7.20(\mathrm{~m}, 16 \mathrm{H}), 7.31(\mathrm{~m}, 6 \mathrm{H}), 7.58-$ $7.66(\mathrm{~m}, 10 \mathrm{H}), 8.00-8.11(\mathrm{~m}, 8 \mathrm{H}), 8.86-8.99(\mathrm{~m}, 8 \mathrm{H}) .{ }_{13} \mathrm{C} \mathrm{NMR}\left(125 \mathrm{MHz}, \mathrm{CDCl}_{3}, 298 \mathrm{~K}\right) \delta=$ $168.1,168.1,152.5,152.0,150.2,141.1,141.1,140.3,140.2,138.3,138.1,137.7,135.8,135.7$, 135.5, 126.7, 126.0, 122.8, 120.5, 119.7, 119.0, 118.9, 118.8, 118.6, 118.5, 118.4, 118.1, 114.8, 114.2, 55.1, 55.0, 52.3, 40.7, 40.5, 31.7, 31.6, 31.6, 30.0, 29.9, 24.0, 23.8, 22.7, 22.7, 14.0, 14.0. HRMS (+MALDI-TOF): $\mathrm{C}_{165} \mathrm{H}_{202} \mathrm{~N}_{8} \mathrm{O} 16[\mathrm{M}+\mathrm{H}]+$ calcd. $m / z=2552.5212$, found $m / z=2552.5985$. 

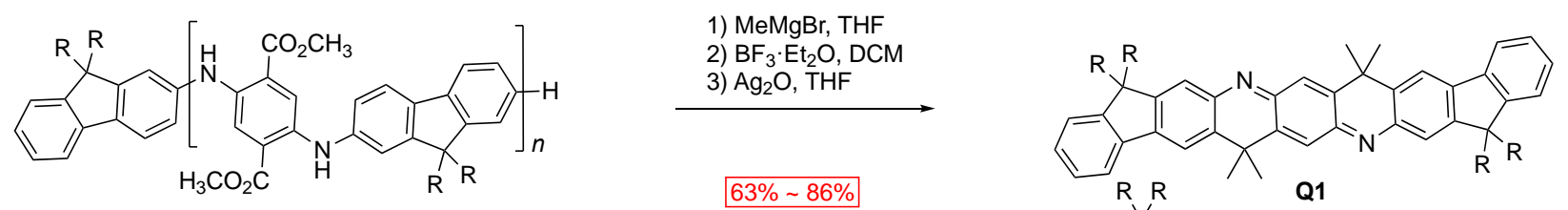

A1 $(n=0)$, A2 $(n=1)$, A3 $(n=2)$, A4 $(n=3)$

$\mathrm{R}=n$-hexyl
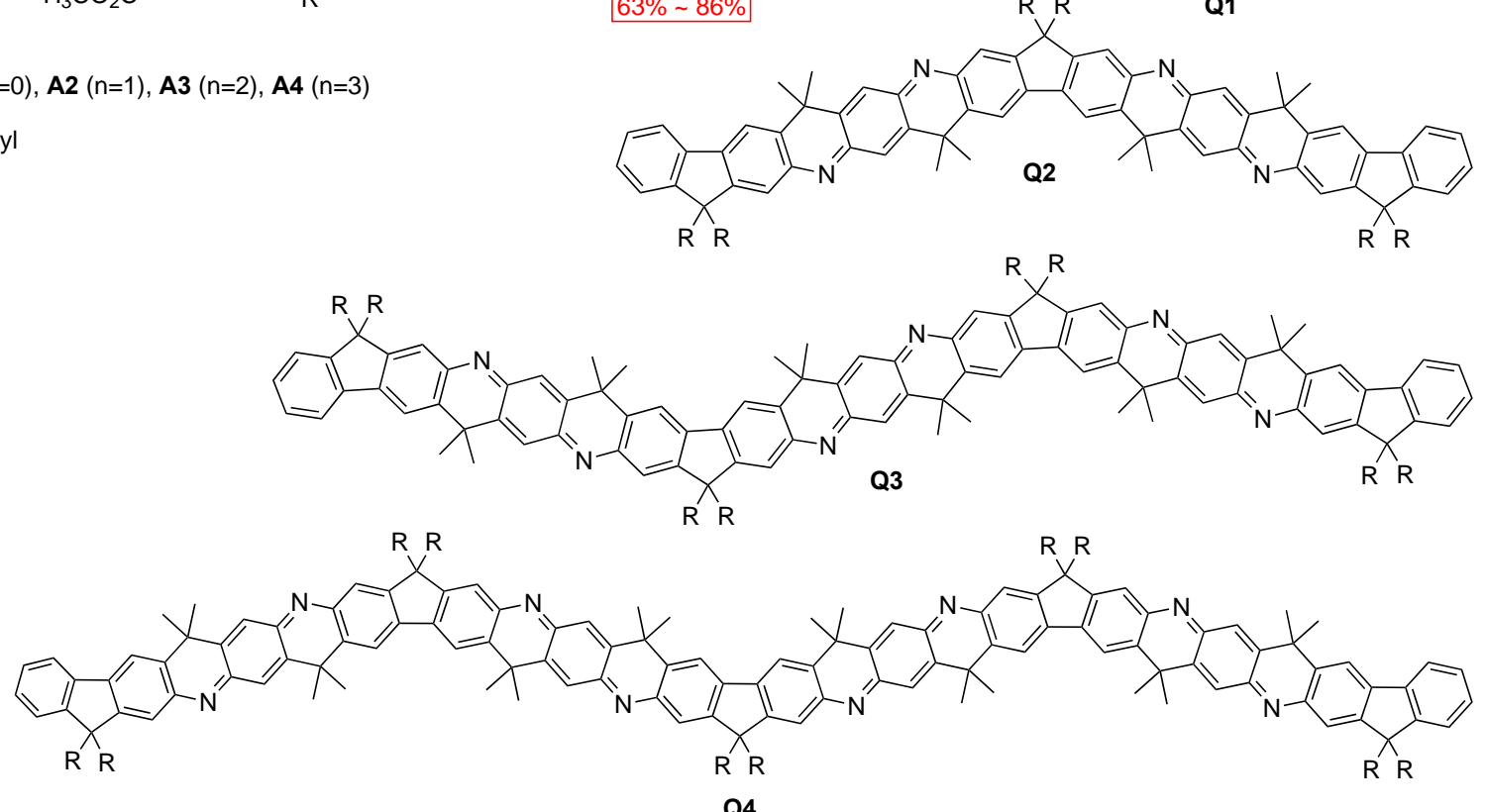

Scheme S8. Synthesis of Q1, Q2, Q3, and Q4.

General Procedure to Q1, Q2, Q3, and Q4: To a solution of methylmagnesium bromide (3M in diethyl ester) in dry THF (10n equiv.), a THF solution of $\mathbf{A n}(\mathrm{n}=1,2,3,4)$ (ca. $0.1 \mathrm{mmol}, 1$ equiv.) was added dropwise at $0{ }^{\circ} \mathrm{C}$ in $30 \mathrm{~min}$. The reaction mixture was heat up to $35^{\circ} \mathrm{C}$ for $12 \mathrm{~h}$. After cooled to room temperature, the reaction was quenched with saturated aqueous ammonia chloride solution. Ethyl acetate was used to extract the mixture for 3 times. The combined organic layer was washed with brine. After dried with anhydrous sodium sulfonate, the solvent was removed and the residue was re-dissolved in dry dichloromethane. Boron trifluoride diethyl etherate $(1 \mathrm{M})$ ( 1 equiv.) was added into the solution. The reaction was run under nitrogen protection at room temperature for $12 \mathrm{~h}$ and quenched with sodium hydroxide aqueous solution $(1 \mathrm{M})$. After extraction with ethyl acetate, the combined organic layer was washed with brine. After removing the solvent, the crude product was directly used in the following oxidation step. It was re-dissolved in THF. 
Silver oxide ( $c a .50 \mathrm{mg}$ ) was subsequently added into the solution and the mixture was stirred in air for $12 \mathrm{~h}$. Q1 was purified with flash column chromatography ( $\mathrm{SiO}_{2}$, hexane/dichloromethane 3/1). Q2, Q3 and $\mathbf{Q 4}$ were first purified by flash column chromatography ( $\mathrm{SiO}_{2}$, hexane/ethyl acetate), then further purified by preparation SEC with chloroform as the eluent.

Q1: Dark red solid. (1.03 g, 63\%) ${ }_{1} \mathrm{H}$ NMR (500 MHz, CDCl3, 298K): $\delta=0.70(\mathrm{~m}, 8 \mathrm{H}), 0.78$ (t, $12 \mathrm{H}, J=7.2 \mathrm{~Hz}), 0.94-1.19(\mathrm{~m}, 24 \mathrm{H}), 1.70(\mathrm{~s}, 12 \mathrm{H}), 2.01\left(\mathrm{pd}, 8 \mathrm{H}, J_{1}=13.2 \mathrm{~Hz}, J_{2}=5.0 \mathrm{~Hz}\right), 7.26$ (s, 2H), $7.37(\mathrm{~m}, \mathrm{H}), 7.54$ (s, 2H), 7.77 (d, 2H, $J=7.4 \mathrm{~Hz}), 7.80(\mathrm{~s}, 2 \mathrm{H}) .13 \mathrm{C}$ NMR (125 MHz, $\left.\mathrm{CDCl}_{3}, 298 \mathrm{~K}\right) \delta=154.27,151.83,150.14,142.84,142.26,142.03,140.35,136.36,131.16,127.63$, $126.89,124.41,122.99,119.93,116.84,54.90,40.45,37.05,33.42,31.55,29.77,23.88,22.62$, 13.99. HRMS (+ESI): $\mathrm{C}_{62} \mathrm{H} 78 \mathrm{~N}_{2}[\mathrm{M}+\mathrm{H}]+$ calcd. $m / z=851.6238$, found $m / z=851.6233$.

Q2: Dark-purple powder (0.12 g, 86\%) ${ }_{1} \mathrm{H}$ NMR $\left(500 \mathrm{MHz}, \mathrm{CDCl}_{3}, 298 \mathrm{~K}\right): \delta=0.58-0.73(\mathrm{~m}$, $12 \mathrm{H}), 0.77(\mathrm{t}, 18 \mathrm{H}, J=7.2 \mathrm{~Hz}), 0.97-1.22(\mathrm{~m}, 36 \mathrm{H}), 1.72(\mathrm{~s}, 12 \mathrm{H}), 1.76(\mathrm{~s}, 12 \mathrm{H}), 2.01(\mathrm{~m}, 12 \mathrm{H})$, $7.27(\mathrm{~s}, 2 \mathrm{H}), 7.29(\mathrm{~s}, 2 \mathrm{H}), 7.35(\mathrm{~m}, 6 \mathrm{H}), 7.52(\mathrm{~s}, 2 \mathrm{H}), 7.53(\mathrm{~s}, 2 \mathrm{H}), 7.74(\mathrm{~m}, 2 \mathrm{H}), 7.78(\mathrm{~s}, 2 \mathrm{H}), 7.82$ (s, 2H). ${ }_{13 \mathrm{C} \mathrm{NMR}}\left(125 \mathrm{MHz}, \mathrm{CDCl}_{3}, 298 \mathrm{~K}\right) \delta=154.50,154.18,151.84,151.31,150.17,143.43$, $142.85,142.15,142.09,141.16,140.30,136.70,136.40,135.19,131.32,131.08,127.69,126.91$, $125.00,124.45,122.99,119.95,117.19,116.88,54.89,54.71,40.54,40.44,37.08,33.49,33.42$, $32.19,31.61,31.55,29.82,29.76,26.38,24.01,23.87,23.42,22.67,22.61,13.99$. HRMS (+ESI): $[\mathrm{M}+\mathrm{H}]+$ calcd. $m / z=1368.9781$, found $m / z=1368.9738$.

Q3: Dark-blue powder (20 mg, 72\%) $1 \mathrm{H}$ NMR (500 MHz, CDCl3, 298K): $\delta=0.76(\mathrm{~m}, 40 \mathrm{H}), 0.93-$ $1.18(\mathrm{~m}, 48 \mathrm{H}), 1.57(\mathrm{~s}, 12 \mathrm{H}), 1.70(\mathrm{~s}, 12 \mathrm{H}), 1.74(\mathrm{~s}, 12 \mathrm{H}), 2.01(\mathrm{~m}, 16 \mathrm{H}), 7.27(\mathrm{~s}, 2 \mathrm{H}), 7.29(\mathrm{~s}$, 2H), $7.30(\mathrm{~s}, 2 \mathrm{H}), 7.32-7.39(\mathrm{~m}, 6 \mathrm{H}), 7.53(\mathrm{~s}, 6 \mathrm{H}), 7.75(\mathrm{~d}, 2 \mathrm{H}, J=7.0 \mathrm{~Hz}), 7.78(\mathrm{~s}, 2 \mathrm{H}), 7.82(\mathrm{~s}$, 4H). ${ }_{13} \mathrm{C} \mathrm{NMR}\left(125 \mathrm{MHz}, \mathrm{CDCl}_{3}, 298 \mathrm{~K}\right) \delta=154.53,154.43,154.16,153.30,151.84,151.34$ $150.17,144.40,143.46,142.84,142.44,142.17,142.07,141.39,141.29,141.12,140.30,136.75$, 
$136.41,131.35,131.25,131.07,127.68,126.93,124.51,124.44,123.00,119.96,117.23,116.89$,

$54.89,54.71,40.55,40.45,37.13,33.50,33.42,31.62,31.55,29.72,24.02,23.87,22.68,22.62$,

14.01. HRMS(+ESI): $\mathrm{C}_{136} \mathrm{H}_{166} \mathrm{~N}_{6}[\mathrm{M}+2 \mathrm{H}]_{2+}$ calcd. $m / z=943.6660$, found $m / z=943.6690$.

Q4: Dark-blue powder (27 mg, 73\%). $1 \mathrm{H} \mathrm{NMR} \mathrm{(500} \mathrm{MHz,} \mathrm{CDCl3,} \mathrm{298K):} \delta 7.83$ (m, 8H), 7.78 $(\mathrm{d}, J=5.7 \mathrm{~Hz}, 2 \mathrm{H}), 7.56(\mathrm{~m}, 8 \mathrm{H}), 7.36(\mathrm{~m}, 6 \mathrm{H}), 7.33-7.21(\mathrm{~m}, 8 \mathrm{H}), 2.05(\mathrm{~m}, 20 \mathrm{H}), 1.75(\mathrm{~m}, 48 \mathrm{H})$, 1.22-1.00 (m, 60H), $0.79(\mathrm{~m}, 50 \mathrm{H}) .{ }_{13} \mathrm{C}$ NMR (126 MHz, d8-THF, 298K) $\delta=155.07,155.04$, $155.03,154.89,152.21,151.46,151.43,150.36,144.76,144.71,144.30,142.66,142.60,142.55$ $142.54,142.45,142.16,142.08,141.56,141.47,137.40,137.37,137.09,131.94,131.82,131.44$ $131.08,128.95,128.13,127.92,127.52,125.34,123.46,120.70,118.21,117.63,55.44,55.18$, $41.08,37.59,33.39,32.38,32.32,30.63,30.53,23.39,23.31,14.18$. HRMS (+MALDI-TOF): $\mathrm{C}_{173} \mathrm{H}_{210 \mathrm{~N}}[\mathrm{M}+\mathrm{H}]+$ calcd. $m / z=2401.6785$, found $\mathrm{m} / \mathrm{z}=2401.4970$.
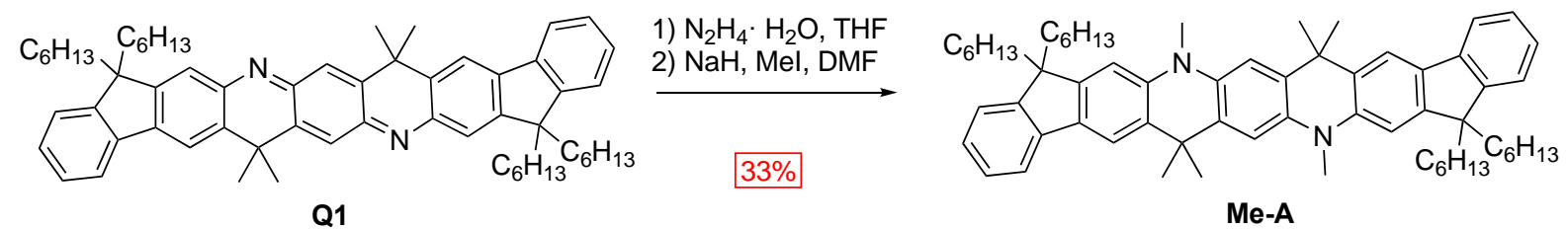

Scheme S9. Synthesis of Me-A from Q1

Me-A: Q1 (0.45 g, $0.53 \mathrm{mmol})$ was dissolved in degassed THF under $\mathrm{N}_{2}$ protection. $\mathrm{N}_{2} \mathrm{H}_{4} \cdot \mathrm{H}_{2} \mathrm{O}$ $(0.26 \mathrm{~mL}, 5.3 \mathrm{mmol})$ was added slowly and the mixture was stirred at $40{ }^{\circ} \mathrm{C}$ for $12 \mathrm{~h}$. The solution turned from red to light yellow. It was then cooled to room temperature and the solvent and excess hydrazine was removed under reduced pressure. After dried under vacuum, the crude product was charged into the next step without further purification. It was redissolved in $10 \mathrm{~mL}$ anhydrous DMF and sodium hydride ( $88 \mathrm{mg}, 60 \%, 2.2 \mathrm{mmol}$ ) was added. The mixture was heat to $60{ }^{\circ} \mathrm{C}$ and stirred for $1 \mathrm{~h}$. After cooling to $35^{\circ} \mathrm{C}$, methyl iodide $(0.23 \mathrm{~mL}, 4.1 \mathrm{mmol})$ was added slowly into the mixture. The reaction was stirred for $12 \mathrm{~h}$ before being quenched by water. The mixture was 
then extracted by dichloromethane for 3 times. The combined organic layer was washed by water. After dried against magnesium sulfonate, the crude product was purified using flash column $\left(\mathrm{SiO}_{2}\right.$, hexane/ethyl acetate 9:1). The pure product was isolated as a white powder with purple fluorescence (0.13 g, 33\%). $1 \mathrm{H}$ NMR $\left(500 \mathrm{MHz}, \mathrm{CDCl}_{3}\right) \delta 7.70(\mathrm{~s}, 2 \mathrm{H}), 7.64(\mathrm{~d}, J=7.5 \mathrm{~Hz}, 4 \mathrm{H})$, 7.29 (m, 4H), 7.18 (t, J= $7.3 \mathrm{~Hz}, 2 \mathrm{H}), 7.09$ (s, 2H), 6.83 (s, 2H), 3.57 (s, 6H), 1.93 (m, 8H), 1.65 (s, 12H), $1.17-0.94(\mathrm{~m}, 24 \mathrm{H}), 0.75(\mathrm{t}, J=7.1 \mathrm{~Hz}, 12 \mathrm{H}), 0.69-0.52(\mathrm{~m}, 8 \mathrm{H}) .13 \mathrm{C} \mathrm{NMR}(125 \mathrm{MHz}$ $\left.\mathrm{CDCl}_{3}\right) \delta=150.35,149.59,142.96,142.09,136.95,133.43,131.60,131.25,126.67,125.33$, $122.75,118.43,114.90,108.18,106.43,54.99,40.82,37.10,34.11,31.66,29.96,27.61,23.87$, 22.78, 14.16. $\mathrm{HRMS}(+\mathrm{ESI}): \mathrm{C}_{64} \mathrm{H}_{84} \mathrm{~N}_{2}[\mathrm{M} \cdot]+$ calcd. $m / z=880.6629$, found $m / z=880.6610$.

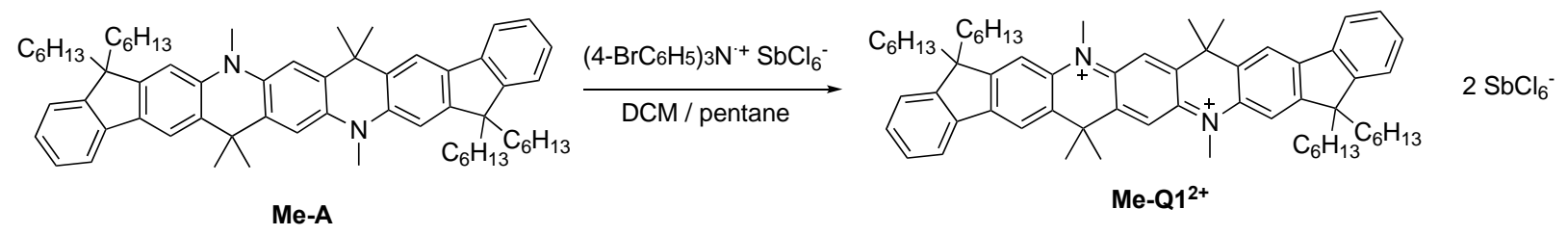

Scheme S10. Synthesis of Me-Q12+·2SbCl6-from Me-A.

Me-Q12+2SbCl6-: Me-A (10.7 mg, $0.01 \mathrm{mmol})$ was dissolved in anhydrous DCM (3 mL). Tris(4bromophenyl)ammoniumyl hexachloroantimonate ("magic blue", $18.4 \mathrm{mg}, 0.022 \mathrm{mmol}$ ) was added into the solution and the mixture was stirred in a nitrogen-filled glovebox for $10 \mathrm{~min}$, turning the color from color-less into deep-blue. The solution was filtered through a syringe filter (PTFE, $0.45 \mu \mathrm{m}$ ) into a clean $20 \mathrm{~mL}$ vial and pentane was then layered above. The single crystal was obtained after diffusion for 2 days and collected by filtration.

\section{COSY NMR Spectroscopy of NLQ in Solution}

${ }_{1} \mathrm{H}-1 \mathrm{H}$ COSY NMR spectrum of $\mathbf{N L Q}$ was conducted in $\mathrm{CD}_{2} \mathrm{Cl}_{2}$ at room temperature. The presence of dinstinct tran- and cis-configurational isomers was observed. The peaks were assigned to each isomer based on the $1 \mathrm{H}-1 \mathrm{H}$ coupling. The molar percentage of $E$ and $Z$ isomers were set to be $x$ and 
$y$, respectively. Based on the NMR integral of each peak, $x$ and $y$ were determined to be $58 \%$ and $42 \%$.

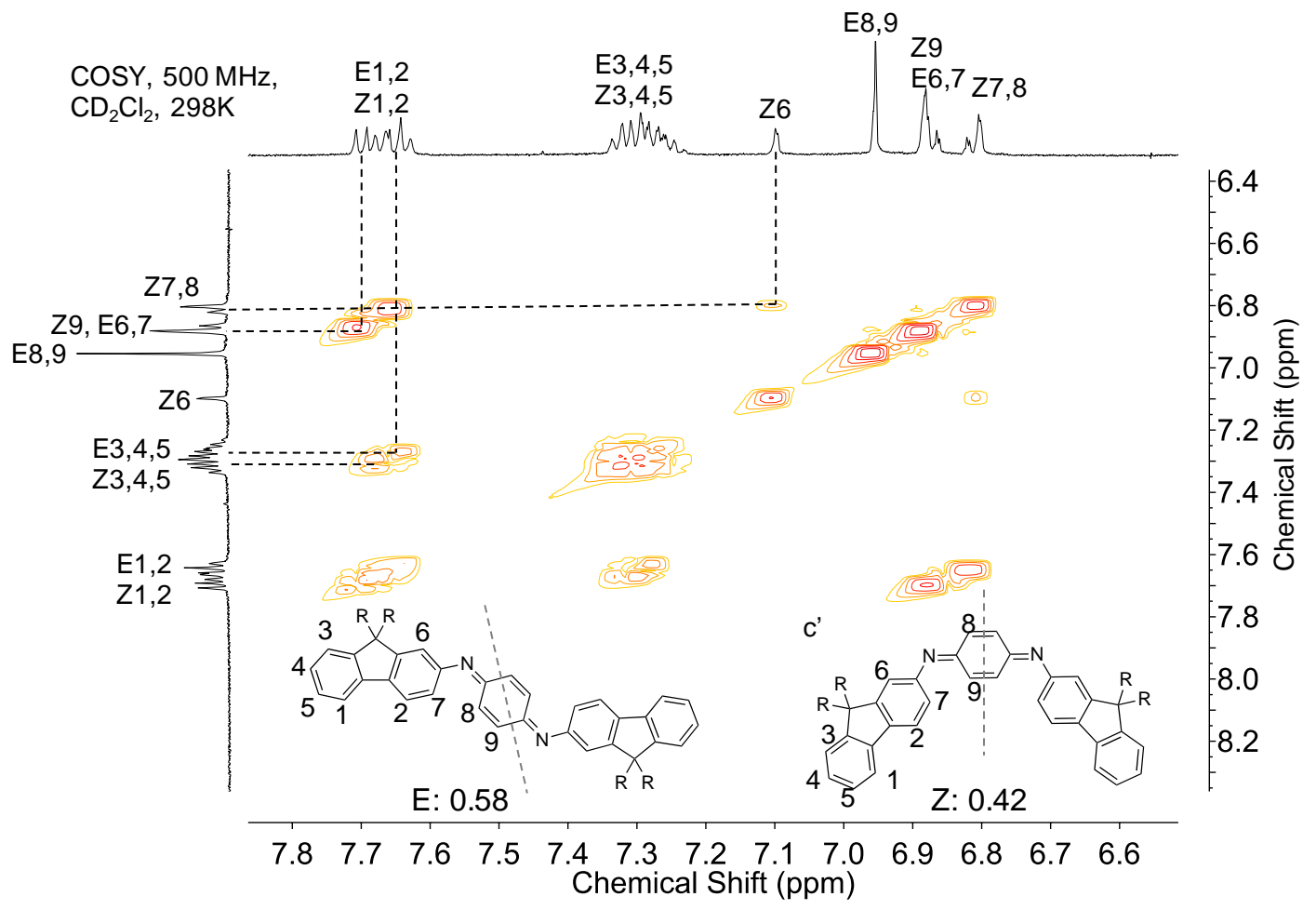

\begin{tabular}{cc} 
Peak & Integral (theoretical proton numbers) \\
$\mathrm{E} 1,2+\mathrm{Z} 1,2$ & $2(2 x+2 y)$ \\
$\mathrm{E} 3,4,5+\mathrm{Z} 3,4,5$ & $3.2(3 x+3 y)$ \\
$\mathrm{Z} 6$ & $0.42(y)$ \\
$\mathrm{E} 8,9$ & $1.13(2 x)$ \\
$\mathrm{E} 6,7+\mathrm{Z} 9$ & $1.60(2 x+y)$ \\
$\mathrm{Z} 7,8$ & $0.87(2 y)$ \\
\hline
\end{tabular}

Figure S1. COSY spectrum of NLQ in $\mathrm{CD}_{2} \mathrm{Cl}_{2}$ at room temperature and the peak assignments.

The integral of peak $(\mathrm{E} 1,2+\mathrm{Z1} 1,2)$ was set as 2 protons. The molar percentage of $E$ and $Z$ isomers were set to be $x$ and $y$. 


\section{Determination of Energy Levels of Frontier Molecular Orbitals by Electrochemical Analysis and Density Functional Theory Calculation}

The redox properties of oligomers Q1 Q4 and the corresponding energy levels of frontier orbitals were determined by cyclic voltammetry (CV) and differential pulse voltammetry (DPV) carried out at room temperature under argon protection with a $\mathrm{CHI} 720$ voltammetric analyzer. $n$-Bu4 $\mathrm{PF}_{6}$ in dichloromethane $(0.1 \mathrm{~mol} / \mathrm{L})$ was used. A conventional three-electrode configuration was used, including a $3 \mathrm{~mm}$ glassy carbon working electrode, a platinum wire auxiliary electrode, and an $\mathrm{Ag}$ wire pseudoreference electrode with electrolyte solution $\left(\mathrm{AgNO}_{3} 10 \mathrm{mmol} / \mathrm{L}\right.$ in dichloromethane $)$ freshly prepared in a $\mathrm{N}_{2}$ glovebox right before the measurement. Scan rate was $0.1 \mathrm{~V} / \mathrm{s}$ for $\mathrm{CV}$ test and $0.04 \mathrm{~V} / \mathrm{s}$ for DPV test. Ferrocene was used as an exterior reference for determination of potentials and energy levels.

Density functional theory (DFT) calculations of the atomic structures of Q1 Q4 were optimized at B3LYP/6-311G(d,p) level of theory. Molecular orbital shapes and energies were calculated based on single crystal structure of $\mathbf{Q 1}$ or the optimized geometries $(\mathbf{Q 2} \mathbf{Q 4})$. The alkyl chains are replaced with methyl groups for computational simplicity. Orbital pictures were generated with Gaussview 5.08. 

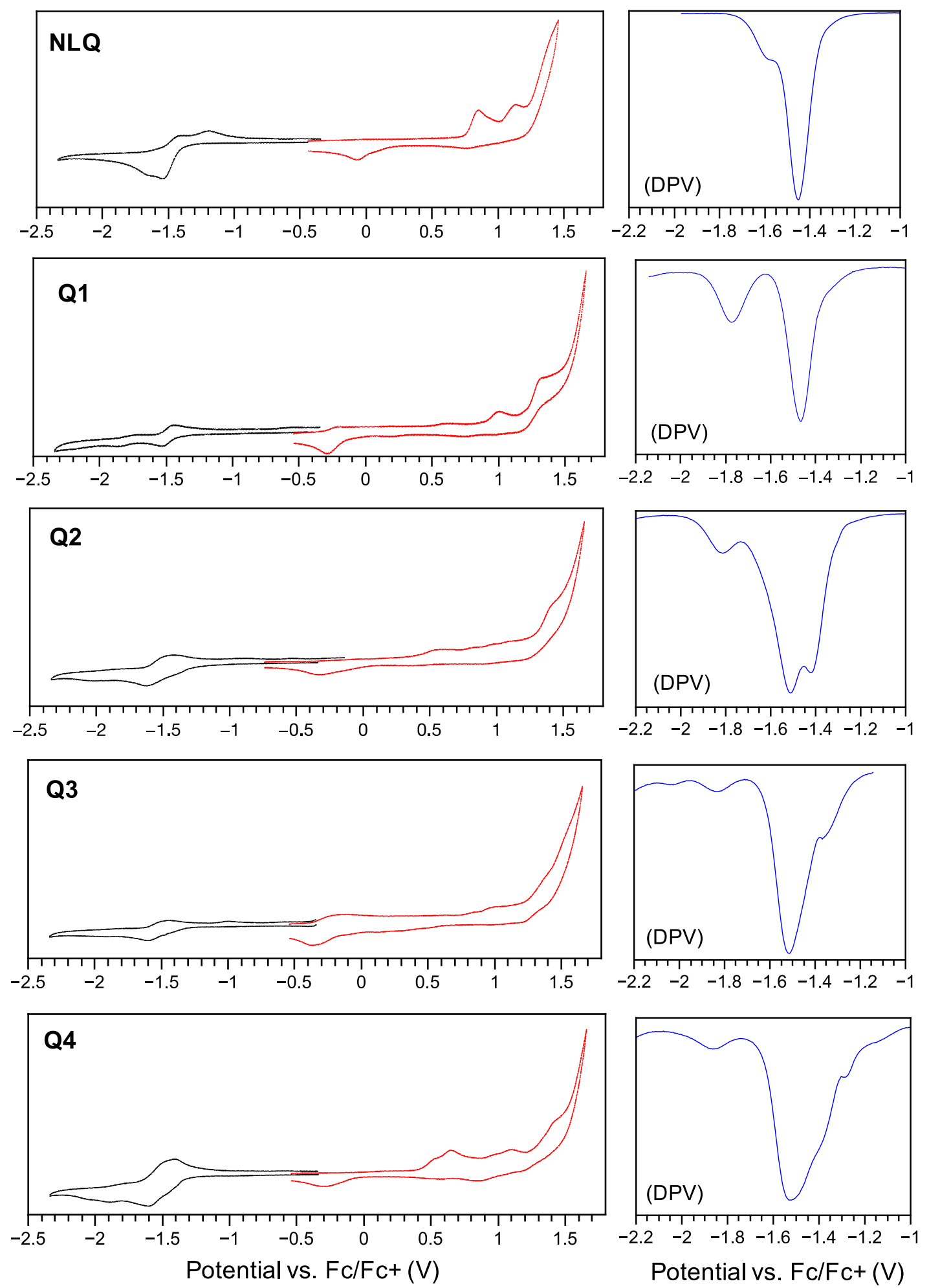

Figure S2. CV and DPV traces of Q1 Q4 and NLQ. 
All of these ladder oligomers demonstrated quasi-reversible multi-electron reduction processes, while that of the control compound NLQ was irreversible. This observation indicated that the ladder constitution better stabilized the anionic species upon reduction.

Table S2. Measured and Calculated HOMO and LUMO Levels of Q1 Q4

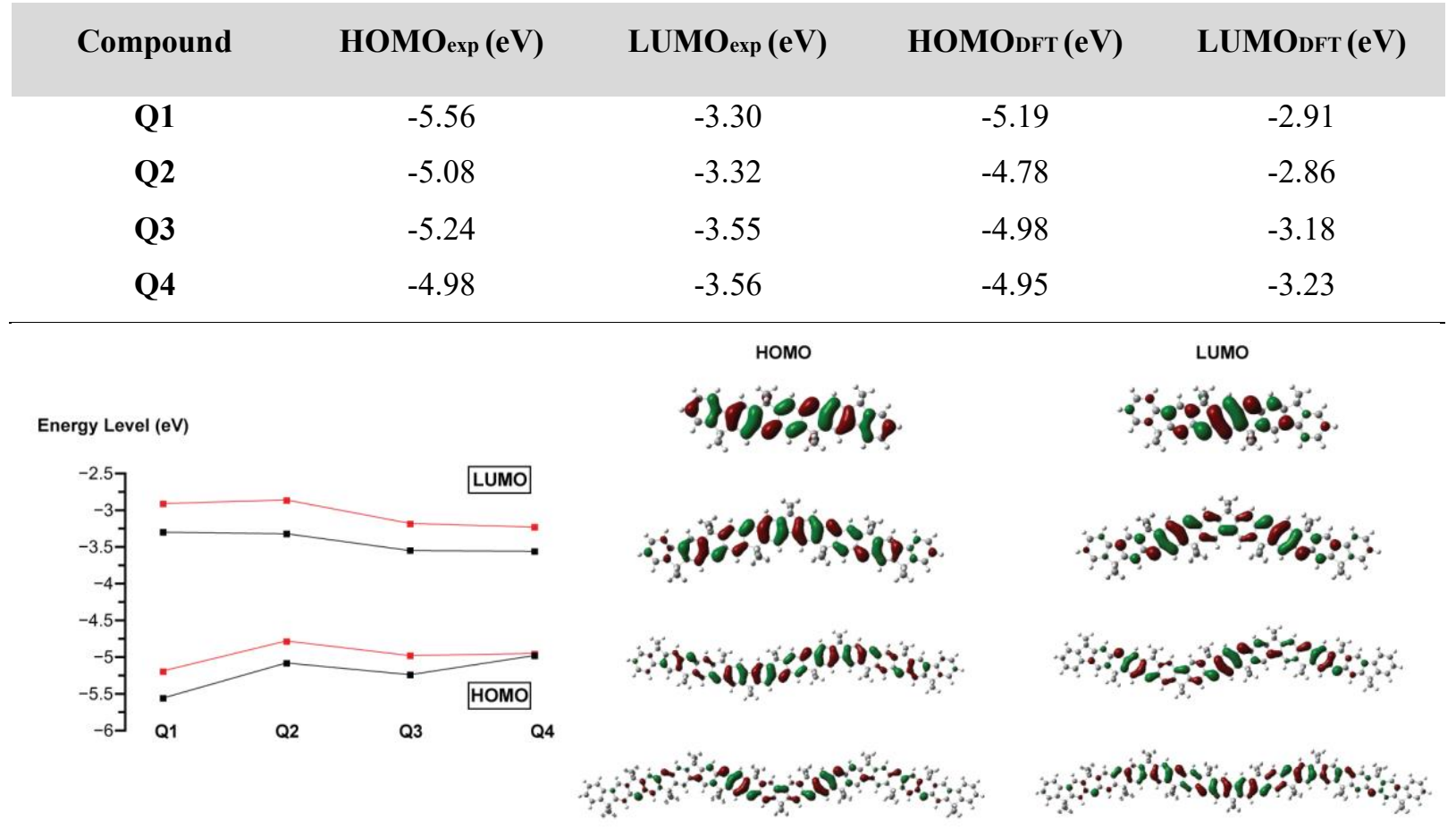

Figure S3. (left) Energy levels of frontier orbitals of Qn $(n=1 \sim 4)$ measured by CV (black) and calculated using DFT (red); (right) Diagrams of HOMOs and LUMOs of $\mathbf{Q n}(\mathrm{n}=1 \sim 4)$ generated by DFT calculation.

\section{UV-vis-NIR Absorption Spectra Before and After Protonation}

The well-defined structure of the ladder-type backbone of Q1 Q4 was further confirmed by the ladder-type backbone rigidity was expected to impact the optical properties of Q1 Q4 significantly. All the absorption spectra of Q1 Q4 demonstrated distinctive vibrational progressions owing to their conformational rigidity. In a sharp contrast, the control compound NLQ, with a flexible 
backbone, exhibited a broad absorption peak at around $500 \mathrm{~nm}$, similar to that of poly(pernigraniline base), indicative of its flexible conformation and isomerizing configuration. The maximum absorption wavelengths of Q1 to Q4 red-shifted from $553 \mathrm{~nm}$ to $680 \mathrm{~nm}$ with the increase of the oligomer sizes due to the increased extension of $\pi$-conjugation and the consequent decreased energy bandgaps. A linear relationship with $\mathrm{R}_{2}=0.99$ was found when plotting the optical energy bandgap (Eg, optical) versus the reciprocal of repeating unit numbers $(\mathrm{n}-1)$, similar to other reported rigid polycyclic aromatic systems.

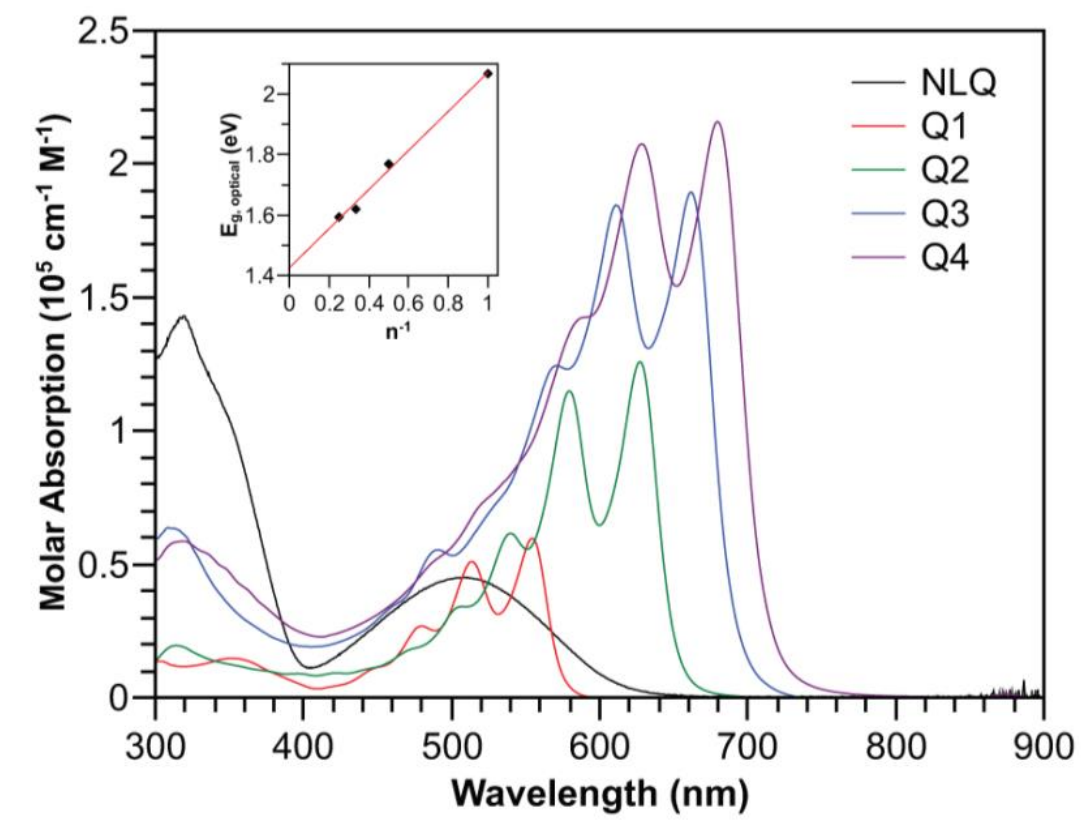

Figure S4. UV-vis absorption spectra of NLQ and $\mathbf{Q n}(\mathrm{n}=1 \sim 4)$ in toluene $(10-5 \mathrm{M})$ at room temperature. Inset: the plot of optical band gap versus $\mathrm{n}-1(\mathrm{n}=1 \sim 4)$.

To record the optical spectra of NLQ after protonation, NLQ was dissolved in THF and diluted into a solution of $1.1 \times 10-5 \mathrm{~mol} / \mathrm{L}$. UV-vis absorption of NLQ solution $(2.5 \mathrm{~mL})$ was measured in a 1-cm quartz cuvette. Methanesulfonic acid (MSA) was dissolved in THF $(1.54 \mathrm{~mol} / \mathrm{L})$ and added into the cuvette in portions of $2 \mu \mathrm{L}, 4 \mu \mathrm{L}, 6 \mu \mathrm{L}, 10 \mu \mathrm{L}, 20 \mu \mathrm{L}, 30 \mu \mathrm{L}, 50 \mu \mathrm{L}, 70 \mu \mathrm{L}, 110 \mu \mathrm{L}, 150$ $\mu \mathrm{L}$, and $250 \mu \mathrm{L}$. For high acid concentrations, NLQ was dissolved in nitrogen-bubbled solutions 
of MSA in THF $(0.15,1.54,3.08,7.7$, and $15.4 \mathrm{~mol} / \mathrm{L})$ and measured.

The protonation experiment was performed in the similar set-up for Q1. During the first protonation stage, A solution of MSA in THF (0.5 mol/L) was added into Q1 solution (2.5 mL, 2.2 $\times 10-5 \mathrm{~mol} / \mathrm{L})(1.5 \mu \mathrm{L}, 2.5 \mu \mathrm{L}, 3.5 \mu \mathrm{L}, 4.5 \mu \mathrm{L}, 5.5 \mu \mathrm{L}, 6.5 \mu \mathrm{L}, 8.5 \mu \mathrm{L}, 25 \mu \mathrm{L}$, and $45 \mu \mathrm{L})$. During the second protonation stage, Q1 was dissolved in a mixed solvent of MSA in THF (1.5 mol/L), affording a fully protonated Q1 solution $(2.2 \times 10-5 \mathrm{~mol} / \mathrm{L})$. This solution was added into the neutral Q1 solution $(1 \mathrm{~mL})$ of the same concentration in steps of $10 \mu \mathrm{L}, 30 \mu \mathrm{L}, 90 \mu \mathrm{L}, 150 \mu \mathrm{L}$, $210 \mu \mathrm{L}, 290 \mu \mathrm{L}, 350 \mu \mathrm{L}, 450 \mu \mathrm{L}, 650 \mu \mathrm{L}$, and $910 \mu \mathrm{L}$.
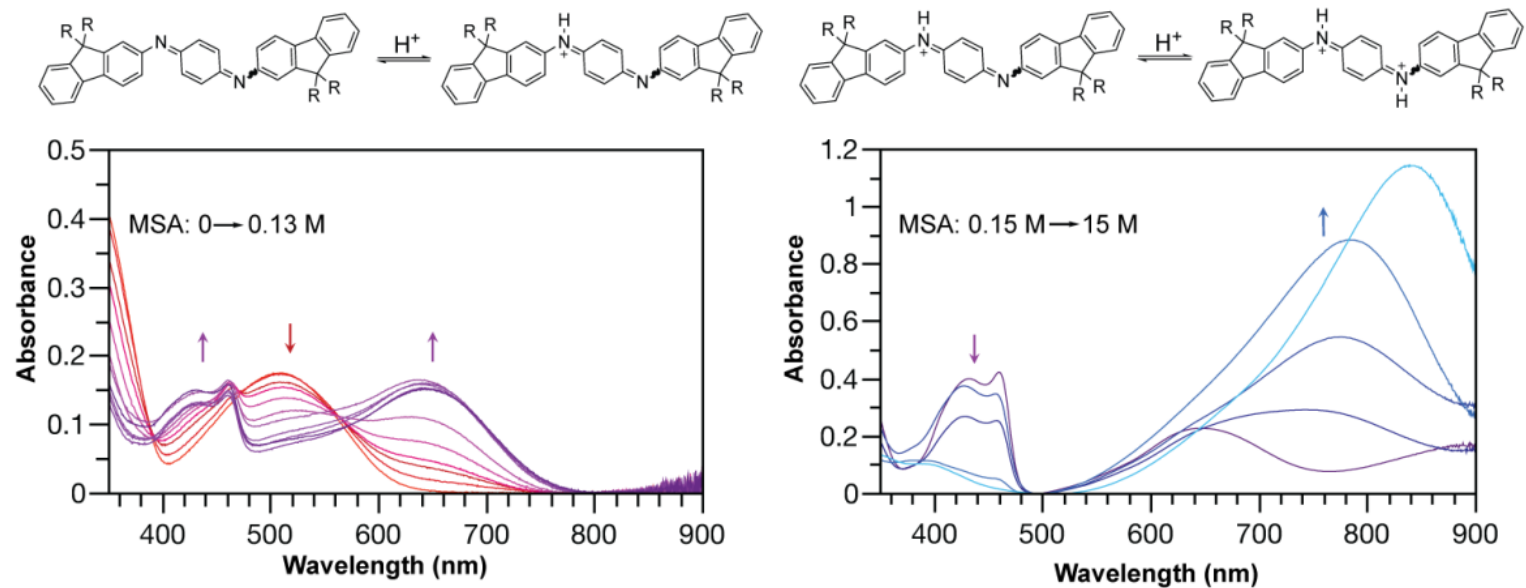

Figure S5. Structural formulas and UV-vis absorption spectra corresponding to the protonation stages of NLQ in THF solution (10-5 mol/L) with increasing amount of MSA.

p-Toluenesulfonic acid (PTSA) was used for solid-state acid-doping. The acid was prepared into a THF solution. Qn was dissolved in the PTSA solutions of varies volumes as shown in Table $\mathrm{S} 3$. The resulting mixtures were drop-cast on a glass slide and dried in a desiccator for over $12 \mathrm{~h}$ under reduced pressure before measured using UV-vis-NIR spectroscopy. 
Table S3 Preparation of Protonated Qn in the Solid State

\begin{tabular}{ccccc} 
Compound & $\begin{array}{c}\text { Mole (10-5 } \\
\text { mmol) }\end{array}$ & $\begin{array}{c}\text { Volume of PTSA } \\
\text { Solution }(\mathbf{m L})\end{array}$ & $\begin{array}{c}\text { Concentration of } \\
\text { PTSA Solution } \\
(\mathbf{m o l} / \mathbf{L})\end{array}$ & $\begin{array}{c}\text { Molar Ratio } \\
\text { (Qn : PTSA) }\end{array}$ \\
\hline \multirow{2}{*}{ Q1 } & 2.7 & 0.30 & 0.010 & $1: 1$ \\
& 23.3 & 0.10 & 0.480 & $1: 2$ \\
\hline \multirow{2}{*}{$\mathbf{Q} 2$} & 24.0 & 0.25 & 0.480 & $1: 5$ \\
& 24.1 & 1.00 & 0.480 & $1: 20$ \\
\hline Q3 & 1 & 0.10 & 0.040 & $1: 4$ \\
\hline Q4 & 1 & 0.25 & 0.040 & $1: 10$ \\
\hline
\end{tabular}
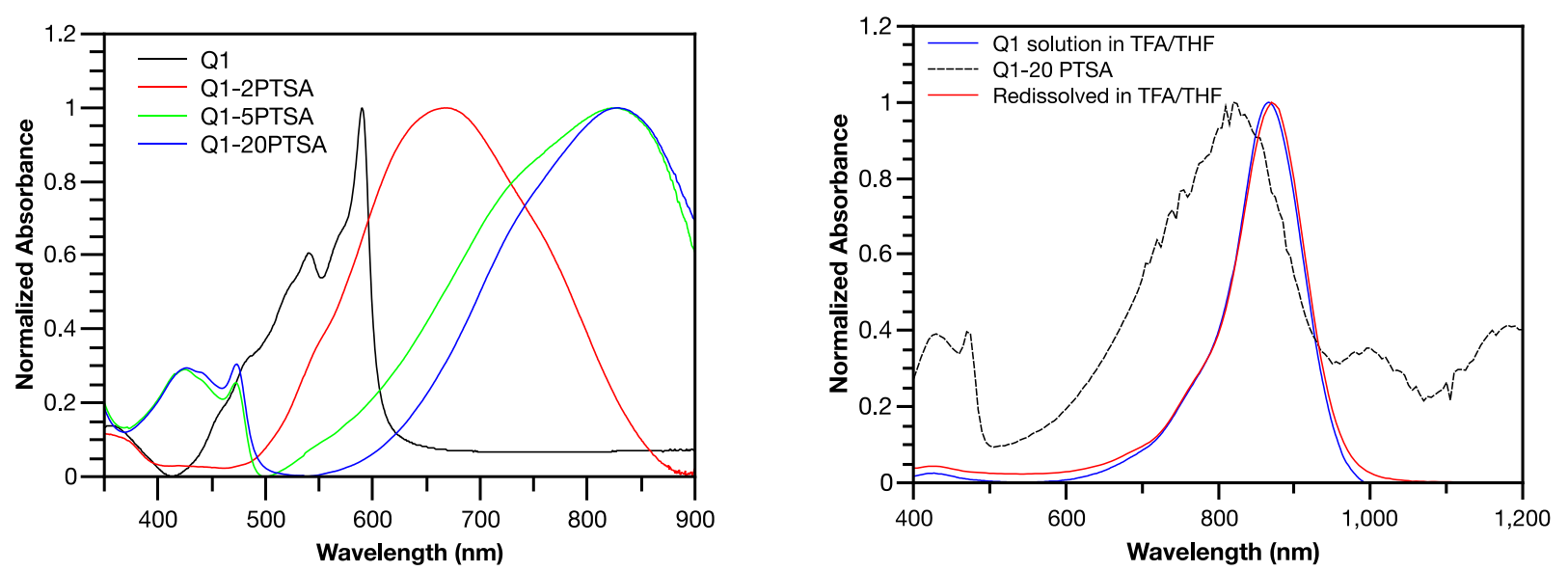

Figure S6. (left) Solid state UV-vis absorption spectra of Q1 with varied equivalences of PTSA in thin films; (right) Normalized UV-vis-NIR absorption spectra of Q1 solution (10-5 mol/L) in THF with $0.44 \mathrm{~mol} / \mathrm{L}$ TFA (red), Q1 with 20 equivalences of PTSA thin film (black), and the solution prepared by re-dissolving the film in THF with $0.44 \mathrm{~mol} / \mathrm{L}$ TFA (blue). 

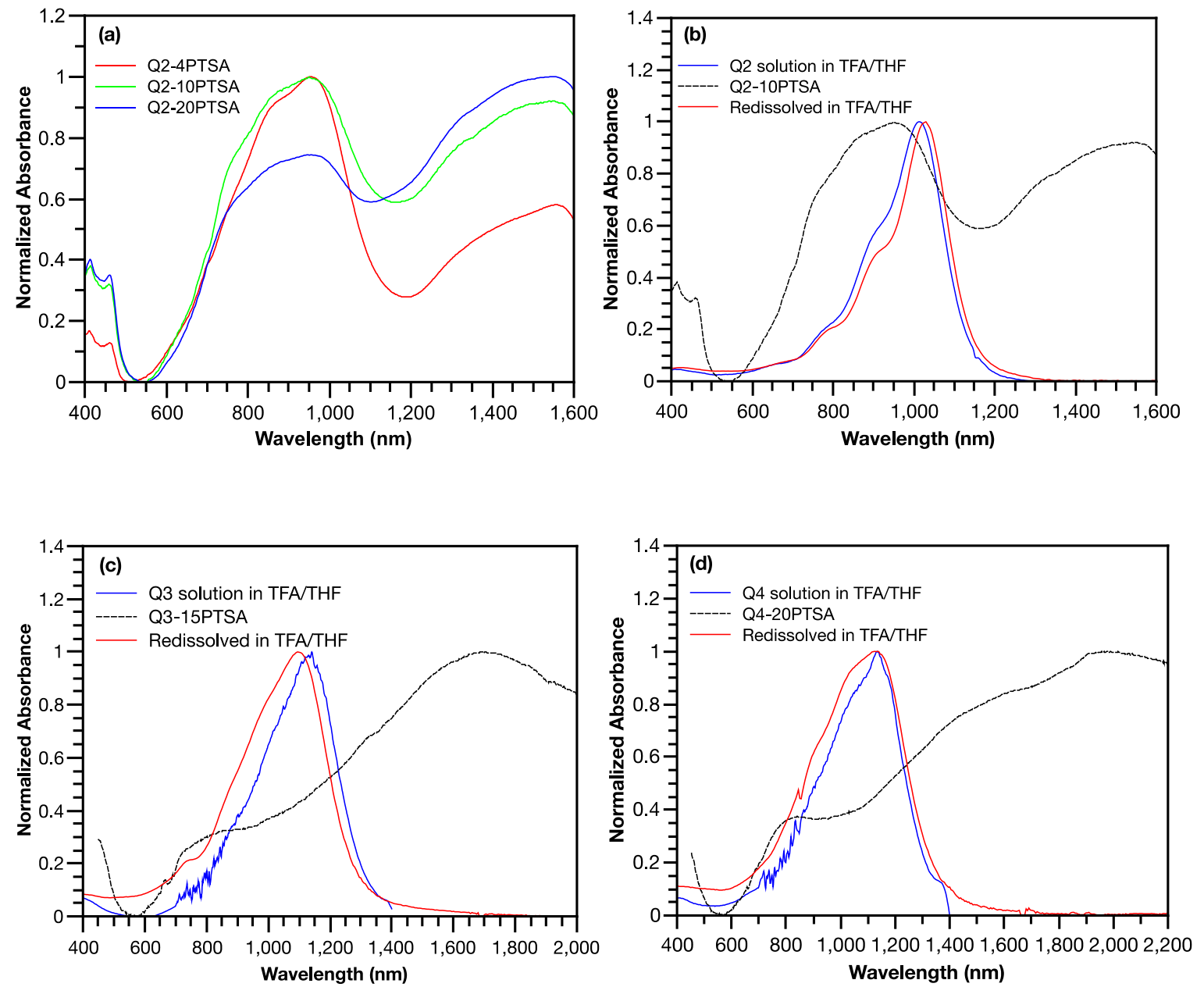

Figure S7. UV-vis-NIR absorption spectra of (a) Q2 with varies equivalences of PTSA in thin films and (b)(c)(d) Qn solution (10-5 mol/L) in THF with $0.44 \mathrm{~mol} / \mathrm{L}$ TFA (blue), Qn with $5 \mathrm{n}$ equivalences of PTSA thin film (black), and the solution prepared by dissolving the film in THF with $0.44 \mathrm{~mol} / \mathrm{L}$ TFA (red). $(\mathrm{n}=2,3,4)$ 


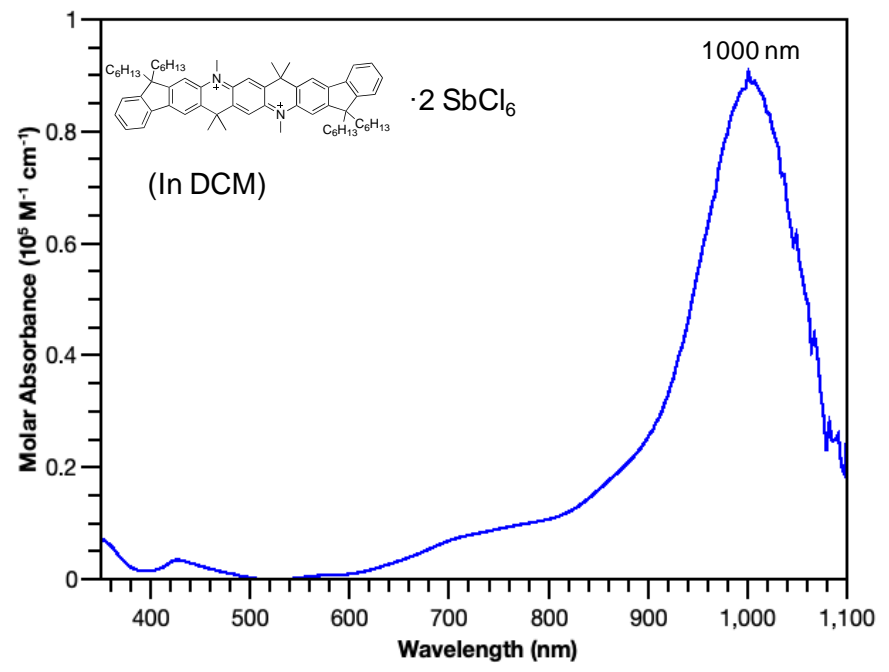

Figure S8. UV-vis-NIR absorption spectrum of Me-Q12+·2 SbCl6- in dichloromethane.

\section{Degradation Study}

The degradation study of Qn and NLQ was performed with UV-vis absorption measurement. Solutions $(c a .1 \times 10-5 \mathrm{~mol} / \mathrm{L})$ were prepared by dissolving Qn and NLQ in a solution of MSA in THF $(0.8 \mathrm{~mol} / \mathrm{L})$. UV-vis absorption was measured every $5 \mathrm{~min}$ at room temperature at ambient condition for 70 min. NLQ showed continuously decreased absorption signal intensity while all Qn showed stable absorption peaks without significant changes.

The structural integrity of $\mathbf{Q 1}$ after protonation was further confirmed by NMR spectroscopy. $1 \mathrm{H}$ NMR spectrum of $\mathbf{Q} 1$ in TFA/CDCl3 $(0.44 \mathrm{~mol} / \mathrm{L})$ remained identical after $24 \mathrm{~h}$. The basic form of Q1 was subsequently regenerated by adding $\mathrm{KOH}$ into the solution, showing a $1 \mathrm{H}$ NMR spectrum identical to that of $\mathbf{Q 1}$ before the acid/base treatment. These results confirmed that protonated Q1 did not undergo significant decomposition or redox transformation in ambient conditions in the time scale of hours. 

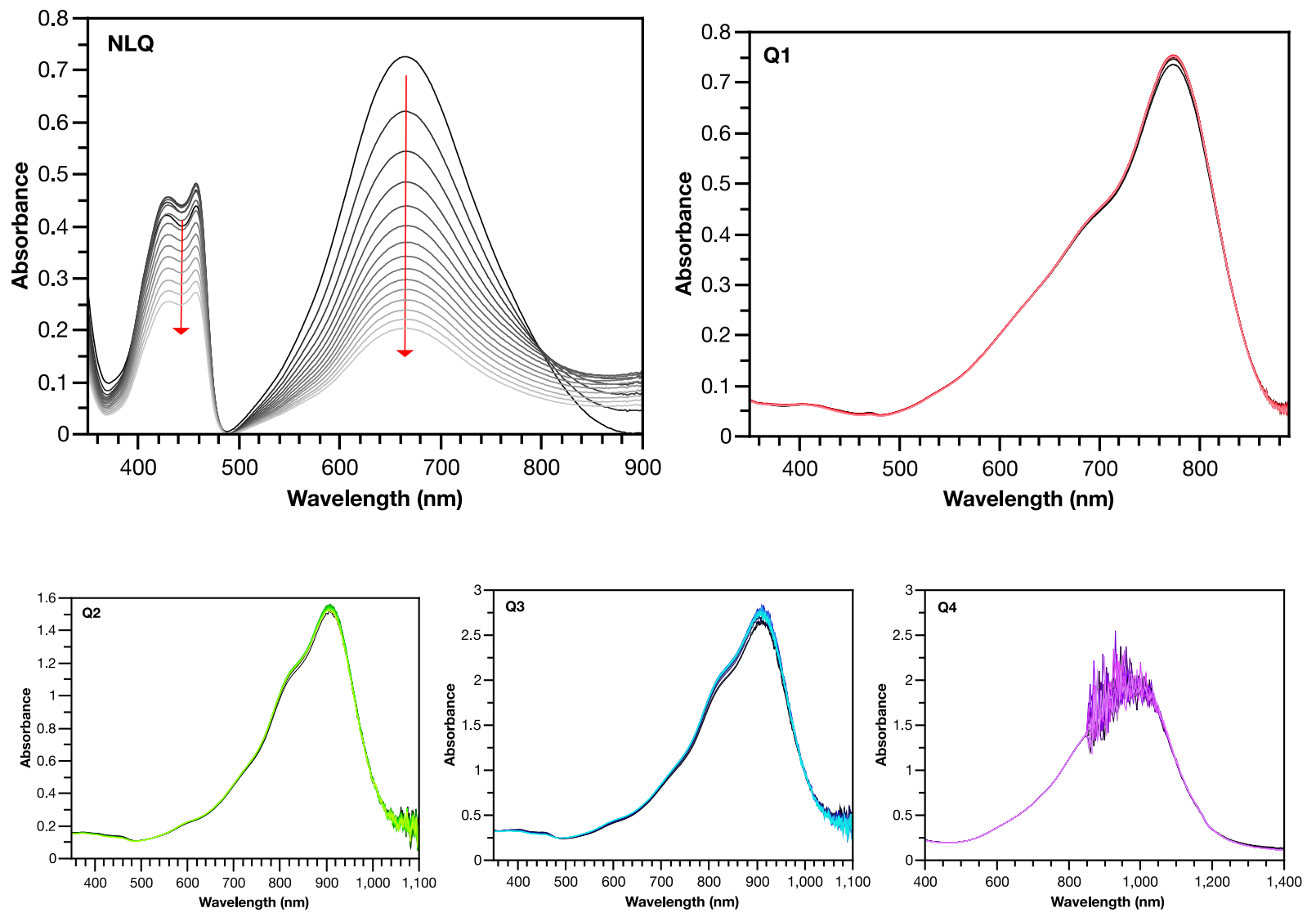

Figure S9. Time-dependent UV-vis spectra of protonated NLQ and Qn in MSA/THF (0 70 min). 


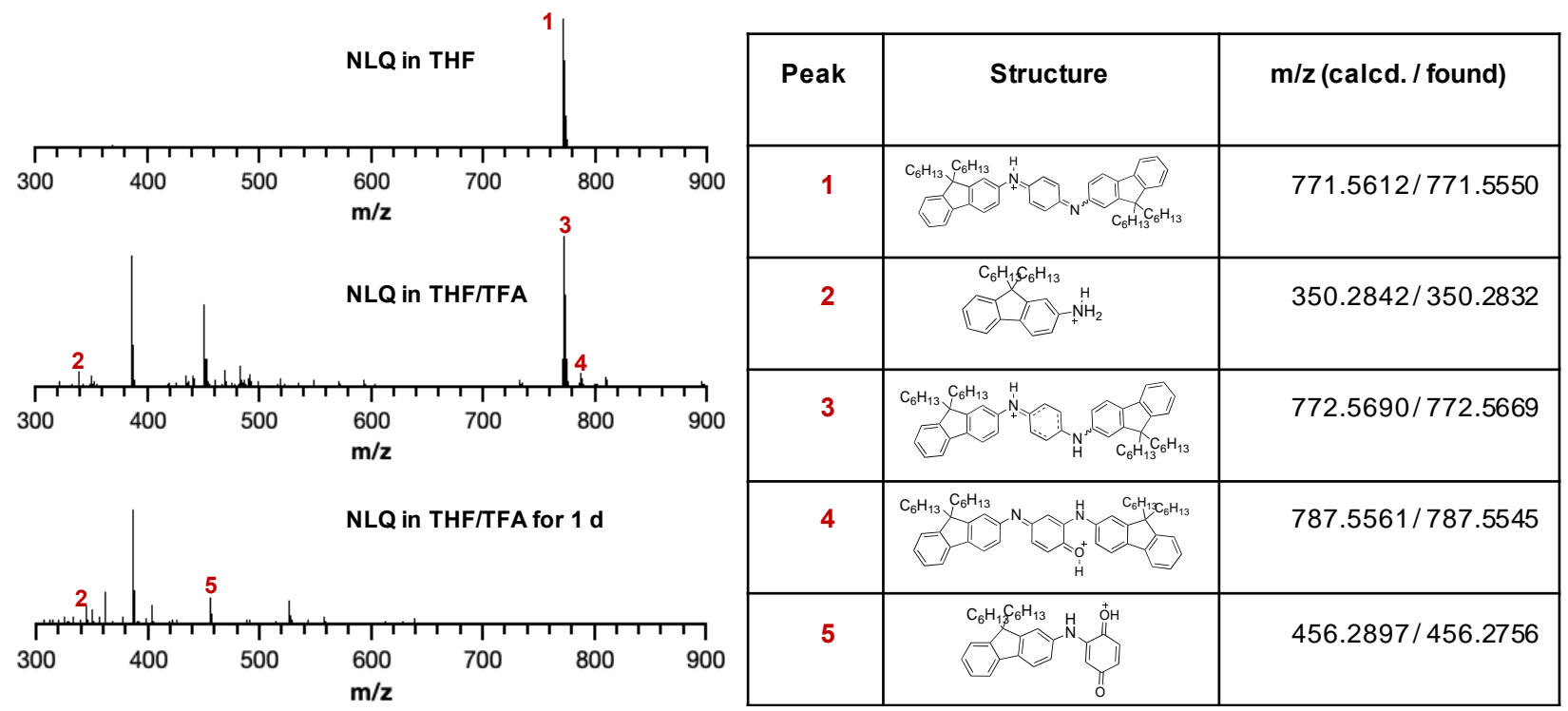

Figure S10. High-resolution mass spectra of NLQ in THF with TFA (v/v = 10/1) and peak assignments. The reduced product (Peak 1), the hydrolyzed amino product (Peak 2), and the rearranged product (Peak 4 and 5) was observed. After $1 \mathrm{~d}$, only low molecular weight species were present.

a)

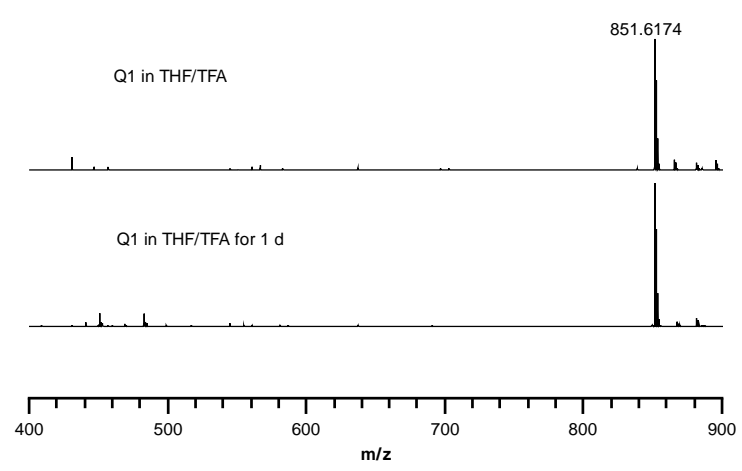

b)

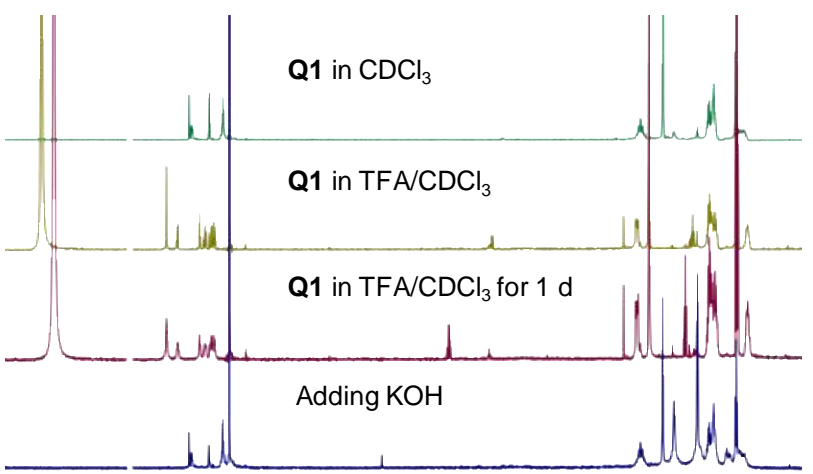

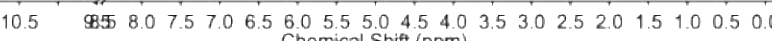

Figure S11. a) High-resolution mass spectra of Q1 in THF with TFA (v/v = 10/1). b) ${ }_{1} \mathrm{H}$ NMR (500 $\mathrm{MHz})$ spectra of $\mathbf{Q 1}$ in $\mathrm{CDCl}_{3}$, adding TFA (v/v = 1/1), after $1 \mathrm{~d}$, and after adding $\mathrm{KOH}$.

\section{Diradical Character Calculation}

H2-Q12+ and H2-NLQ2+ was optimized at the DFT UB3LYP/6-311+G(d,p) level of theory with S25 
broken-symmetry. Q1 and Me-Q12+ was calculated based on the single crystal structure without optimization. The occupancy number of highest occupied natural orbital (HONO) $(\sigma \mathrm{HONO})$ and lowest unoccupied natural orbital (LUNO) ( $\sigma$ LUNO), and corresponding diradical character $y$ were calculated for Q1, $\mathbf{H}_{2}-\mathbf{Q 1 2 +}, \mathbf{H}_{2}-\mathrm{NLQ}_{2+}$ and $\mathbf{M e - Q 1 2 +}$ at $\mathrm{UHF} / 6-31+\mathrm{G}(\mathrm{d}, \mathrm{p})$ level of theory.1 Pernigraniline salt dimer (PS-dimer) was also calculated at the same level of theory.

Table S4. Calculated diradical character of Q1, H2-Q12+, $\mathbf{H}_{2}-\mathrm{NLQ}_{2+}, \mathrm{Me}-\mathrm{Q1} \mathbf{2}_{2}$ and PS-dimer

\begin{tabular}{cccc} 
Compound & $\sigma_{\text {HONO }}$ & $\sigma_{\text {LUNO }}$ & $y$ \\
\hline $\mathbf{Q 1}$ & 1.3759 & 0.6241 & 0.34 \\
H2-Q12+ $_{2+}$ & 1.1830 & 0.8169 & 0.65 \\
H2-NLQ2+ $_{2+}$ & 1.1554 & 0.8446 & 0.70 \\
Me-Q12+ & 1.2618 & 0.7382 & 0.51 \\
PS-dimer & 1.3195 & 0.6805 & 0.42 \\
\hline
\end{tabular}

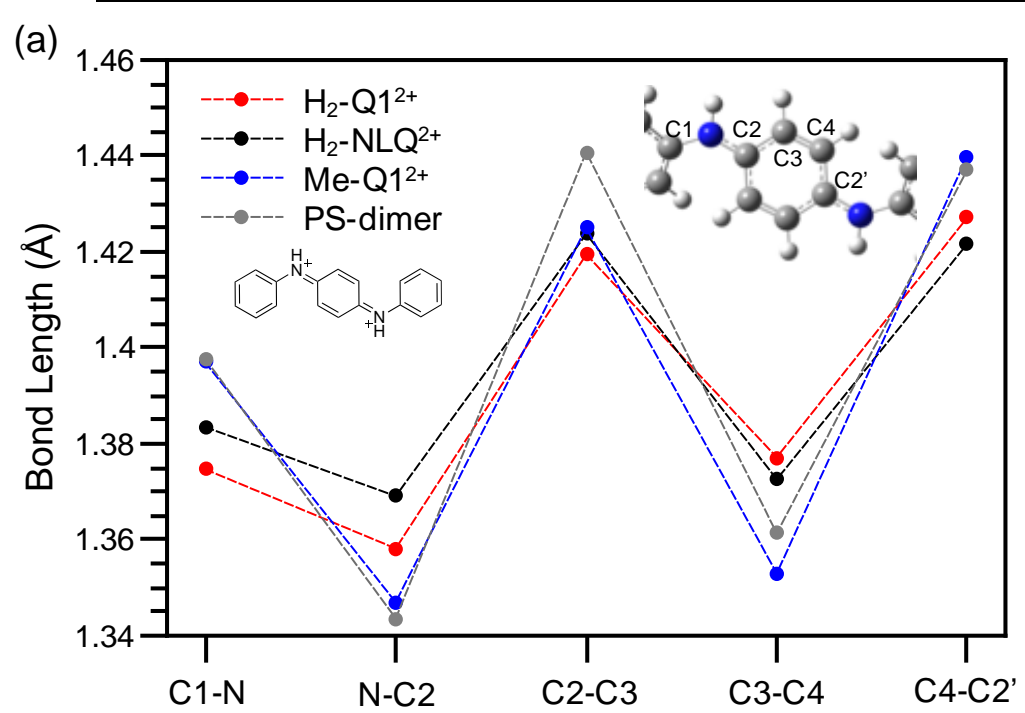

(b) $\mathrm{H}_{2}-\mathrm{Q} 1^{2+}$
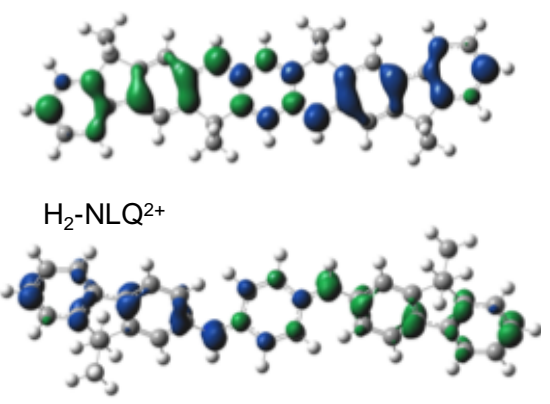

$\mathrm{Me}-\mathrm{Q} 1^{2+}$

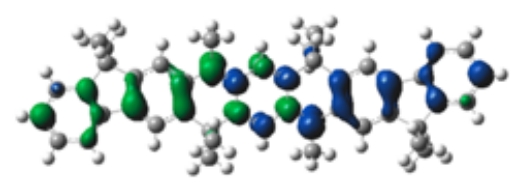

Figure S12. (a) Bond length alternation of $\mathrm{H}_{2}-\mathbf{Q} 1_{2+}, \mathrm{H}_{2}-\mathrm{NLQ}_{2+}, \mathrm{Me}-\mathrm{Q} 12+$ and PS-dimer; (b) spin density distribution of $\mathbf{H}_{2}-\mathbf{Q 1}_{2+}, \mathbf{H}_{2}-\mathrm{NLQ}_{2+}$, and Me-Q12+(isovalue: 0.002). 


\section{Electron Paramagnetic Resonance Spectroscopy}

Electron paramagnetic resonance (EPR) spectroscopy was conducted on a Bruker ELEXSYS II E500 with microwave frequency of $c a .9 .38 \mathrm{GHz}$ at $288 \mathrm{~K} .1 \mathrm{~mm}$ ID sample tubes were used for solutions and $4 \mathrm{~mm}$ ID sample tubes were used for solids. The spectra were acquired at $1 \mathrm{G}$ modulation and $100 \mathrm{kHz}$ modulation frequency with various powers.

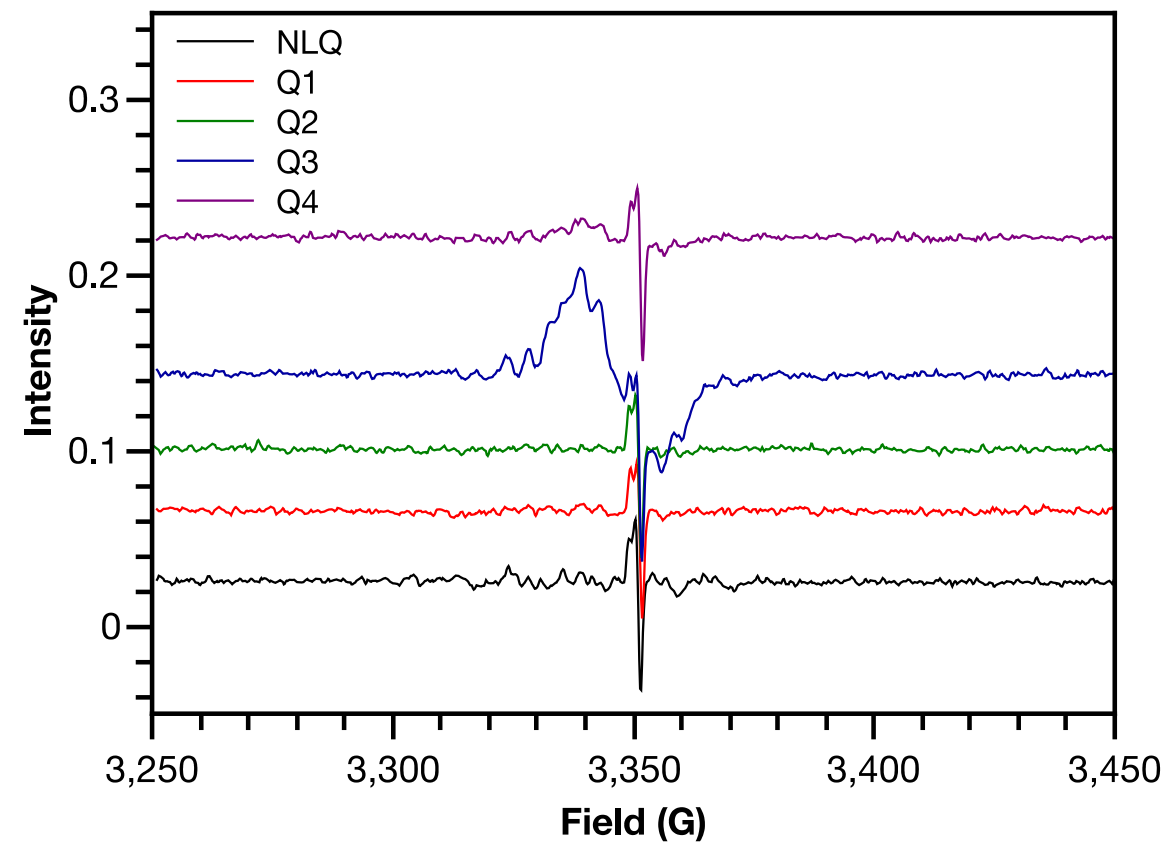

Figure S13. EPR spectra of NLQ and Qn in dichloromethane solution with triethylamine $(\mathrm{v} / \mathrm{v}=$ 10/1). Spin concentration was calculated as ca. 10-4 10-3 spin / molecule. 


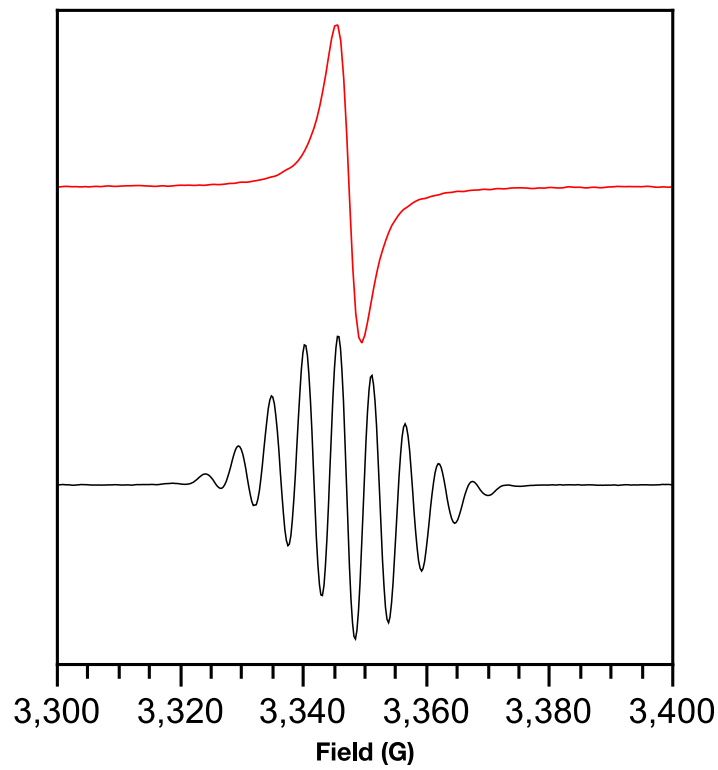

Figure S14. EPR spectra of Me-Q12+ $2 \mathrm{SbCl}_{6}$ in solid state (red) and in dichloromethane solution (black) at room temperature.

\section{SQUID Measurement}

Magnetic measurements were conducted on a Quantum Design MPMS XL SQUID magnetometer from 2 to $300 \mathrm{~K}$. The magnetization measurements were performed at $2 \mathrm{~K}$ over the magnetic field range of 250-70000 Oe. The diamagnetic contributions of the sample holders and the atoms were corrected with blank holders and the Pascal's constants.

NLQ fit well with theoretical doublet plot, showing no spin-spin coupling. The data spots of Q1 and Q2 were fit based on singlet ground state and triplet excited state. For Q3, the ground state changes from singlet to triplet with a small positive $J$ value. More interestingly, the fitting for Q4 could not be achieved using a singlet-triplet model as the M/Msat curve of Q4 is above the theoretical $\mathrm{M} / \mathrm{M}$ sat curve for a triplet $(S=1)$, which indicating higher spin ground state could be involved. The $J$ values shift from small negative to positive as the conjugation systems are enlarged from $\mathbf{Q 1}$ to $\mathbf{Q 4}$. The antiferromagnetically coupled nature of radical pair(s) in $\mathbf{Q 1}$ and $\mathbf{Q 2}$ is 
consistent with behaviors of benzene bridged diradicals with 1,4-connection.2 The ferromagnetic coupling in Q3 and Q4 is likely caused by the nearly degenerated nature of radical molecular orbitals in solid state local enviroments.3,4
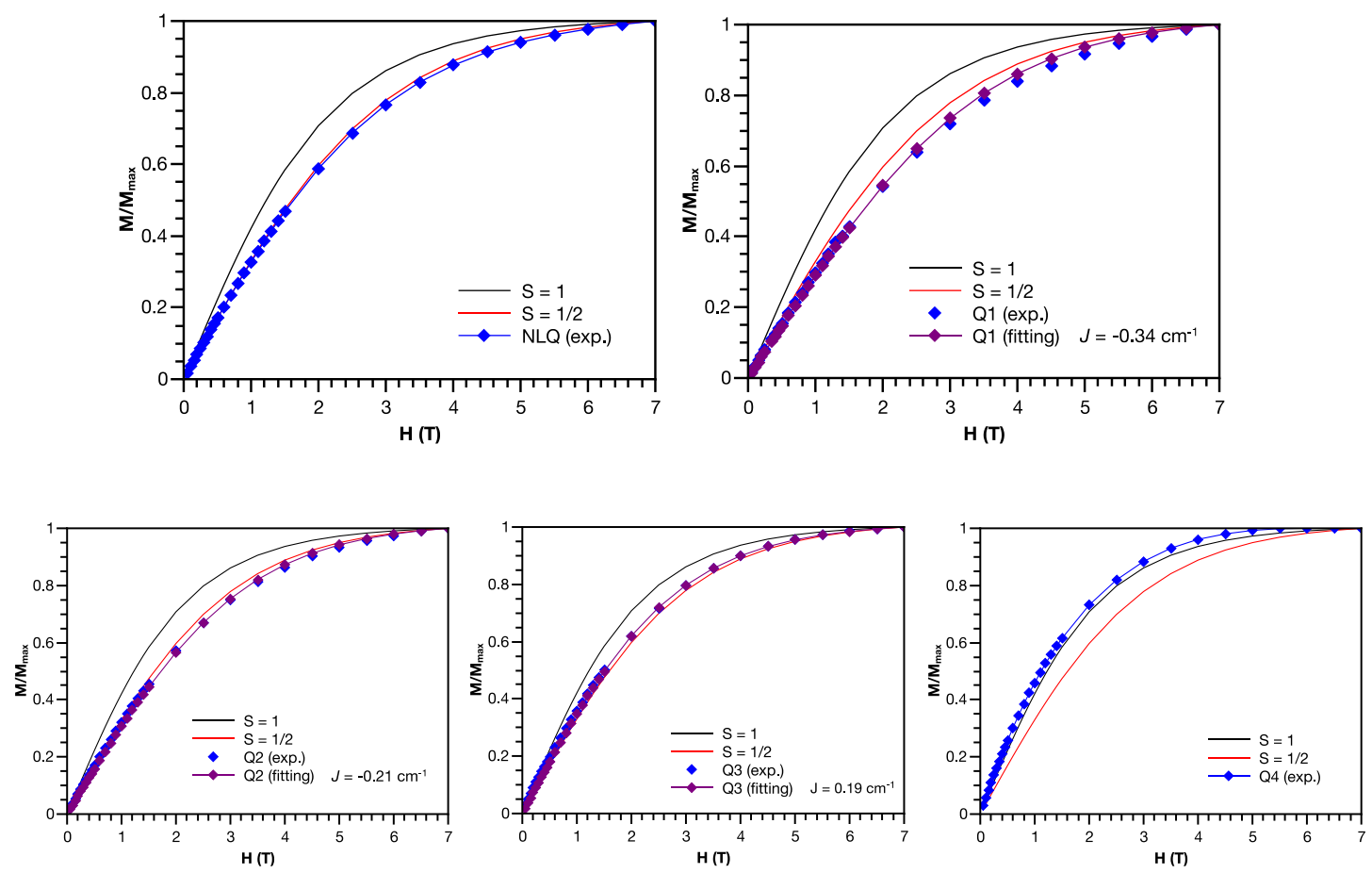

Figure S15. Magnetization saturation plots of NLQ and Qn with PTSA (5n equiv.) at 2 K (Dots: experimental data points; Lines: fitting curves).

\section{Conductivity Measurement}

Conductivity of Q1 and PTSA treated Q1 and Q2 was evaluated by a four-point probe method under vacuum. Pellet samples were prepared by compressing the powder materials $(\sim 0.1 \mathrm{~g})$ in a mold with inner diameter of $25 \mathrm{~mm}$ at pressure of $20 \mathrm{kPa}$ in air. The electrical resistance was measured with a Keithley 2450 SourceMeter. The thickness of the pellet was measured using scanning electron microscopy as $c a \cdot 10-2 \mathrm{~cm}$. The electrical conductivity was calculated based on the slope of the I-V plots. Neutral Q1 exhibited no observable conductivity. (Figure S16c) In 
contrast, the protonated Q1 and Q2 showed conductivity of $9.2 \times 10-6$ and $6.4 \times 10-6 \mathrm{~S} / \mathrm{cm}$, respectively (Figure S16a, b). The mixture of Me-Q12+ and PTSA was also measured under the same condition, showing no obvious conductivity (Figure S16d), indicating the electronic conductivity was related to the protonated pernigraniline moieties.
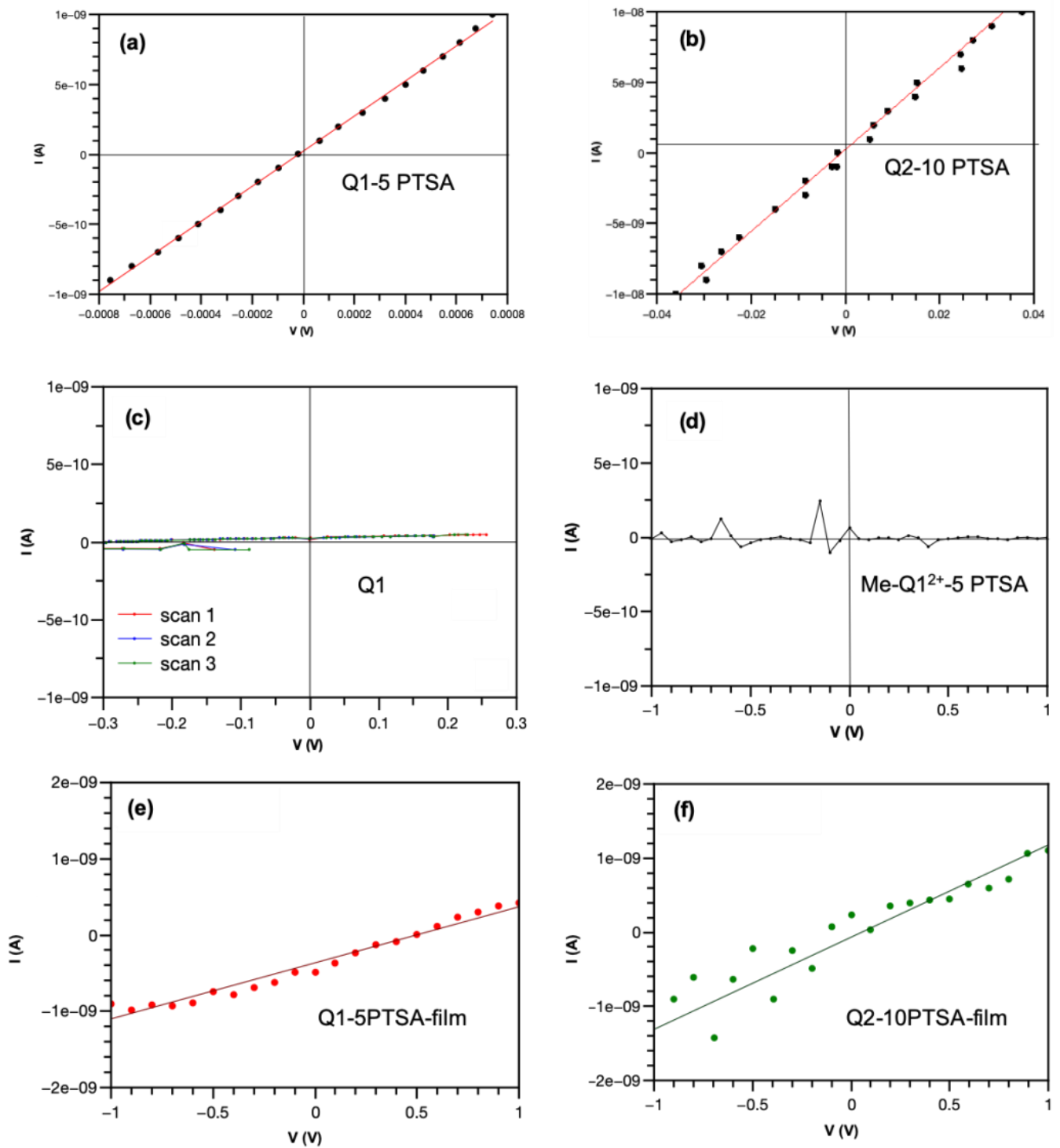

Figure S16. 4-probe conductivity measurement of (a) Q1-5PTSA, (b) Q2-10PTSA, (c) pristine Q1, (d) Me-Q12+ mixed with 5 equiv. PTSA as pellet samples, and (e) Q1-PTSA and (f) Q210PTSA as drop-casted films. 
AC impedance experiment was performed with a Solartron SI 1287 electrochemical interface and a Solartron SI 1260 impedance/gain-phase analyzer. The AC amplitude was set as $10 \mathrm{mV}$ versus open circuit. The frequency sweep range was $0.01 \mathrm{~Hz}$ to $100 \mathrm{kHz}$. Gold paste was applied on both sides of a Q1-5PTSA pellet. The measured values of cross-sectional area (A) and thickness (d) were $0.35 \mathrm{~cm} 2$ and $0.08 \mathrm{~cm}$. The impedance spectrum showed only one semicircle Nyquist plot, 5 which should be attributed to the electronic conductive component as charge transport occurs through hopping. The real part of the impedance was attributed to the hopping process and the imaginary contribution came from the atomic polarizability.6, 7
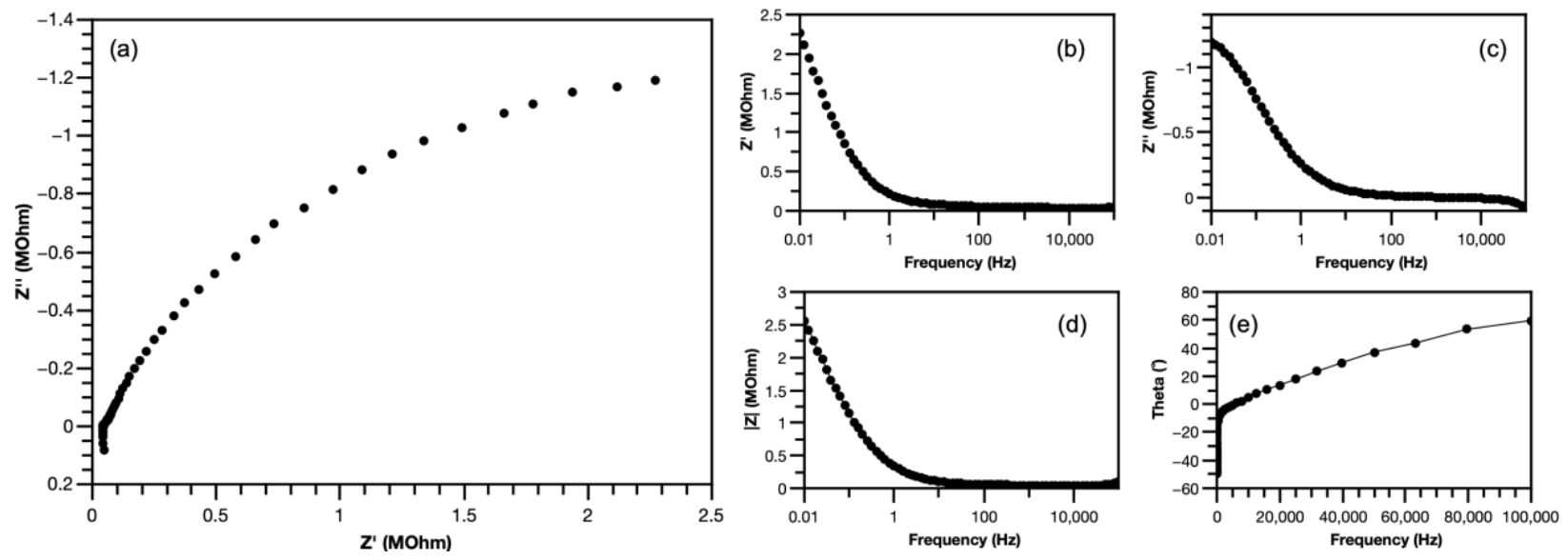

Figure S17. (a)Impedance plot and frequency dependence of (b)(c)(d) impedance and (e)phase shift of Q1-5PTSA pellet. Frequency was swept from $0.01 \mathrm{~Hz}$ to $100 \mathrm{kHz}$.

\section{Thermogravimetric Analysis}

TGA was undertaken with a TA Q500 thermogravimetric analyzer in nitrogen flow at $60.0 \mathrm{ml} / \mathrm{min}$ at a heating rate of $10.00{ }^{\circ} \mathrm{C} / \mathrm{min}$, showing much higher residue weight at $900{ }^{\circ} \mathrm{C}$ for Q1 Q3 compared to the $0 \%$ residue of NLQ. 


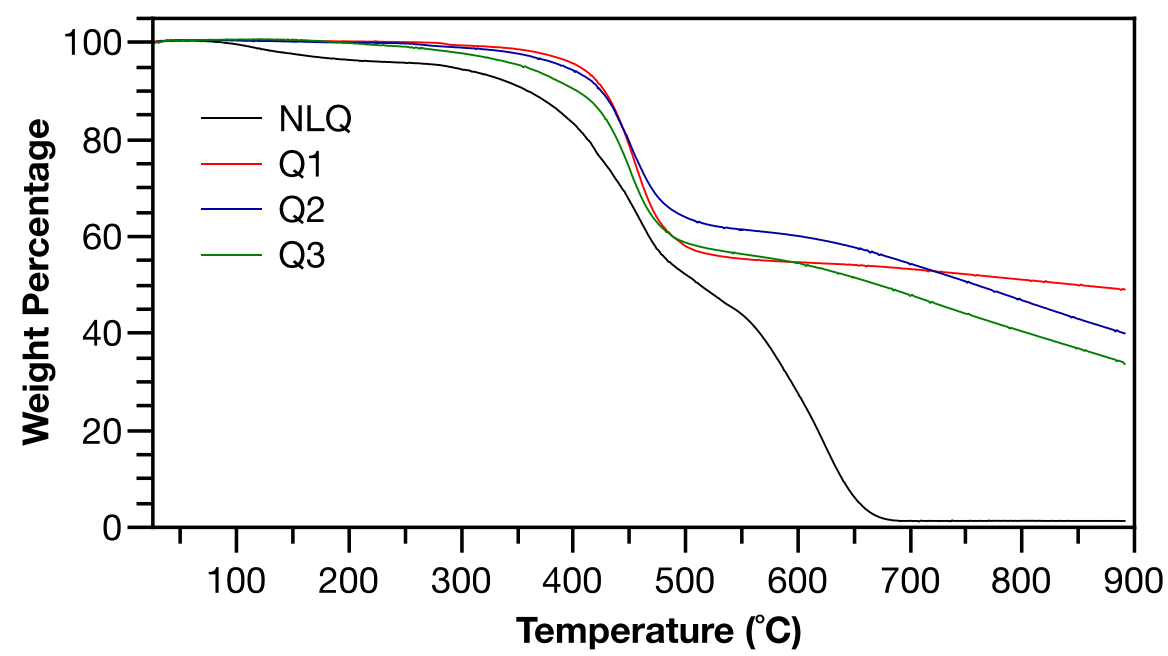

Figure S18. TGA plots of NLQ and Qn in nitrogen.

\section{X-ray Crystallography}

A Leica MZ 75 microscope was used to identify a representative sample of crystals of the same habit. The crystal mounted on a nylon loop was then placed in a cold nitrogen stream (Oxford) maintained at $100 \mathrm{~K}$. A BRUKER Venture X-ray (kappa geometry) diffractometer was employed for crystal screening, unit cell determination, and data collection. The goniometer was controlled using the APEX3 software suite. The sample was optically centered with the aid of a video camera such that no translations were observed as the crystal was rotated through all positions. The X-ray radiation employed was generated from a $\mathrm{Cu}-\mathrm{I} \mu \mathrm{s}$ X-ray tube $\left(\mathrm{K}_{\alpha}=1.5418 \AA ̊\right.$ with a potential of 50 $\mathrm{kV}$ and a current of $1.0 \mathrm{~mA}$ ). 45 data frames were taken at widths of $1^{\circ}$. These reflections were used to determine the unit cell. The unit cell was verified by examination of the $h k l$ overlays on several frames of data. No super-cell or erroneous reflections were observed. After careful examination of the unit cell, an extended data collection procedure (30 sets) was initiated using omega and phi scans. Integrated intensity information for each reflection was obtained by reduction of the data frames with the program APEX3. The integration method employed a three- 
dimensional profiling algorithm and all data were corrected for Lorentz and polarization factors, as well as for crystal decay effects. Finally, the data was merged and scaled to produce a suitable data set. The absorption correction program SADABS was employed to correct the data for absorption effects. A solution was obtained readily $\left(Z=1 ; Z^{\prime}=0.5\right)$ using XT/XS in APEX3. Hydrogen atoms were placed in idealized positions and were set riding on the respective parent atoms. All non-hydrogen atoms were refined with anisotropic thermal parameters. Appropriate restraints and constraints were used to keep the bond distances, angles and thermal ellipsoids meaningful. Absence of additional symmetry or void were confirmed using PLATON (ADDSYM). The structure was refined (weighted least squares refinement on $F_{2}$ ) to convergence. Olex2 was employed for the final data presentation and structure plots.

\section{Table S5. Crystal data and structure refinement for Q1.}

Empirical formula

Formula weight

Temperature

Wavelength

Crystal system

Space group

Unit cell dimensions

Volume

Z

Density (calculated)
$\mathrm{C} 62 \mathrm{H} 78 \mathrm{~N} 2$

851.26

$100.0 \mathrm{~K}$

$0.71073 \AA$

Monoclinic

P $121 / \mathrm{c} 1$

$$
\begin{array}{ll}
\mathrm{a}=20.9363(13) \AA & \alpha=90^{\circ} . \\
\mathrm{b}=10.6422(7) \AA & \beta=98.087(2)^{\circ} . \\
\mathrm{c}=11.1562(7) \AA & \gamma=90^{\circ} .
\end{array}
$$

2461.0(3) $\AA^{3}$

2

$1.149 \mathrm{Mg} / \mathrm{m}^{3}$

S33 

Absorption coefficient
$0.065 \mathrm{~mm}^{-1}$
$\mathrm{F}(000)$
928
Crystal size
$0.681 \times 0.204 \times 0.042 \mathrm{~mm}^{3}$
Theta range for data collection
2.658 to $19.192^{\circ}$.
Index ranges
$-19<=\mathrm{h}<=19,-9<=\mathrm{k}<=9,-10<=1<=10$
Reflections collected
41972
Independent reflections
$2040[\mathrm{R}(\mathrm{int})=0.0865]$
Completeness to theta $=19.192^{\circ}$
$99.7 \%$
Absorption correction
Semi-empirical from equivalents
Max. and min. transmission
0.7443 and 0.5694
Refinement method
Full-matrix least-squares on $\mathrm{F}^{2}$
Data / restraints / parameters
$2040 / 0 / 293$
Goodness-of-fit on $\mathrm{F}^{2}$
1.141
Final $\mathrm{R}$ indices [I $>2 \operatorname{sigma}(\mathrm{I})]$
$\mathrm{R} 1=0.0691, \mathrm{wR} 2=0.1573$
$\mathrm{R}$ indices (all data)
$\mathrm{R} 1=0.0826, \mathrm{wR} 2=0.1689$
Extinction coefficient
$\mathrm{n} / \mathrm{a}$
Largest diff. peak and hole
0.414 and -0.247 e. $\AA^{-3}$

Table S5. Crystal data and structure refinement for Me-Q12+-2SbCl6-

Empirical formula

C66 H88 Cl16 N2 Sb2

Formula weight

1720.08

Temperature

$100.0 \mathrm{~K}$ 
Wavelength

Crystal system

Space group

Unit cell dimensions

Volume

Z

Density (calculated)

Absorption coefficient

$\mathrm{F}(000)$

Crystal size

Theta range for data collection

Index ranges

Reflections collected

Independent reflections

Completeness to theta $=67.679^{\circ}$

Absorption correction

Max. and min. transmission

Refinement method

Data / restraints / parameters

Goodness-of-fit on $\mathrm{F}^{2}$

Final R indices $[\mathrm{I}>2 \operatorname{sigma}(\mathrm{I})]$
$1.54178 \AA$

Triclinic

P-1

$\mathrm{a}=8.8952(3) \AA \quad \alpha=84.5548(12)^{\circ}$.

$\mathrm{b}=11.4019(4) \AA \quad \beta=89.4723(12)^{\circ}$.

$\mathrm{c}=20.5690(7) \AA \quad \gamma=67.0952(12)^{\circ}$.

$1.494 \mathrm{Mg} / \mathrm{m}^{3}$

$11.044 \mathrm{~mm}^{-1}$

872

$0.499 \times 0.128 \times 0.058 \mathrm{~mm}^{3}$

2.159 to $70.206^{\circ}$.

$-10<=\mathrm{h}<=9,-13<=\mathrm{k}<=13,-25<=\mathrm{l}<=25$

45841

$7214[\mathrm{R}(\mathrm{int})=0.0401]$

$99.4 \%$

Semi-empirical from equivalents

0.3841 and 0.1847

Full-matrix least-squares on $\mathrm{F}^{2}$

7214 / 139 / 456

1.095

$\mathrm{R} 1=0.0403, \mathrm{wR} 2=0.0960$

S35 
$\mathrm{R}$ indices (all data)

$\mathrm{R} 1=0.0436, \mathrm{wR} 2=0.0987$

Extinction coefficient

$\mathrm{n} / \mathrm{a}$

Largest diff. peak and hole 1.336 and -1.290 e. $\AA^{-}-3$
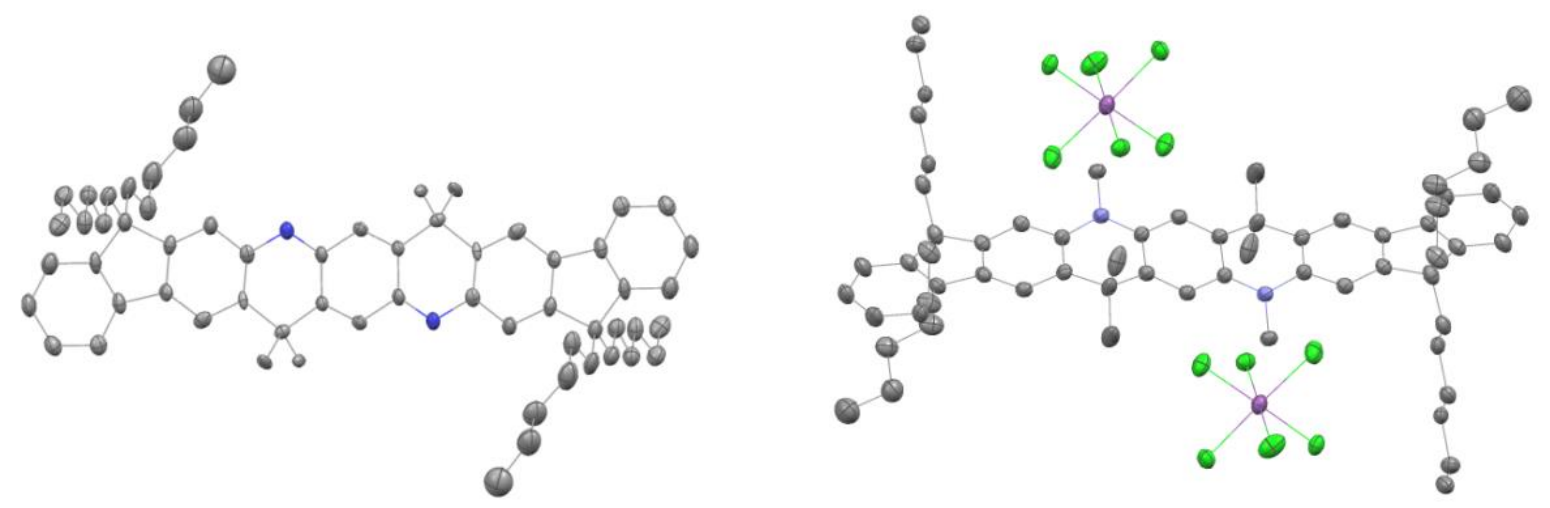

Figure S19. Single crystal structures of Q1 and Me-Q12+·2 SbCl6-.. Hydrogen atoms and solvent molecules were omitted for the sake of clarity. Thermal ellipsoids are scaled to the $50 \%$ probability level. 

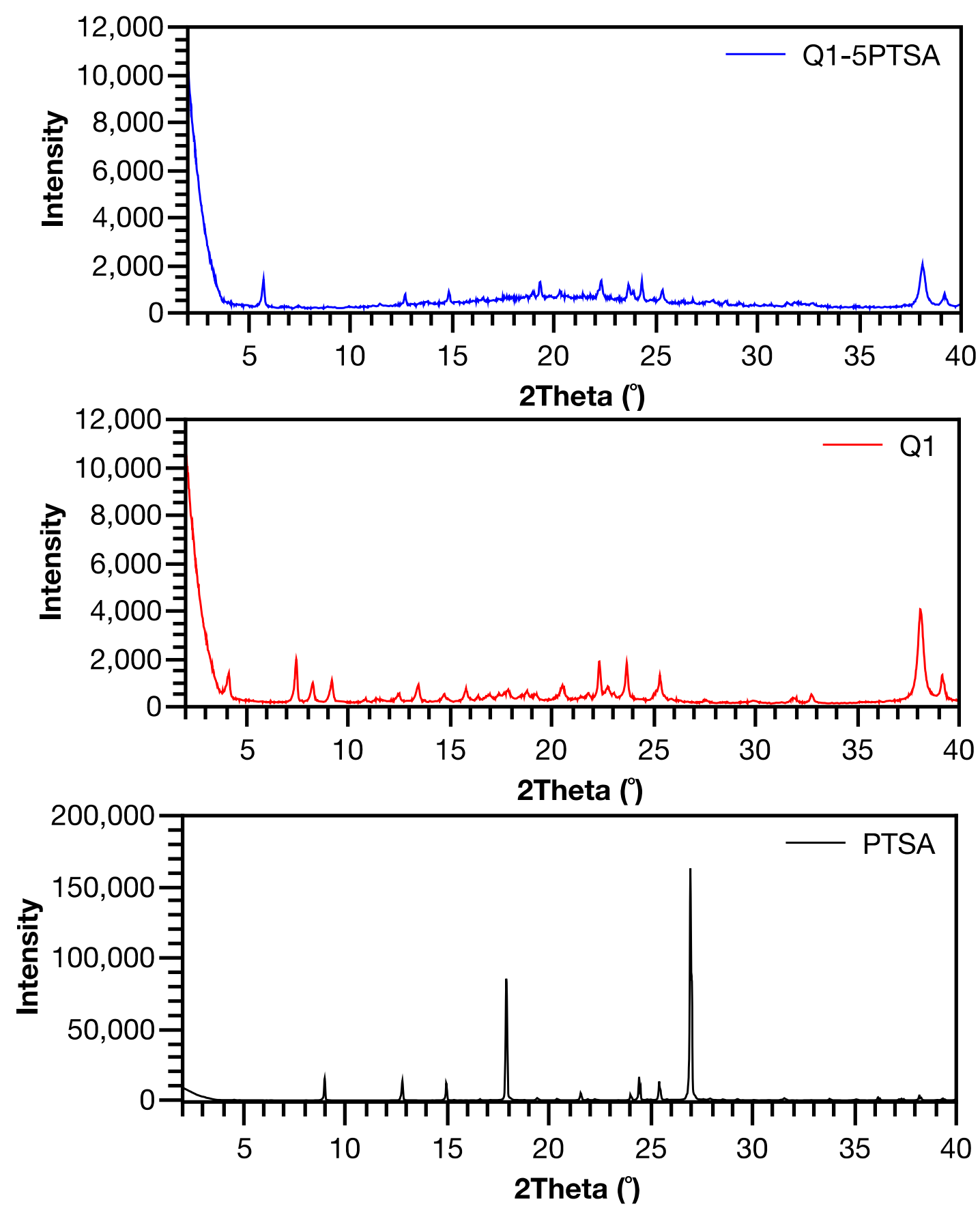

Figure S20. Powder X-ray Diffraction spectra of Q1, PTSA, and Q1 doped with 5 equiv. PTSA. 

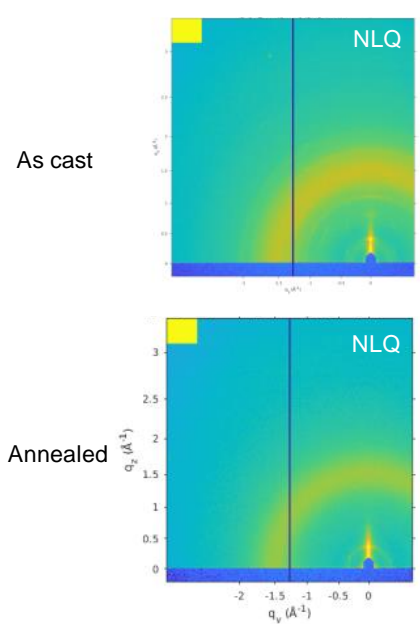
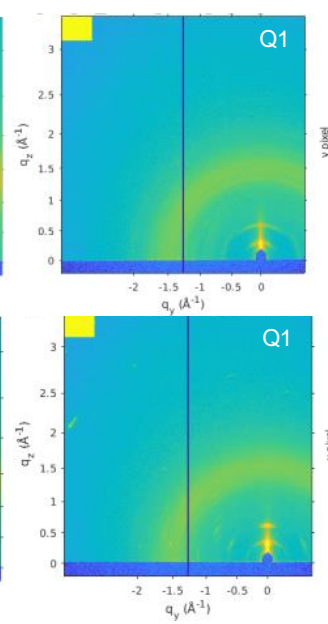
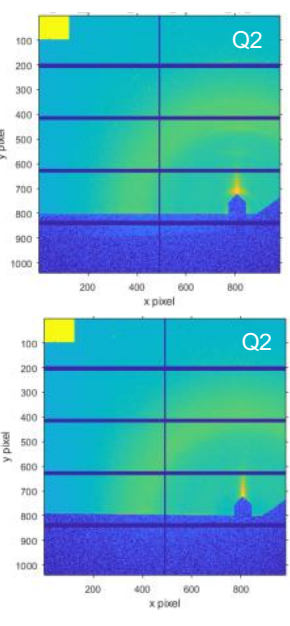
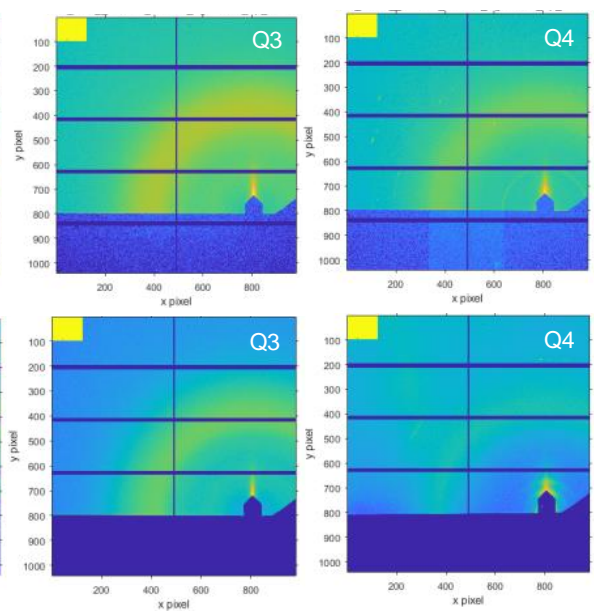

Figure S21. Grazing-incidence Wide-angle X-ray Scattering (GIWAXS) spectra of NLQ and Qn spin-coated films on silicon wafer before and after annealing. 


\section{H, $13 \mathrm{C}$ and NOESY NMR Spectra}
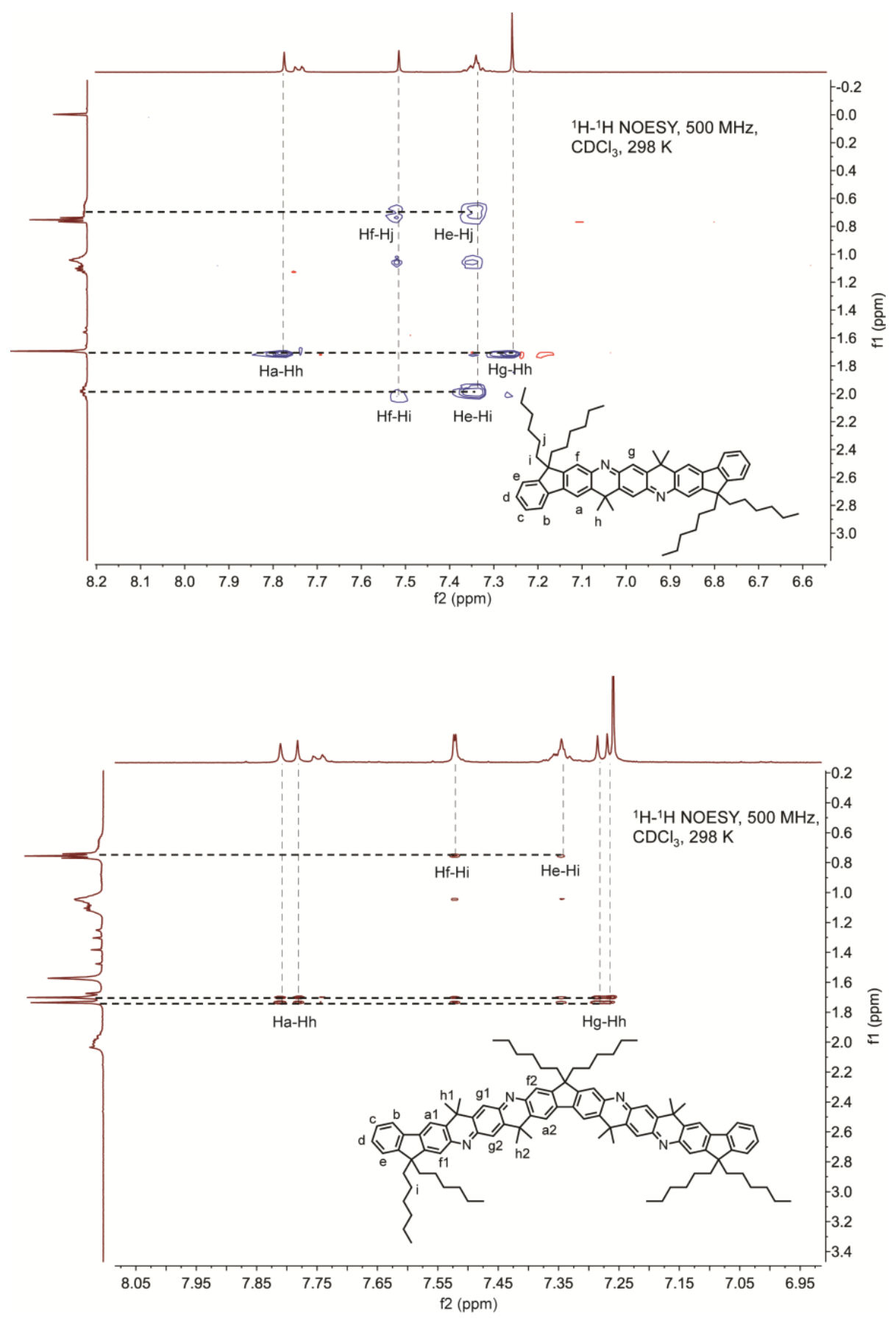

Figure S22. $1 \mathrm{H}-1 \mathrm{H}$ NOSEY NMR of Q1 and Q2 (500 MHz, CDCl3, RT). 


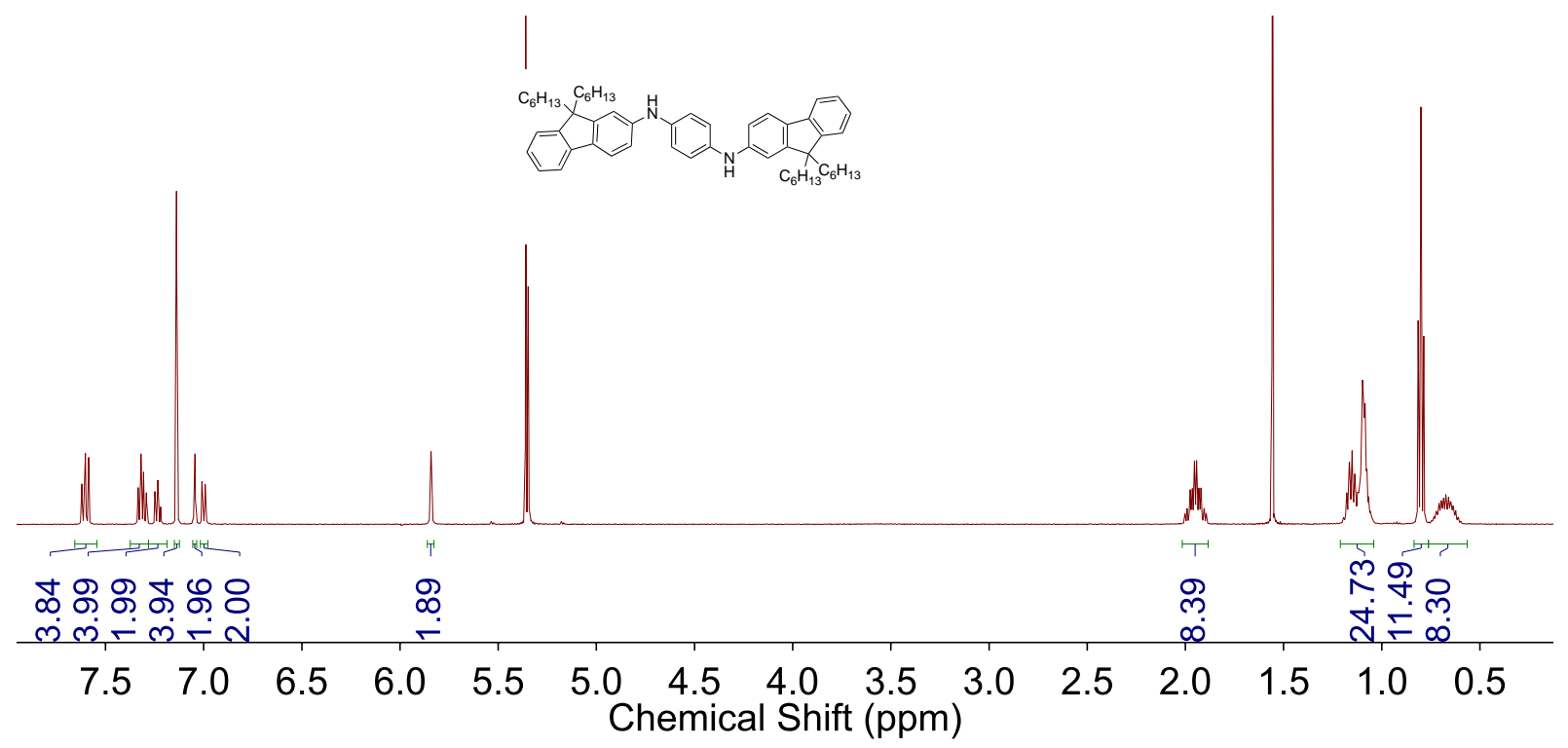

${ }_{1} \mathrm{H}$ NMR of NLA (500 MHz, $\left.\mathrm{CD}_{2} \mathrm{Cl}_{2}, \mathrm{RT}\right)$.

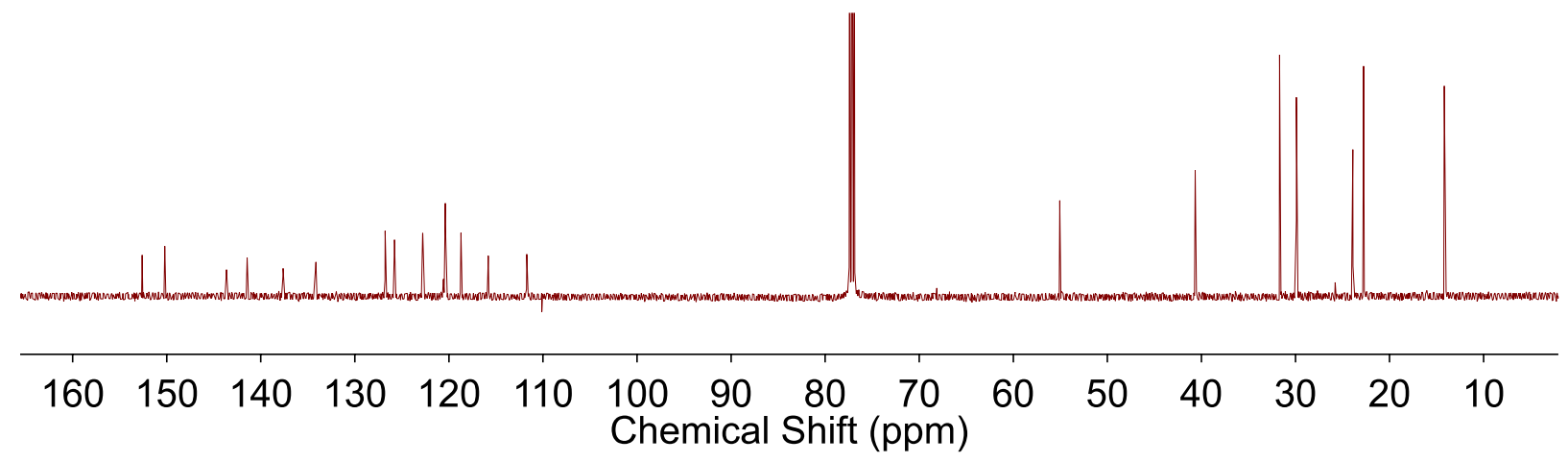

${ }_{13 \mathrm{C}} \mathrm{NMR}$ of NLA (125 MHz, $\left.\mathrm{CDCl}_{3}, \mathrm{RT}\right)$. 

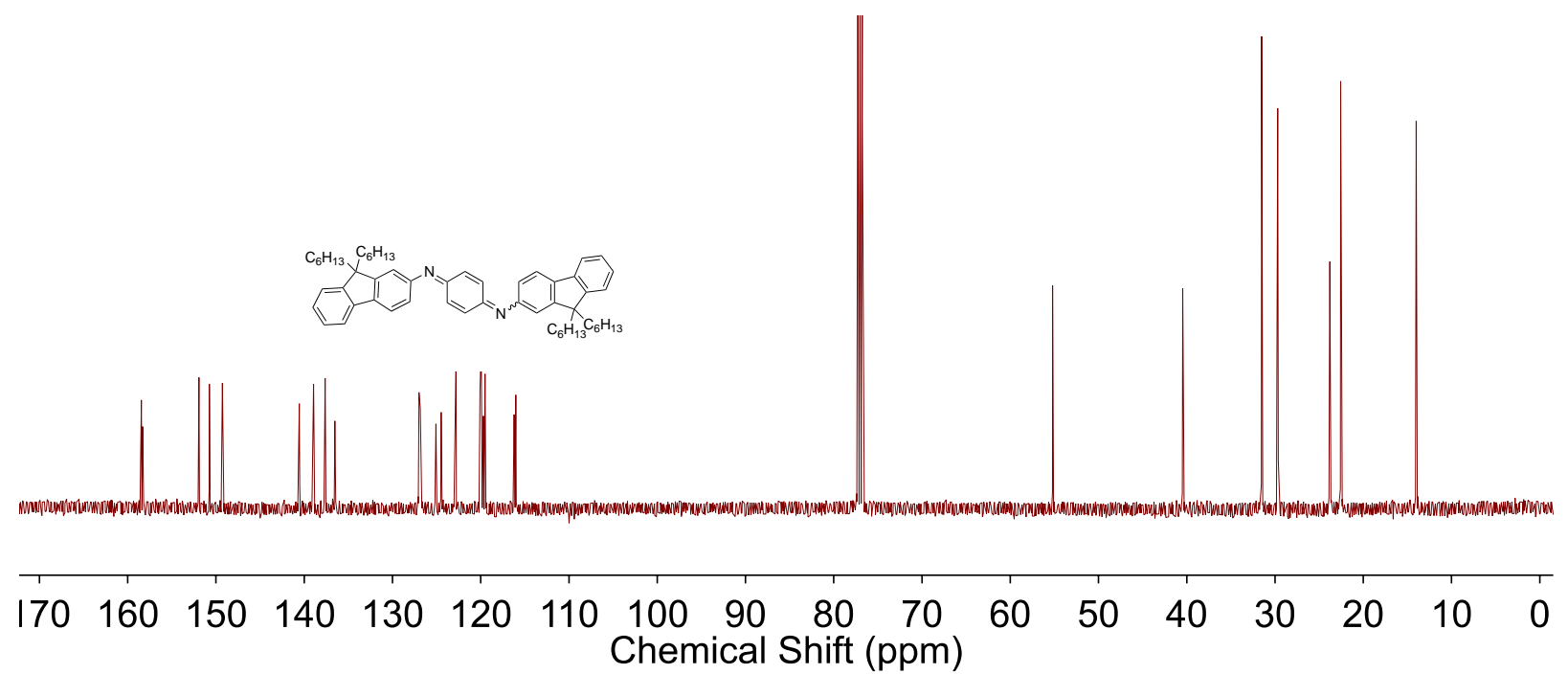
${ }_{13} \mathrm{C}$ NMR of NLQ (125 MHz, $\left.\mathrm{CDCl}_{3}, \mathrm{RT}\right)$.

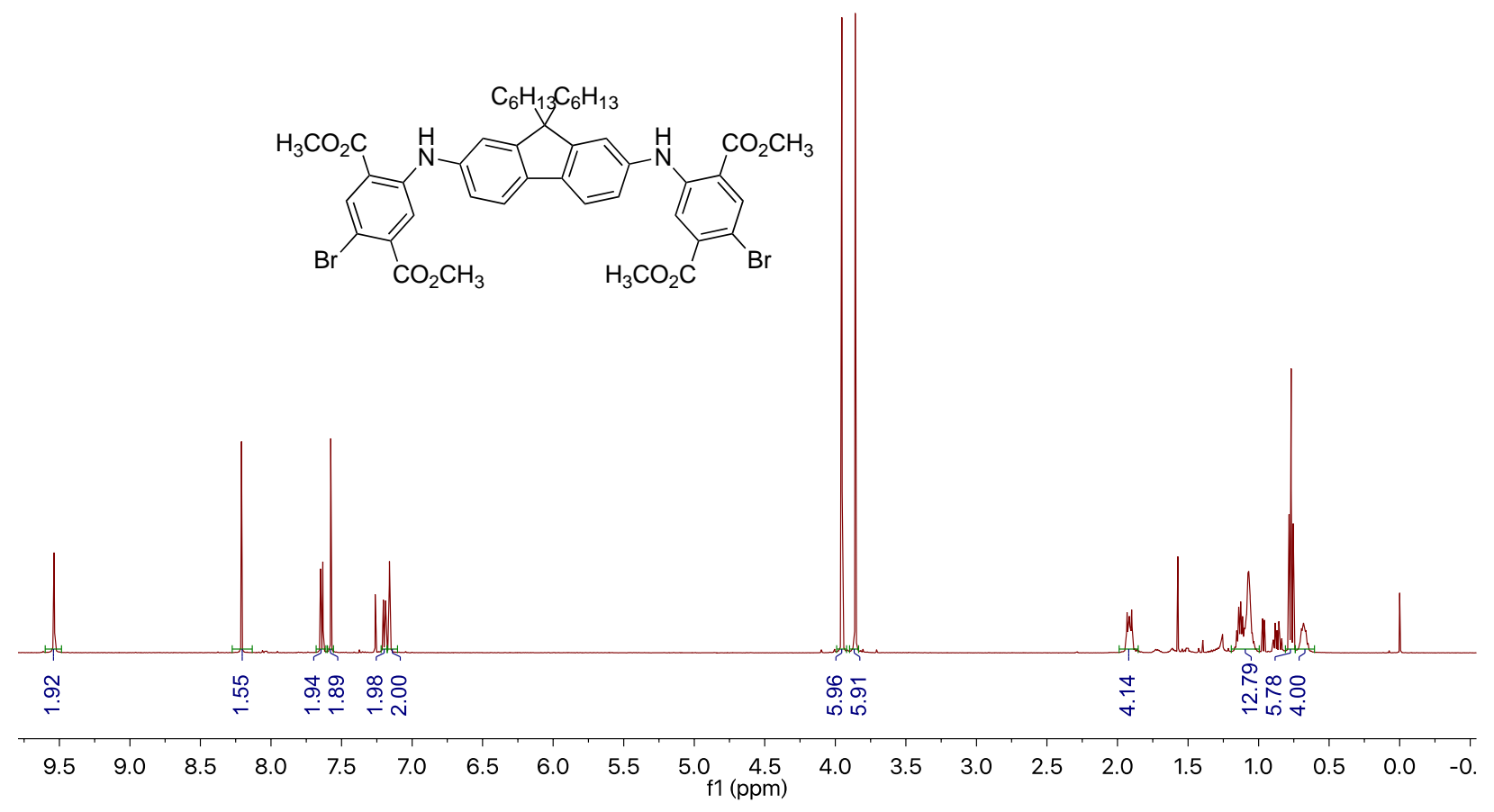

1H NMR of B2 (500 MHz, CDCl3, RT). 


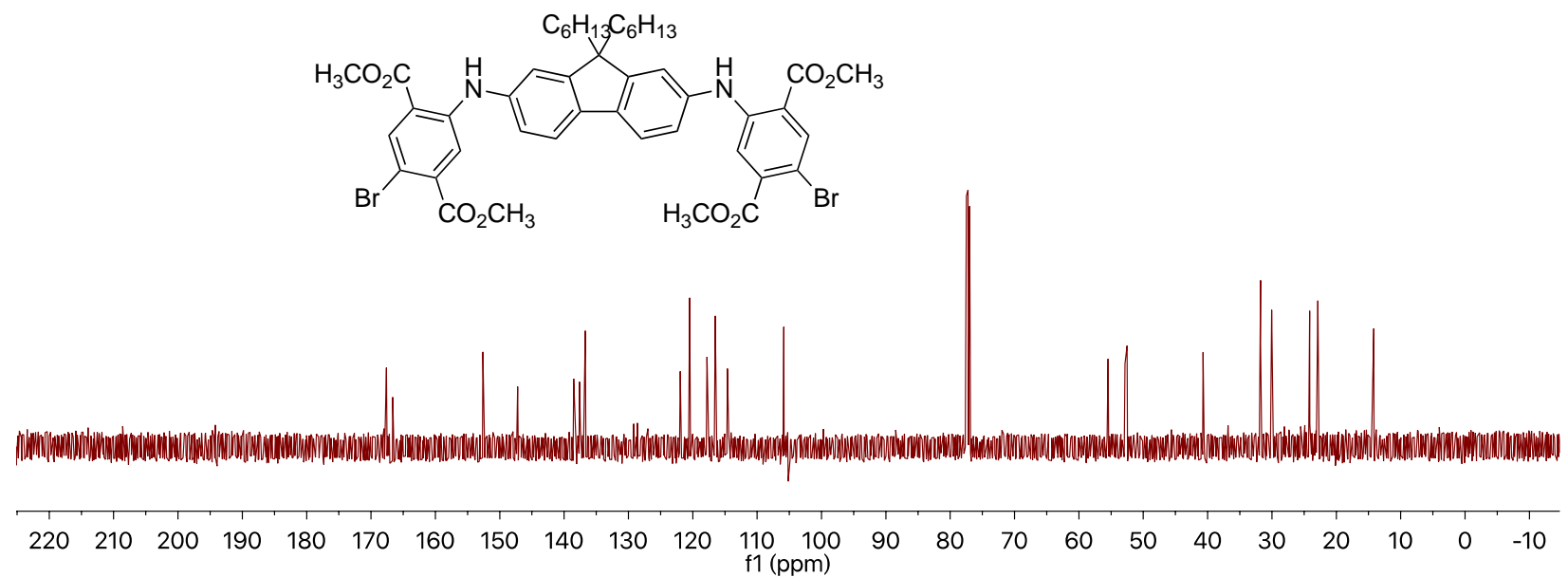

${ }_{13} \mathrm{C}$ NMR of B2 (125 MHz, $\left.\mathrm{CDCl}_{3}, \mathrm{RT}\right)$.

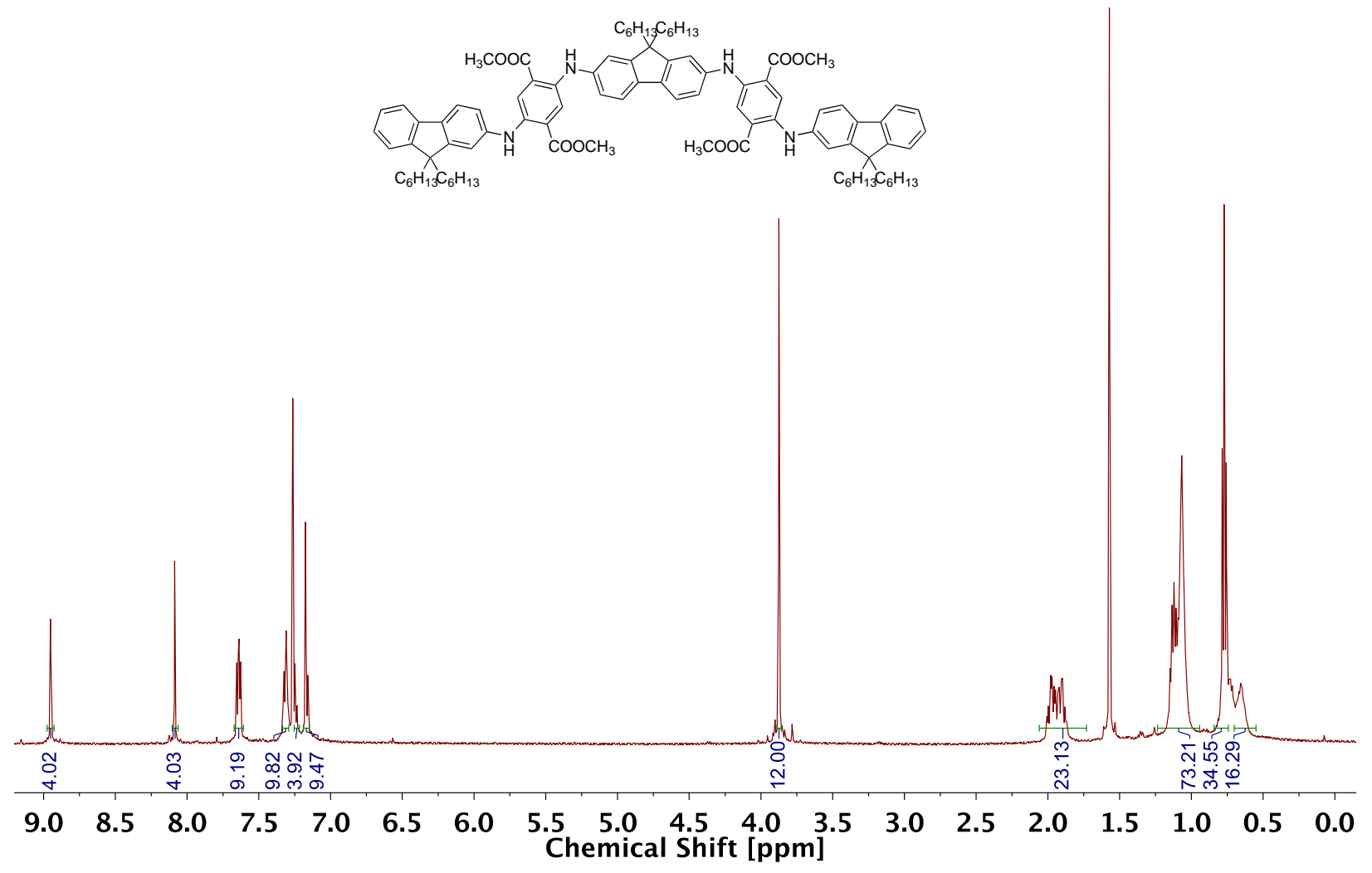

1H NMR of A2 (500 MHz, CDCl3, RT). 

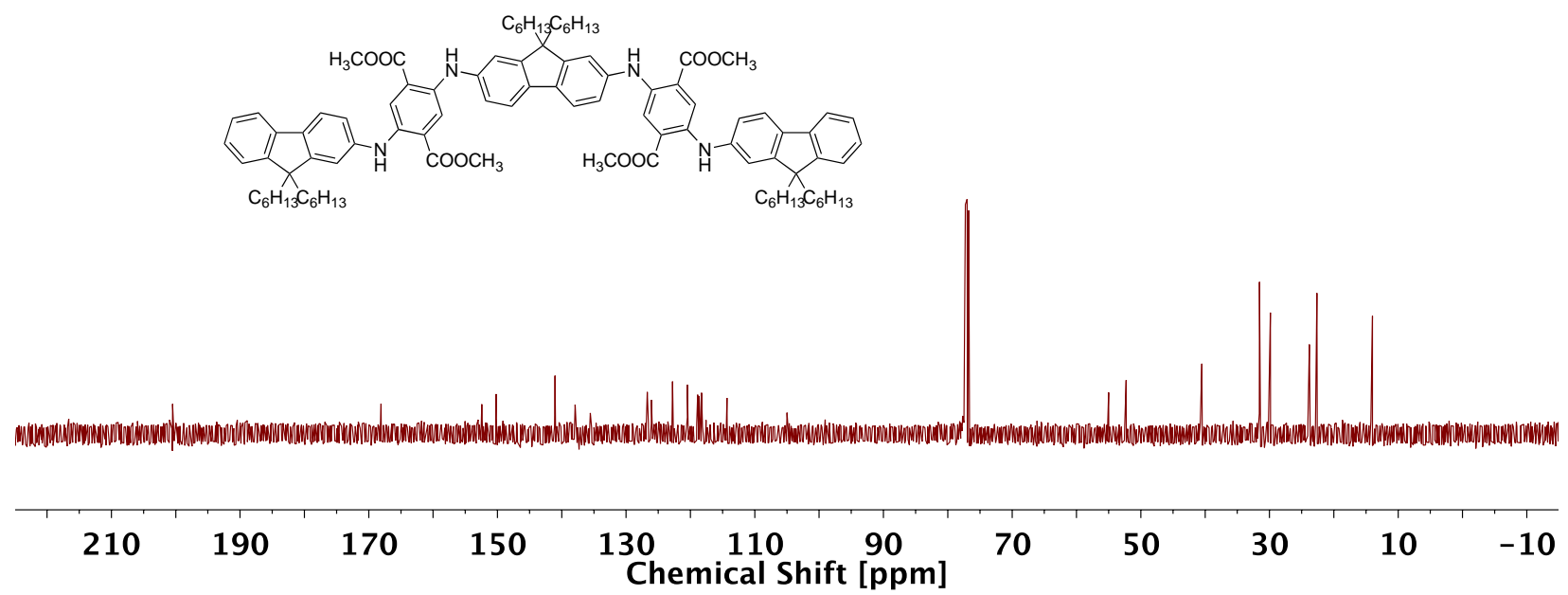
13C NMR of A2 (125 MHz, CDCl3, RT).

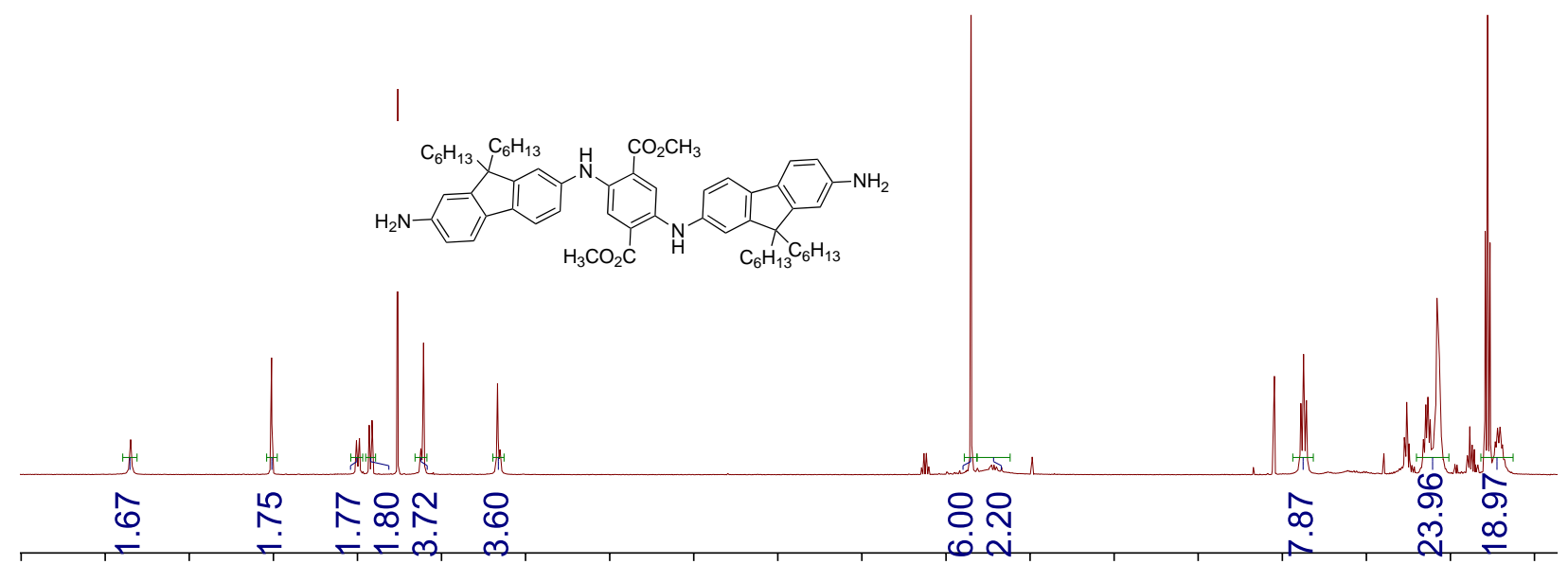

$\begin{array}{lllllllllllllllllll}.5 & 9.0 & 8.5 & 8.0 & 7.5 & 7.0 & 6.5 & 6.0 & 5.5 & 5.0 & 4.5 & 4.0 & 3.5 & 3.0 & 2.5 & 2.0 & 1.5 & 1.0 & 0.5\end{array}$ Chemical Shift (ppm)

1H NMR of Compound 6 (500 MHz, $\left.\mathrm{CDCl}_{3}, \mathrm{RT}\right)$.
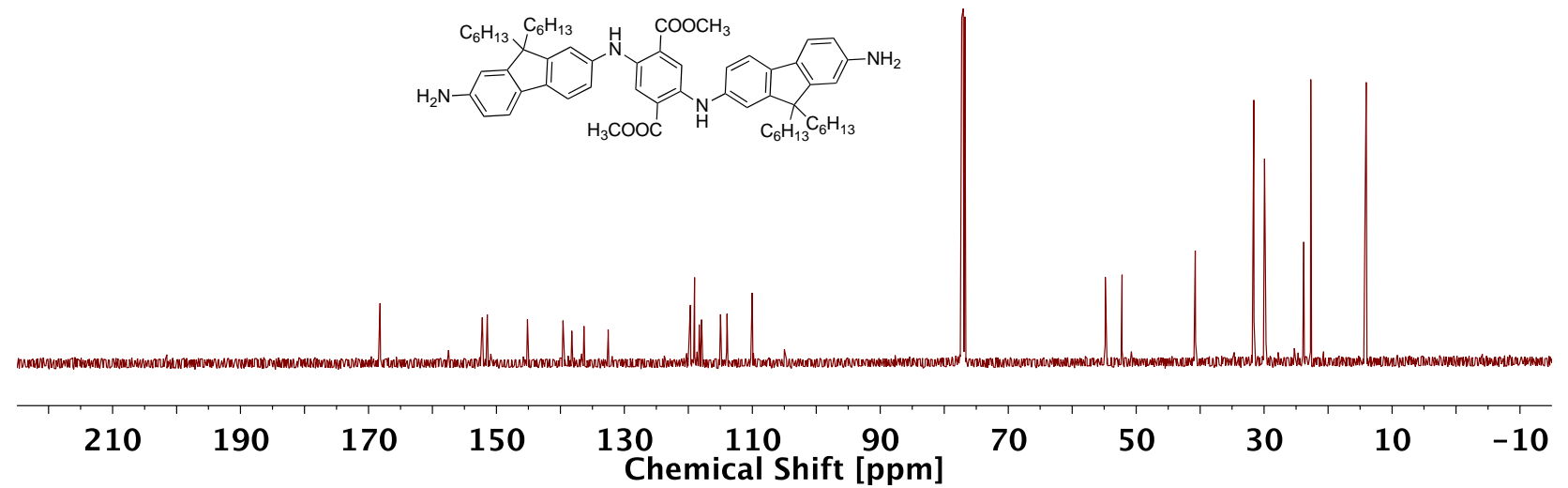

S43 
${ }_{13} \mathrm{C}$ NMR of Compound 6 (125 MHz, $\left.\mathrm{CDCl}_{3}, \mathrm{RT}\right)$.

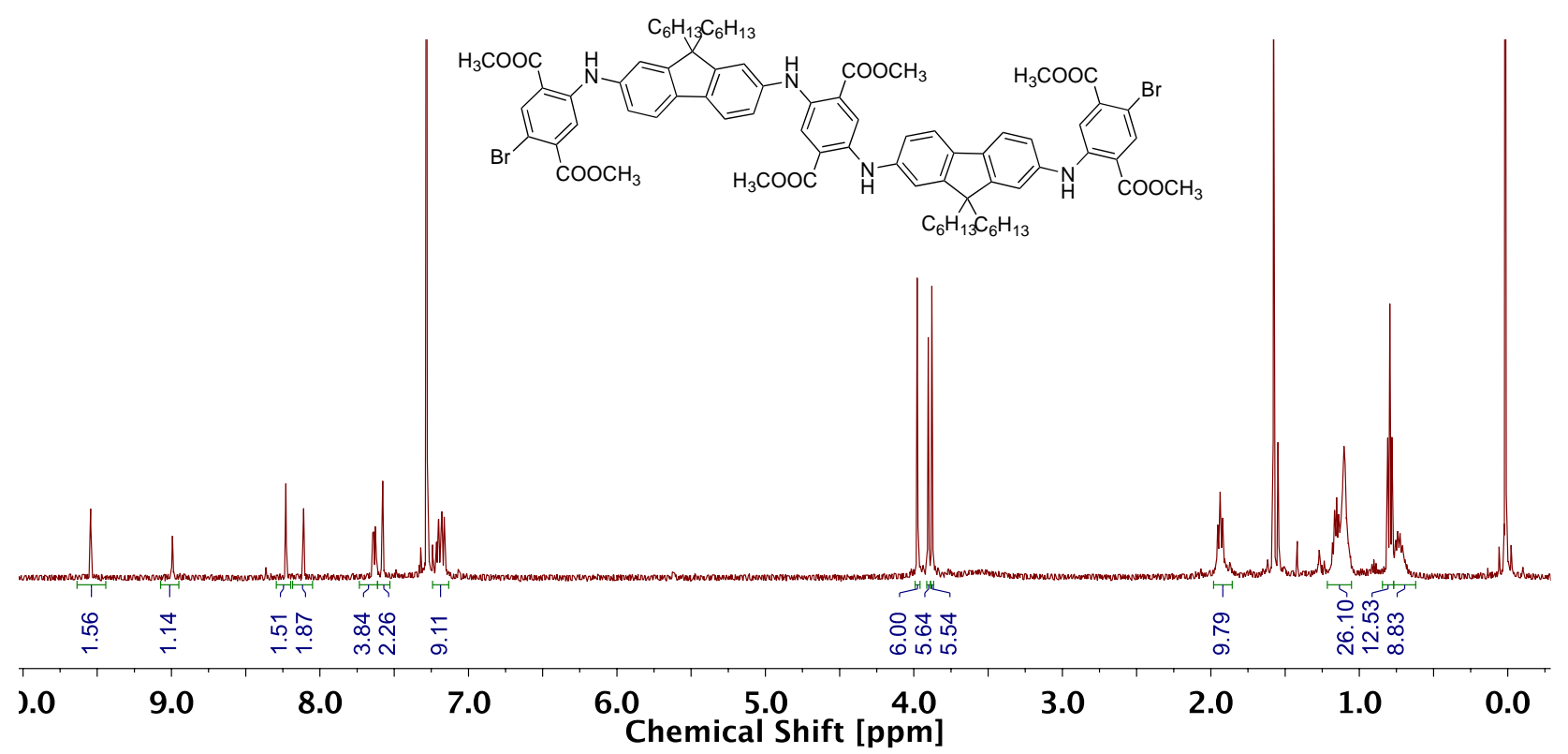

1H NMR of $\mathbf{B 3}\left(500 \mathrm{MHz}, \mathrm{CDCl}_{3}, \mathrm{RT}\right)$.
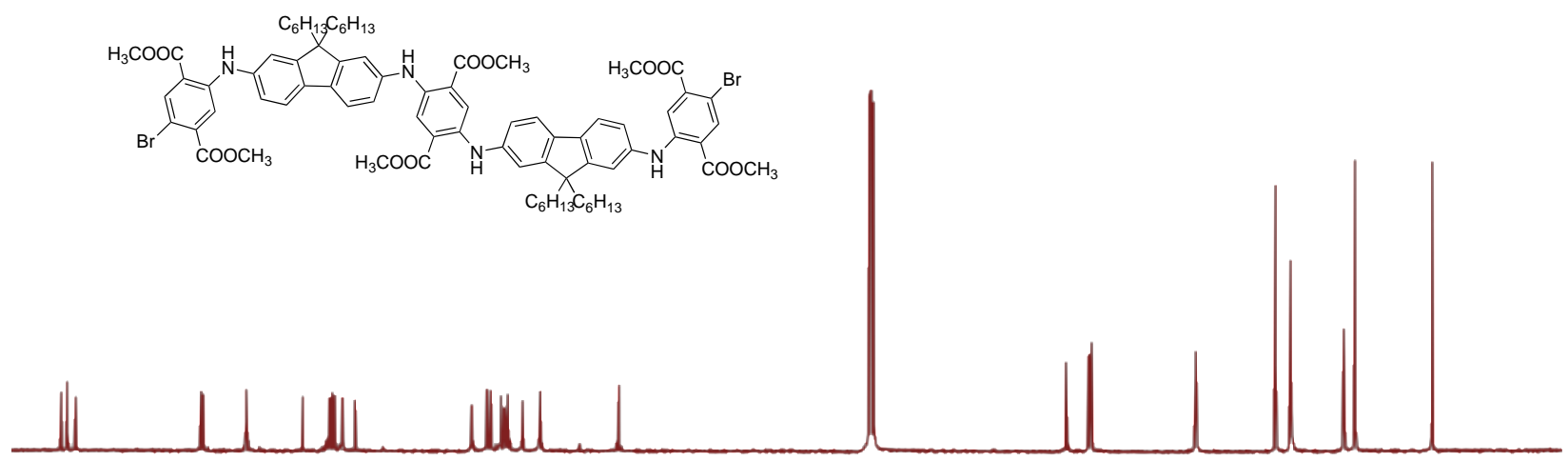

$\begin{array}{lllllllllllllllll}170 & 160 & 150 & 140 & 130 & 120 & 110 & \begin{array}{c}100 \\ \text { Chemical Shift (ppm) }\end{array} & \begin{array}{c}80 \\ \text { Che }\end{array} & 50 & 40 & 30 & 20 & 10 & 0\end{array}$ ${ }_{13 \mathrm{C}} \mathrm{NMR}$ of $\mathbf{B 3}\left(125 \mathrm{MHz}, \mathrm{CDCl}_{3}, \mathrm{RT}\right)$. 

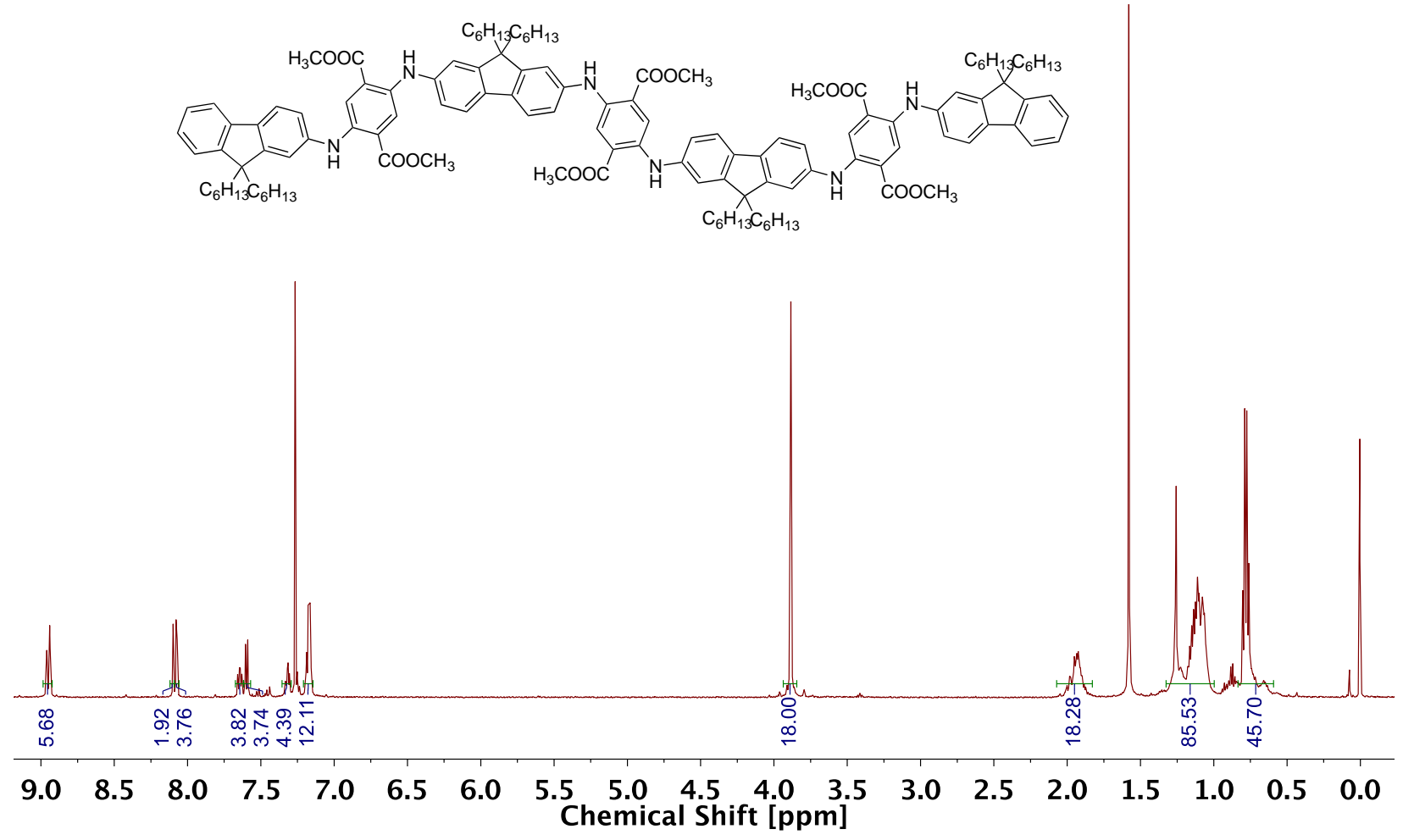

${ }_{1} \mathrm{H}$ NMR of $\mathbf{A 3}\left(500 \mathrm{MHz}, \mathrm{CDCl}_{3}, \mathrm{RT}\right)$.
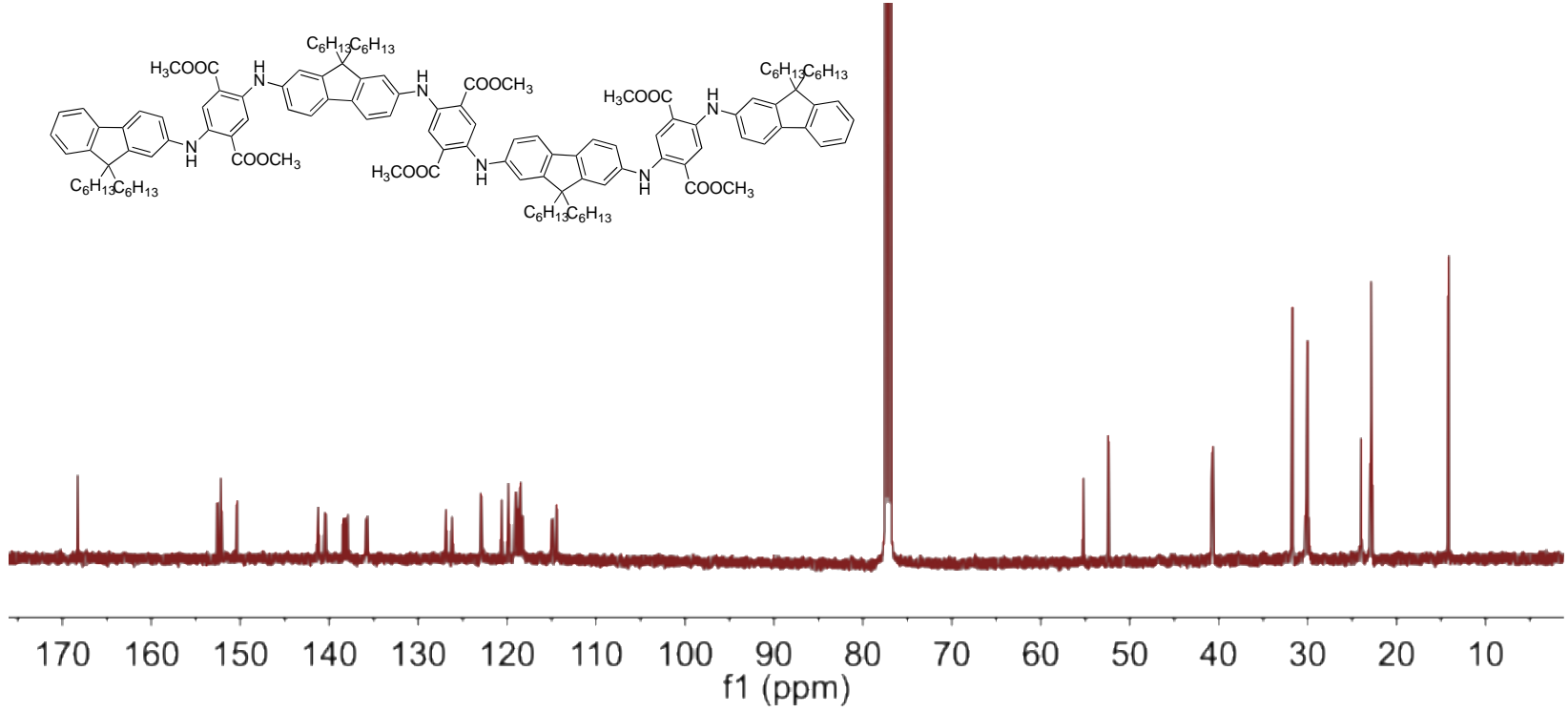

13 C NMR of $\mathbf{A 3}\left(125 \mathrm{MHz}, \mathrm{CDCl}_{3}, \mathrm{RT}\right)$. 


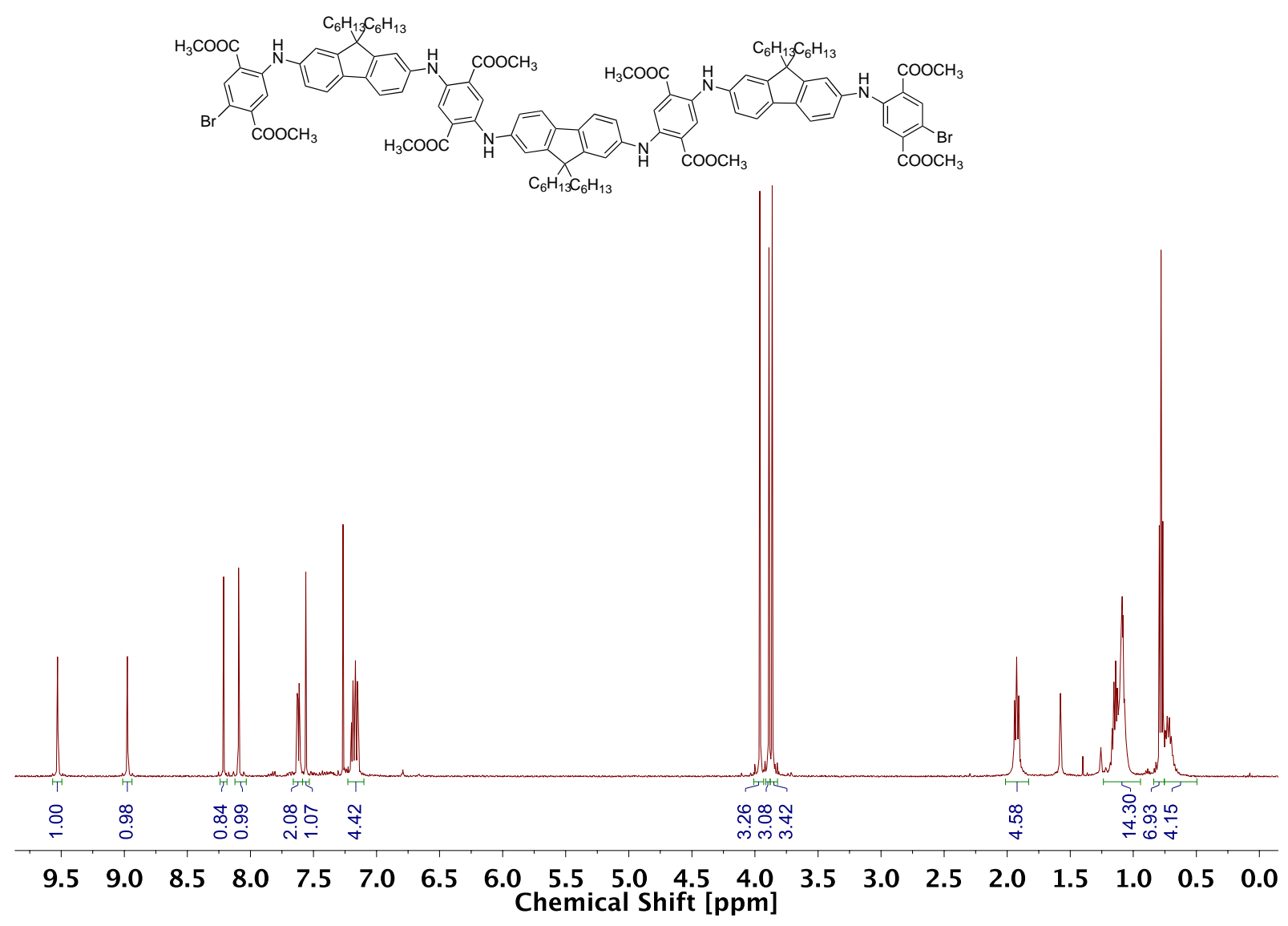

1H NMR of B4 (500 MHz, CDCl3, RT).

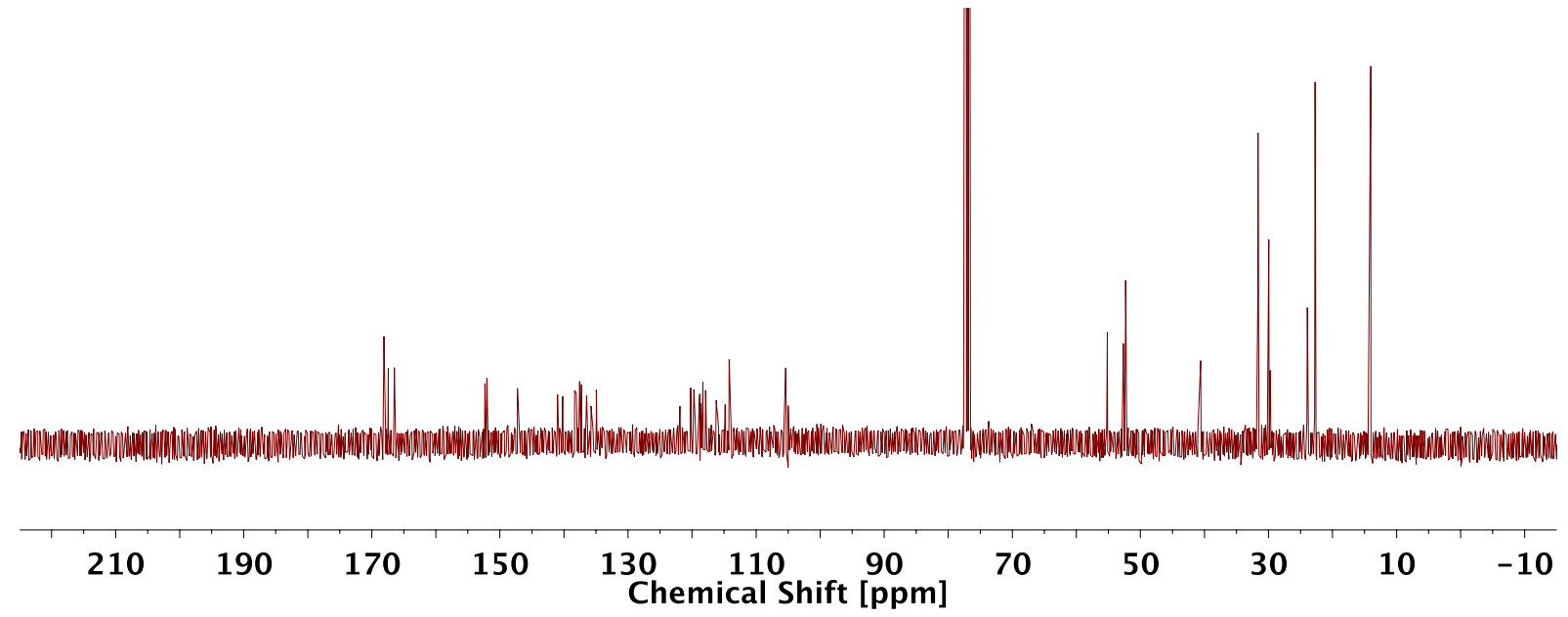

13C NMR of B4 (125 MHz, CDCl3, RT). 


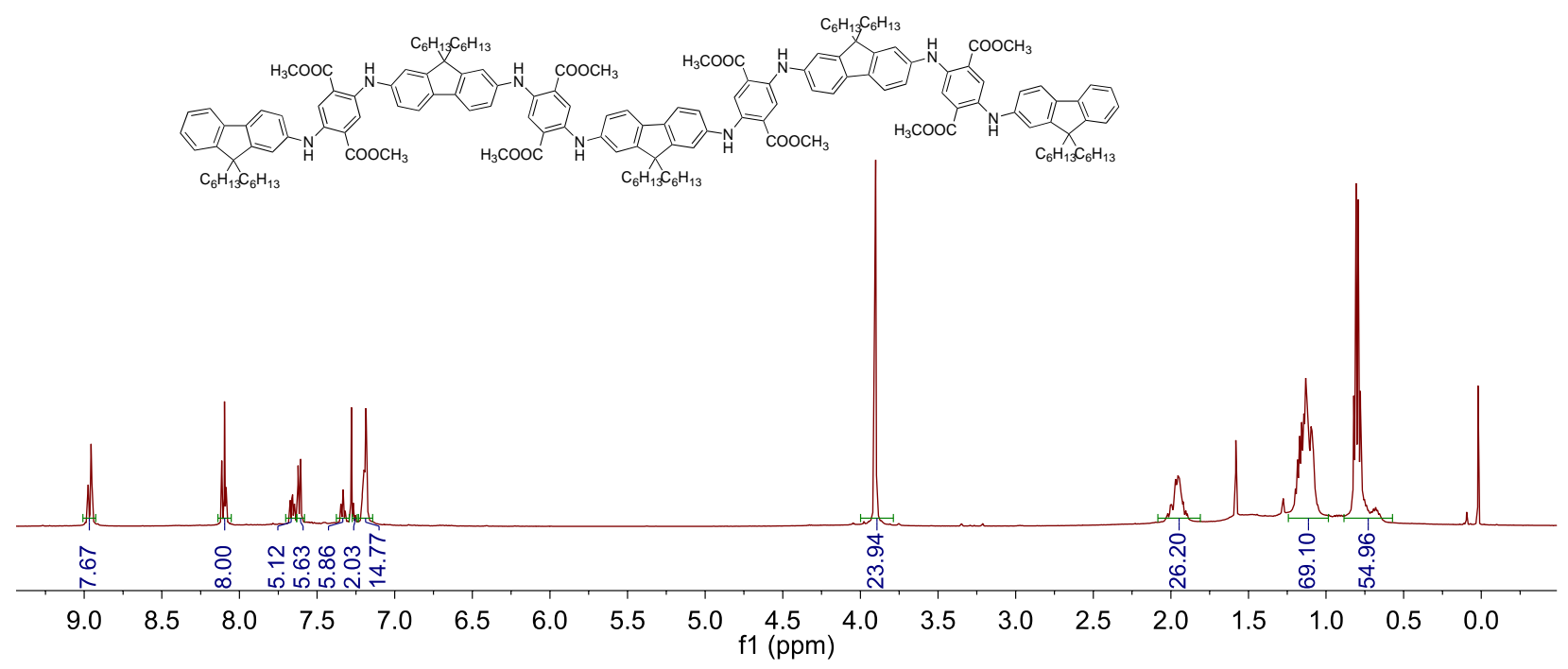

${ }_{1} \mathrm{H}$ NMR of A4 (500 MHz, CDCl3, RT).

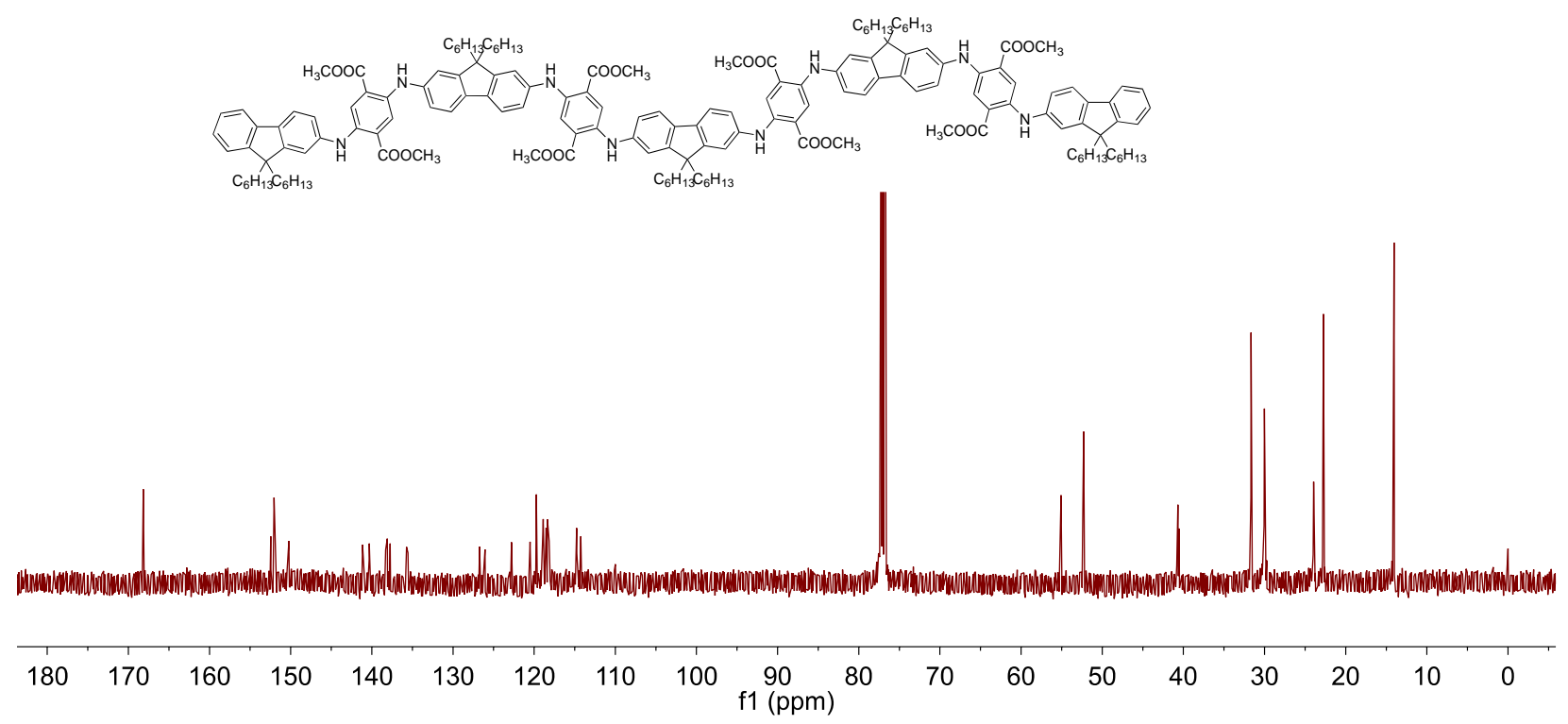

13C NMR of A4 (125 MHz, CDCl3, RT). 


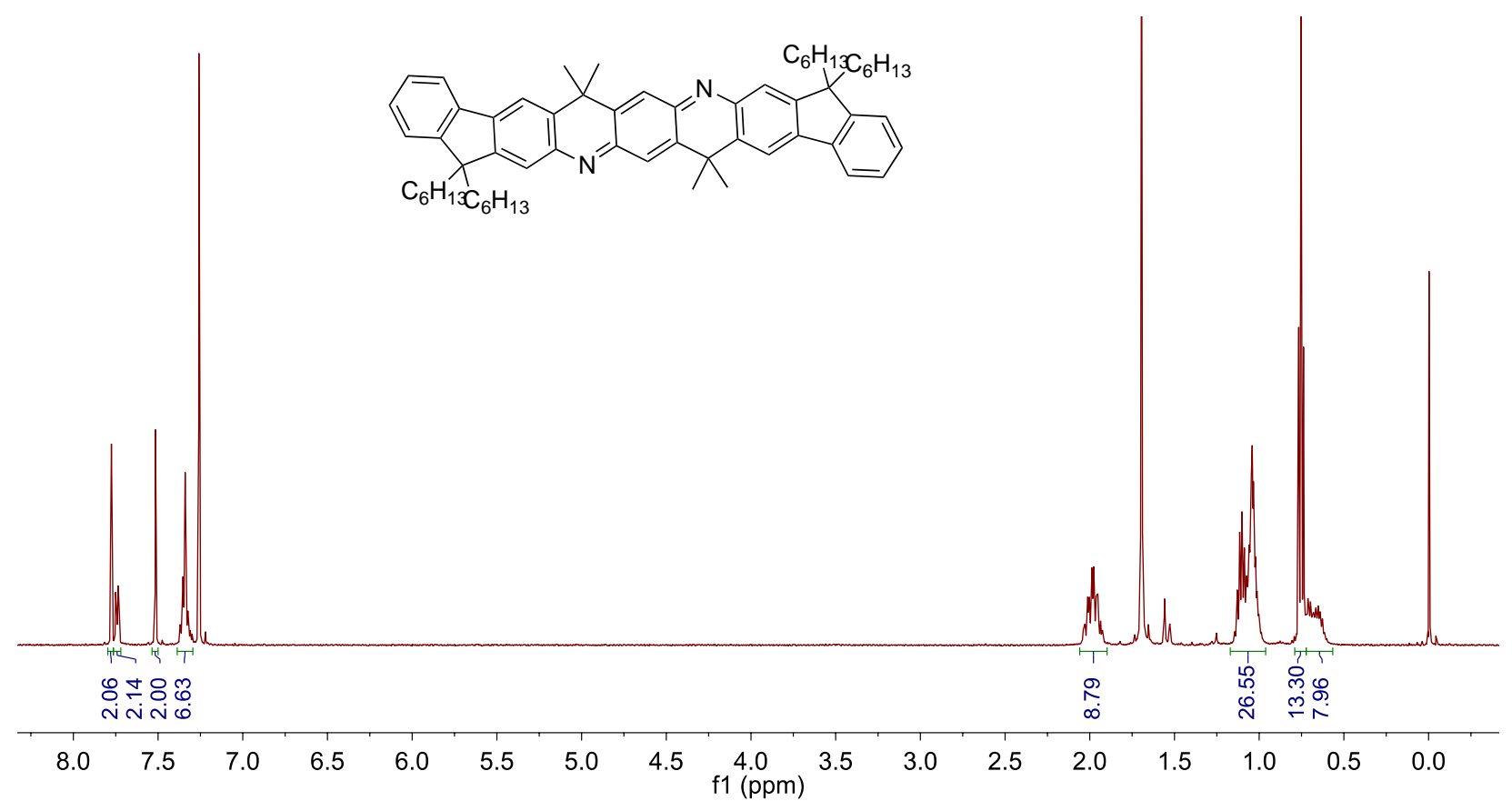

${ }_{1} \mathrm{H}$ NMR of Q1 (500 MHz, CDCl3, RT).
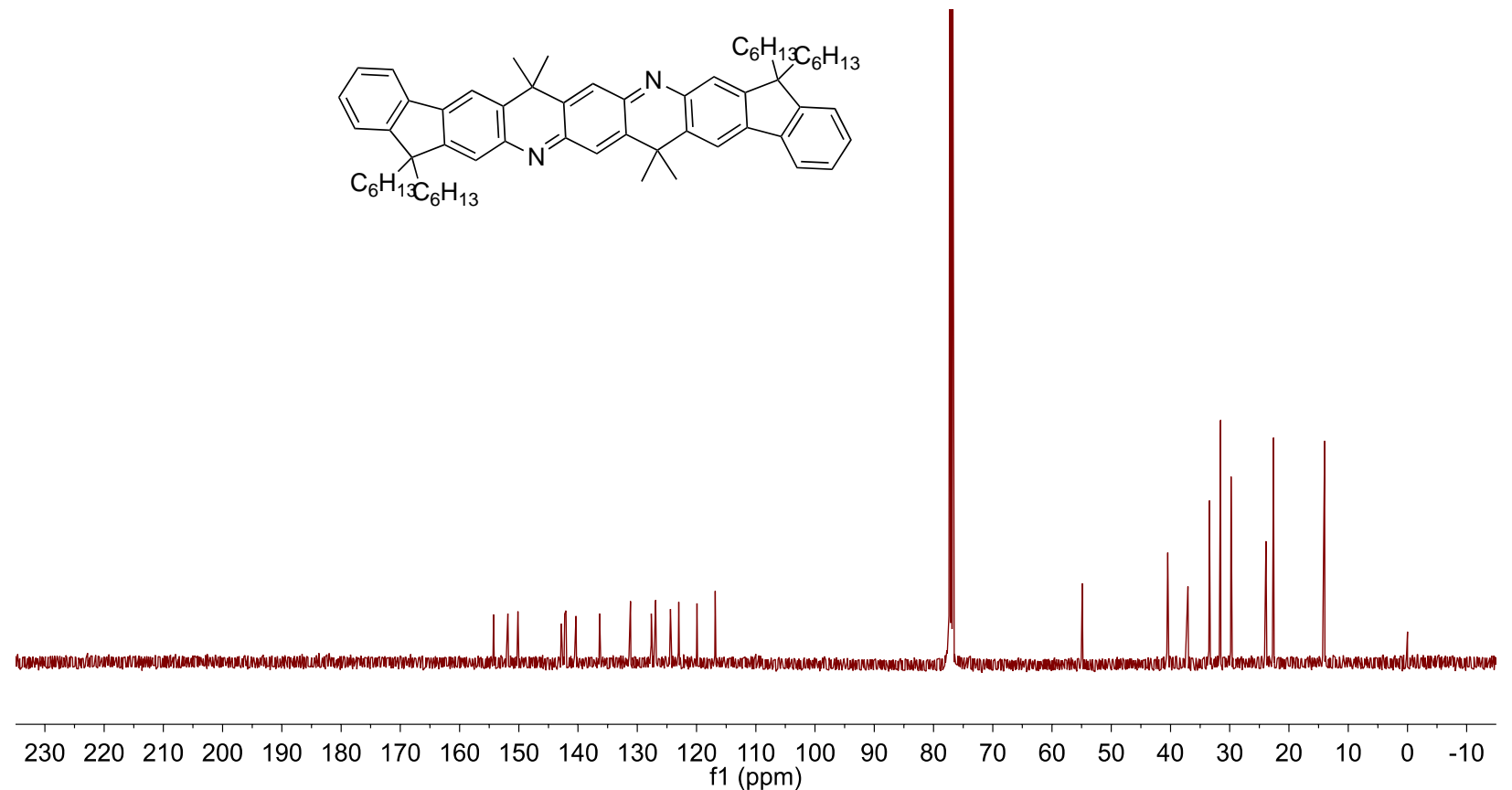

13C NMR of Q1 (125 MHz, CDCl3, RT). 


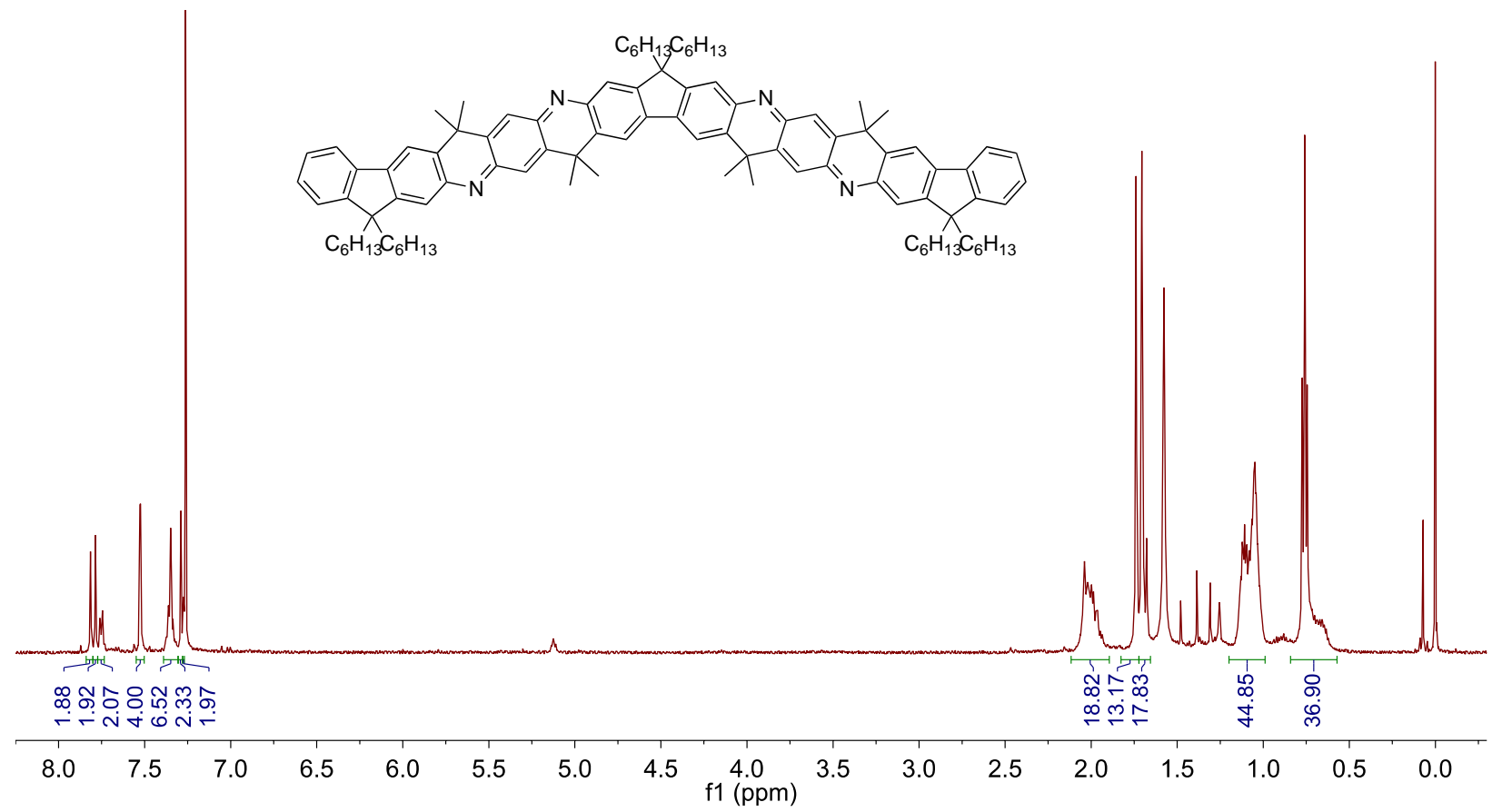

${ }_{1} \mathrm{H}$ NMR of Q2 (500 MHz, CDCl3, RT).

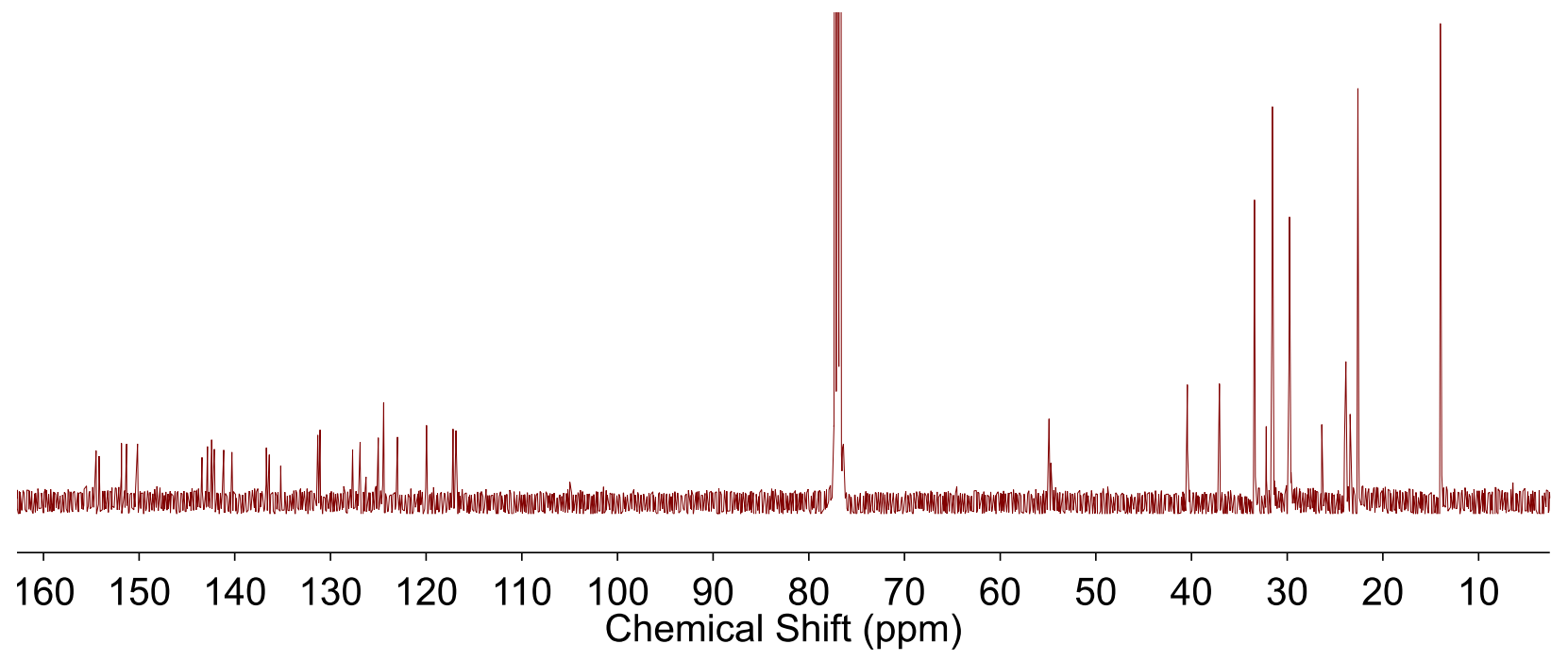
13C NMR of Q2 (125 MHz, CDCl3, RT). 


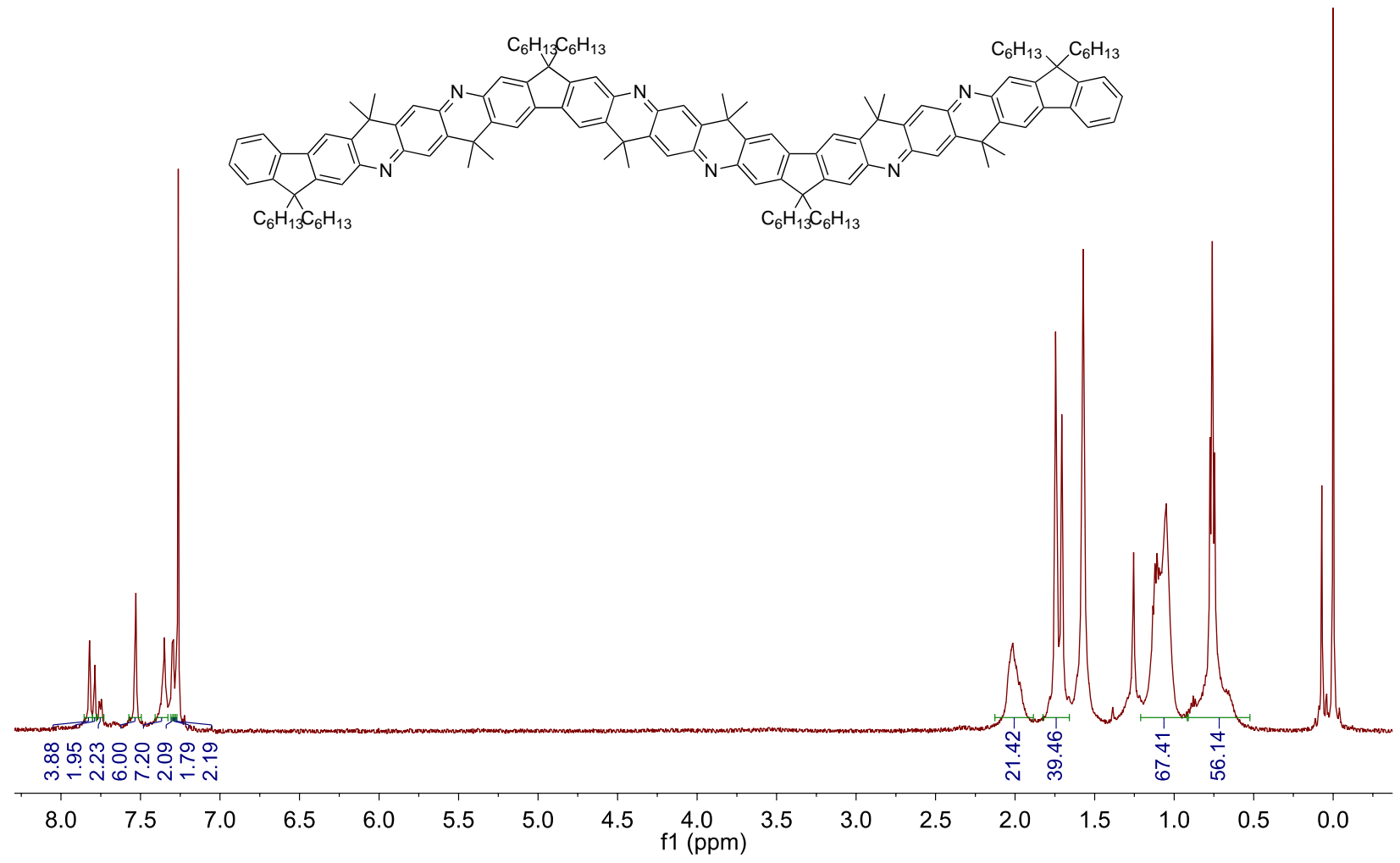

1H NMR of Q3 (500 MHz, CDCl3, RT).

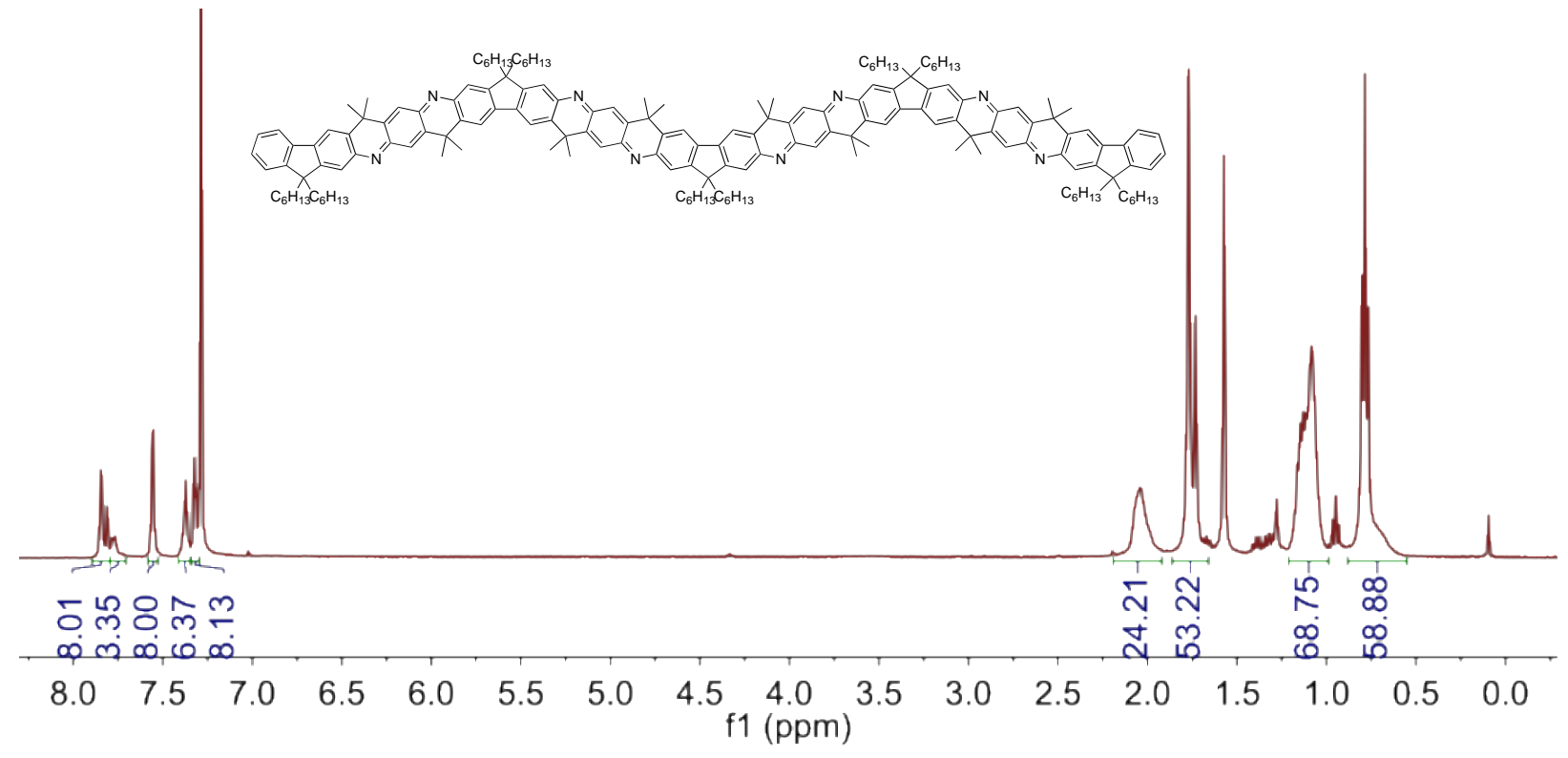

${ }_{1} \mathrm{H}$ NMR of Q4 (500 MHz, CDCl3, RT). 

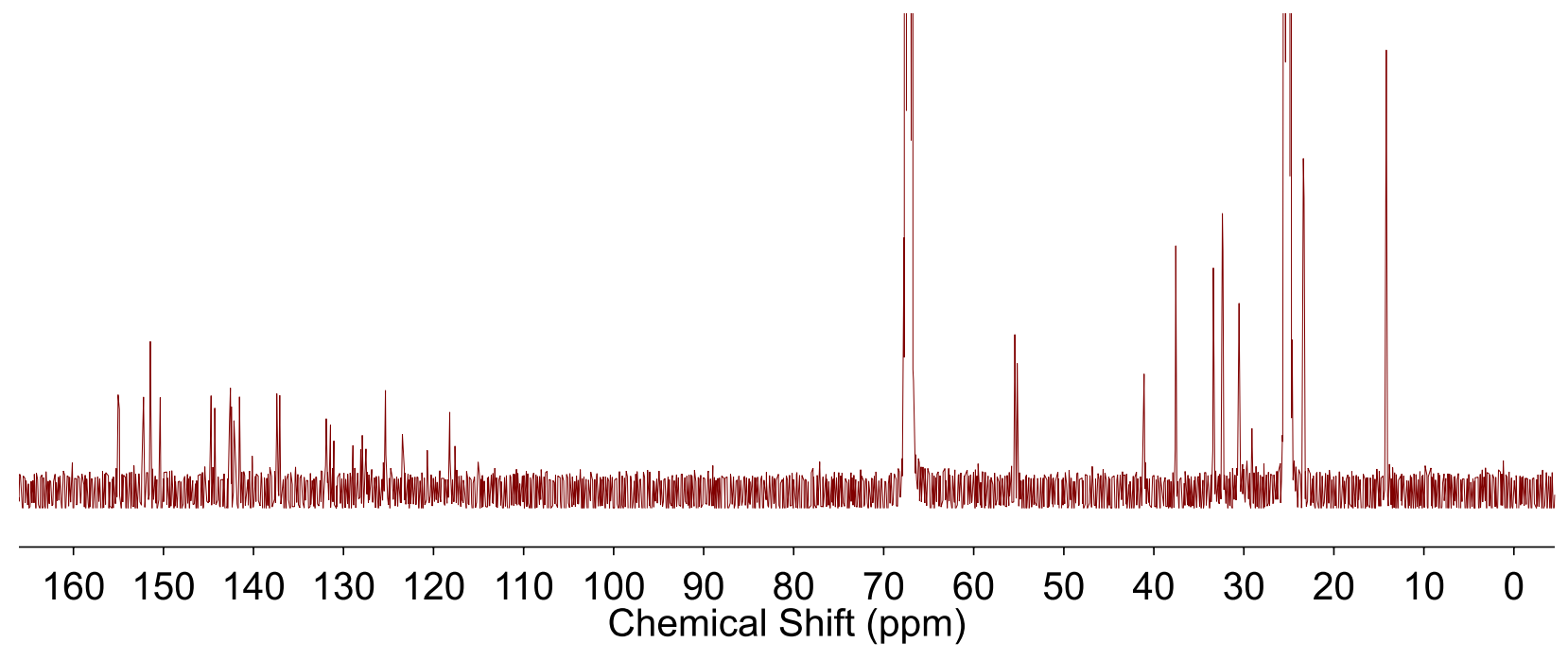

13C NMR of Q4 (125 MHz, d8-THF, RT).

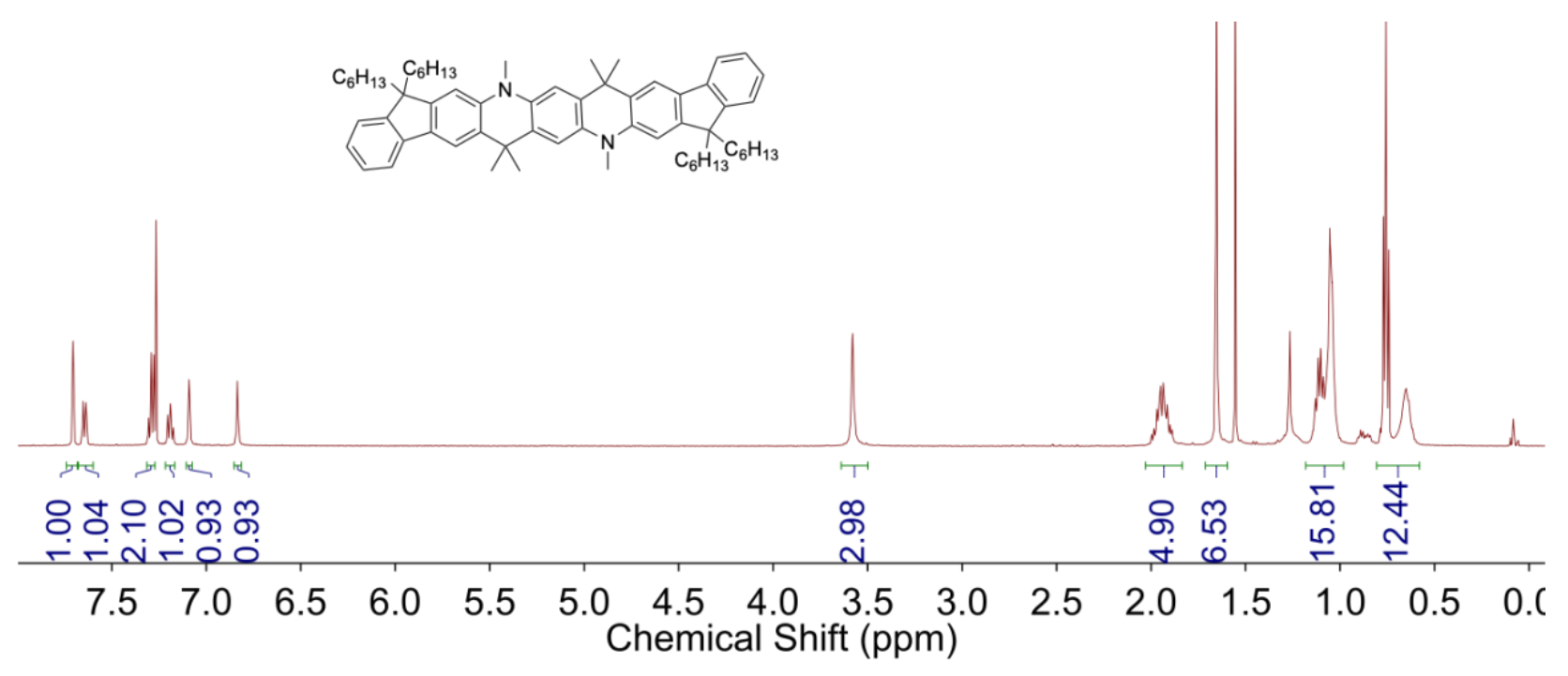

${ }_{1} \mathrm{H}$ NMR of Me-A (500 MHz, CDCl3, RT). 


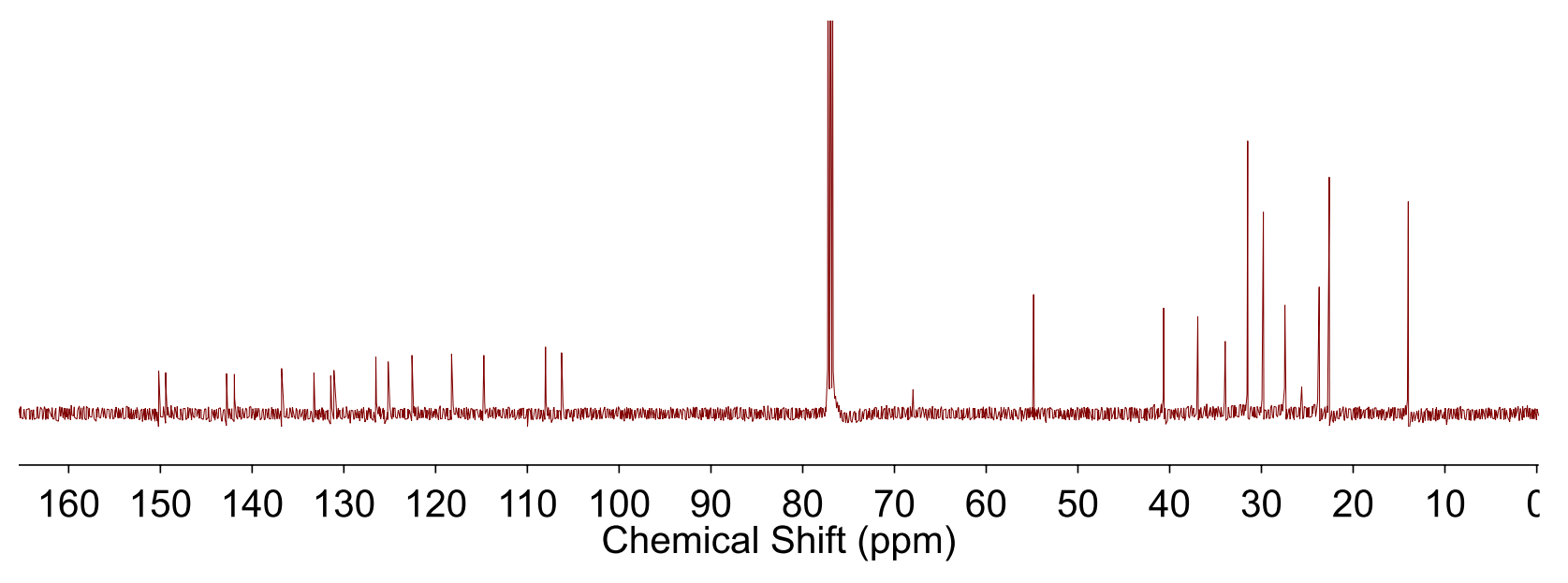

${ }_{13 \mathrm{C}} \mathrm{NMR}$ of Me-A (125 MHz, $\left.\mathrm{CDCl}_{3}, \mathrm{RT}\right)$.

\section{References}

1. Lungerich, D.; Papaianina, O.; Feofanov, M.; Liu, J.; Devarajulu, M.; Troyanov, S. I.; Maier, S.; Amsharov, K., Dehydrative $\pi$-extension to nanographenes with zig-zag edges. Nat. Commun. 2018, 9, 4756.

2. Beck, M.; Stiel, H.; Leupold, D.; Winter, B.; Pop, D.; Vogt, U.; Spitz, C., Evaluation of the energetic position of the lowest excited singlet state of beta-carotene by NEXAFS and photoemission spectroscopy. Bba-Bioenergetics 2001, 1506, 260-267.

3. Sandberg, M. O.; Nagao, O.; Wu, Z. K.; Matsushita, M. N.; Sugawara, T., Generation of a triplet diradical from a donor-acceptor cross conjugate upon acid-induced electron transfer. Chem Commun 2008, 3738-3740.

4. Rajca, A., Organic Diradicals and Polyradicals - from Spin Coupling to Magnetism. Chem Rev 1994, 94, 871-893.

5. Huggins, R. A., Simple method to determine electronic and ionic components of the conductivity in mixed conductors a review. Ionics 2002, 8, 300-313.

6. Hui, D.; Alexandrescu, R.; Chipara, M.; Morjan, I.; Aldica, G.; Chipara, M.; Lau, K., Impedance spectroscopy studies on doped polyanilines. Journal of Optoelectronics and Advanced Materials 2004, 6, 817-824.

7. Babu, V. J.; Vempati, S.; Ramakrishna, S., Conducting polyaniline-electrical charge transportation. Mater. Sci. Appl. 2013, 4, 1. 